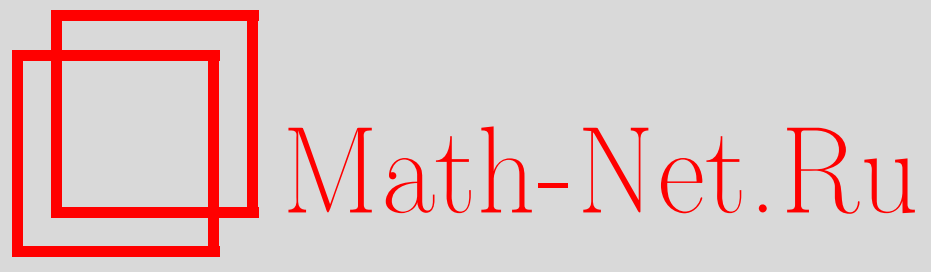

И. Х. Сабитов, Алгебраические методы решения многогранников, УМН, 2011, том 66, выпуск 3, 3-66

DOI: https://doi.org/10.4213/rm9396

Использование Общероссийского математического портала Math-Net.Ru подразумевает, что вы прочитали и согласны с пользовательским соглашением http://www . mathnet.ru/rus/agreement

Параметры загрузки:

IP: 52.205 .19 .152

26 апреля 2023 г., 09:35:46

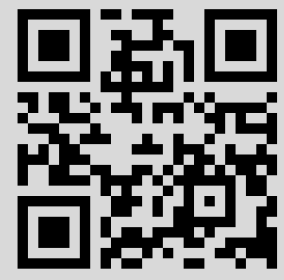




\title{
Алгебраические методы решения многогранников
}

\author{
И. Х. Сабитов
}

Решением многогранников, по аналогии с термином "решение треугольников", мы называем теорию и методы вычисления одних геометрических параметров многогранников через другие их параметры. В данной статье основное внимание уделяется обзору работ по вычислению объемов многогранников через их метрику и комбинаторное строение. Оказывается, что для объемов многогранников существует далеко идущее обобщение формулы Герона для площади треугольника, позволяющее доказать гипотезу о постоянстве объема изгибаемого многогранника в ходе его деформации.

Библиография: 110 названий.

Ключевые слова: многогранники, комбинаторное строение, метрика, объем, изгибание, гипотеза кузнечных мехов, многочлены объема, обобщение формулы Герона.

\section{СОДЕРЖАНИЕ}

1. Немного истории . ...................................... 4

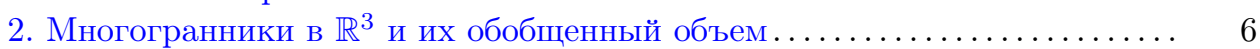

3. Мотивации для выбора пути решения задачи .................. 8

4. Основная теорема и ее следствия ........................... 11

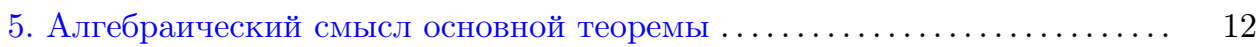

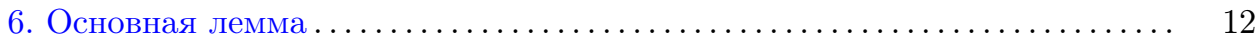

7. Определитель Кэли-Менгера................................ 13

8. Идея доказательства основной леммы ........................ 14

9. Доказательство основной теоремы . . . . . . . . . . . . . . . . . . . 18

10. Другое доказательство основной теоремы ..................... 19

11. Некоторые выводы . . . . . . . . . . . . . . . . . . . . . . . . . 23

12. Канонические многочлены объема ......................... 24

13. Вычисление многочлена объема для некоторых многогранников ..... 28

14. Вычисление длин диагоналей многогранников ................ 32

15. Обобщение уравнений для объема и диагоналей................... 39

16. Многогранники с известными диагоналями................... 40

17. Проблема изометрического погружения многогранной метрики...... 41

Работа частично поддержана грантами РФФИ № 09-01-00179 и № 10-01-91000АНФ. 
18. Некоторые алгебро-геометрические свойства многочленов объема .... . 43

18.1. Корни многочлена как объемы многогранника............. 44

18.2. Корни многочлена объема для изгибаемого многогранника.... 45

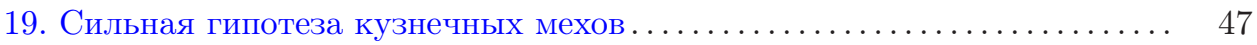

20. Еще один инвариант изгибаемых многогранников ............. 48

21. Многогранники в сферическом и гиперболическом пространствах ... 52

22. Многогранники в многомерных пространствах .............. 55

23. Некоторые нерешенные проблемы . . . . . . . . . . . . . . . . . . 57

Список литературы ...................................... 61

\section{1. Немного истории}

Решением многогранников, по аналогии с термином "решение треугольников", мы называем недавно появившееся направление в метрической теории многогранников, в котором изучается возможность вычисления одних характеристик многогранника (объем, длины ребер и диагоналей, двугранные углы и т.д.) через другие его метрические характеристики с учетом, конечно, его комбинаторного строения. Пока, в основном, известными характеристиками считаются метрика многогранника и его комбинаторное строение, и на основании их знания решаются такие вопросы, как существование многогранника с данной метрикой и данным комбинаторным строением, вычисление его объема, длин диагоналей, установление его изгибаемости или неизгибаемости, нахождение существующих изометрических деформаций и т. д.

Исторически первым в этом перечне был вопрос о единственности многогранника с данной метрикой и данным комбинаторным строением, изучение которого начинается с Лежандра. Он в первом издании своего известного учебника [1], анализируя в добавлении к нему (замечание XII) евклидово определение равенства многогранников ${ }^{1}$, приходит к выводу, что, во-первых, это определение должно рассматриваться на самом деле как теорема, требующая доказательства, и, во-вторых, нужно заранее предположить, что речь идет о выпуклых многогранниках, иначе определение 10 противоречит примеру, приведенному ранее английским математиком Симсоном (R. Simson, 1756 г.): куб с достроенной на одной его предварительно удаленной грани 4-угольной пирамидой вершиной наружу куба и другой равный куб с такой же пирамидой вершиной внутрь куба имеют "равные по количеству и величине" грани, но они, очевидно, не равны. Далее в том же замечании XII Лежандр для некоторых классов многогранников доказал, что два выпуклых многогранника с одинаковым комбинаторным строением и равными соответствующими гранями получаются один из другого движением, и высказал предположение, что такое же утверждение верно для любых выпуклых многогранников. По неизвестным нам причинам Лежандр в последующих изданиях (с 2-го по 9-е) своей книги убрал эту часть замечания XII и вернулся к ней только после того, как Коши в 1813 г. получил доказательство равенства действительно для всех выпуклых

\footnotetext{
1Это определение дано в ХІ книге "Начал" Евклида (мы приводим его по книге [2]):

ОпредЕление 10. Равные же и подобные телесные фигуры будут заключенные между равными по количеству и по величине подобными плоскостями.
} 
многогранников [3], причем в его доказательстве существенно использованы идеи Лежандра. Поэтому исторически правильно было бы эту теорему Коши называть теоремой Лежандра-Коши, как мы дальше и будем делать. ${ }^{2}$

Теорема Лежандра-Коши одновременно дает и утверждение о неизгибаемости (строго) выпуклых многогранников, так как в ходе изгибания (т. е. непрерывной деформации многогранника в предположении абсолютной твердости граней) многогранник в начальные моменты деформации остается выпуклым, следовательно, равным, по теореме Лежандра-Коши, исходному своему положению, иначе говоря, изгибание сводится только к движению многогранника как твердого тела.

После получения теоремы Лежандра-Коши естественным образом появился вопрос: верно ли утверждение о неизгибаемости для любых многогранников без предположения их выпуклости или же существуют изгибаемые многогранники? (Утверждение о равенстве в классе всех, в том числе и невыпуклых, многогранников опровергается примером Симсона.) Этот вопрос долгое время оставался открытым, пока в конце XIX века, в 1897 г., бельгийский математик и инженер Р. Брикар не доказал в работе [7] существование изгибаемых октаэдров, дав одновременно и их полную классификацию. Однако все эти изгибаемые октаэдры располагаются в пространстве $\mathbb{R}^{3}$, не будучи вложенными или даже погруженными, поэтому ответа на вопрос о существовании "настоящих" (т. е. без самопересечений) изгибаемых многогранников все равно не было. Более того, в 1975 г. Г. Глак доказал [8], что почти все гомеоморфные сфере многогранники являются неизгибаемыми и поэтому шансы на существование вложенных изгибаемых многогранников стали считаться ничтожными. Тем не менее американскому математику Роберту Коннелли удалось построить примеры изгибаемых многогранников, сначала погруженных [9], 1976 г., а затем и вложенных [10], 1977 г. Впоследствии были построены новые примеры изгибаемых многогранников (подробности можно найти в [11]) и у всех них было обнаружено, что в процессе изгибания их объем не изменяется. Это наблюдение дало основание выдвинуть гипотезу, что свойство инвариантности объема является общим для всех изгибаемых многогранников, см. [12] (февраль 1978 г., без упоминания автора гипотезы) и [13] (август 1978 г., со ссылкой на Д. Сулливана), но до сих пор, однако, не удалось установить, кто в действительности был первым ее автором ${ }^{3}$. Учитывая известный из физики закон $p V=$ Const, где $p$ - давление, a $V$ - объем газа, Коннелли [13] назвал эту гипотезу гипотезой "кузнечных мехов": математически идеально изготовленный изгибаемый многогранник (конечно, с отверстием, но расположенным на одной грани) не может быть использован в качестве кузнечных мехов.

Эта красивая гипотеза была, по-видимому, предметом размышлений многих геометров в течение почти двух десятилетий, но это осталось их личной тайной, так как в литературе были только две публикации [16] и [17], автор которых, новосибирский математик В. А. Александров, открыто писал, что его конечной целью было подтверждение или опровержение этой гипотезы. Кроме

\footnotetext{
${ }^{2}$ Есть работы, специально посвященные подробному анализу определения 10 и его связи с теоремой Лежандра-Коши (см., например, [4]-[6]).

ЗЭто неизвестно и авторам работ [14] и [15].
} 
того, в [18] приводятся примеры легко деформируемых многогранников с изменением их объема в ходе деформации, и можно предположить, что они тоже строились в расчете на опровержение гипотезы (но эти деформации не оказались изометрией). Наконец, в 1996 г. в работе [19] (первое продвижение в [20], более подробные доказательства в [21] и [22]) было доказано, что гипотеза справедлива для изгибаемых многогранников любого топологического рода.

Метод, использованный для ее доказательства, оказался очень полезным и в других вопросах метрической теории многогранников, и целью настоящей работы является краткое изложение доказательства гипотезы кузнечных мехов $^{4}$ и обзор других результатов, полученных в этой области геометрии в последние 10-15 лет с постановкой многих нерешенных задач. Наиболее близкими к теме данной статьи являются обзоры [24], [12] и [25]; популярное обсуждение этих вопросов можно найти в брошюре автора [11], а в [26] дано изложение похожих вопросов для плоских многоугольников вместе с большим списком нерешенных задач. Читателям, интересующимся более широким кругом вопросов метрической геометрии многогранников и многоугольников, можно рекомендовать большую работу [15] (насколько нам известно, она планируется к изданию как книга, но в настоящее время она доступна в виде препринта). Мы оставляем в стороне многочисленные работы, относящиеся к вопросам изгибаний и бесконечно малых изгибаний многогранников.

\section{2. Многогранники в $\mathbb{R}^{3}$ и их обобщенный объем}

Начнем с определений основных понятий, связанных с многогранниками в трехмерном пространстве. Всякий многогранник в трехмерном евклидовом пространстве $\mathbb{R}^{3}$ характеризуется своим комбинаторным строением, или комбинаторной схемой, и геометрической реализацией этой схемы в пространстве. Мы будем рассматривать многогранники только с треугольными гранями, поэтому за основу комбинаторной модели многогранника берем комбинаторную схему некоторого двумерного симплициального комплекса $K$. Многогранник с комбинаторным строением $K$, или геометрическую реализацию симплициального комплекса $K$ в $\mathbb{R}^{3}$, можно получить двумя способами.

Пусть $K$ сначала задан как двумерный абстрактный симплициальный комплекс с $n$ нульмерными симплексами, совокупность которых обозначим как некоторое множество $A=\left\{a_{1}, \ldots, a_{n}\right\}$.

ОПРЕДЕЛЕНИЕ 1а. Любое отображение $P: A \rightarrow \mathbb{R}^{3}$ называется многогранником в $\mathbb{R}^{3}$ с комбинаторным строением $K$, при этом:

1) образы нульмерных симплексов комплекса $K$ называются вериинами многогранника $P$;

2) одномерные симплексы, реализованные как прямолинейные отрезки, соединяющие образы соответствующих им пар нульмерных симплексов, называются ребрами многогранника;

3) гранями многогранника называются плоские треугольники, естественным образом соответствующие по отображению $P$ двумерным симплексам комплекса $K$.

\footnotetext{
${ }^{4}$ Второе доказательство гипотезы дано в [23] и далее оно тоже будет изложено.
} 
Второй способ определения многогранника предполагает, что $K$ задан как геометрический симплициальный комплекс, т. е. его симплексы заданы как точки, отрезки и двумерные плоские треугольники с указанием соответствующего закона отождествления точек и отрезков. Пусть $|K|$ обозначает тело комплекса $K$, понимаемое как теоретико-множественное объединение геометрических симплексов из $K$ с учетом закона их отождествления (иногда $|K|$ называют полиэдром комплекса $K$ ) и с соответствующей топологией.

ОПРеДЕЛЕНиЕ 1b. Многогранником в $\mathbb{R}^{3}$ с комбинаторным строением $K$ называется всякое отображение $P:|K| \rightarrow \mathbb{R}^{3}$, непрерывное в целом и линейное на каждом симплексе, а образ $P(|K|)$ называется геометрическим многогранником с комбинаторным строением $K$.

Так как линейное отображение любого симплекса полностью определяется значением отображения в его нульмерных симплексах, то очевидно, что оба определения многогранника эквивалентны. При работе с многогранниками на компьютере предпочтительно иметь дело с первым определением (в котором будущие вершины, ребра и грани многогранника задаются соответствующими списками), а второе определение более приспособлено для определения по аналогии многогранников с нетреугольными гранями как образами клеток.

Чтобы не уточнять каждый раз, каким способом - первым или вторым задано отображение, определяющее рассматриваемый многогранник, мы будем обозначать его всегда как $P: K \rightarrow \mathbb{R}^{3}$, и из этой записи видно, что речь идет о многограннике в $\mathbb{R}^{3}$ с комбинаторным строением $K$.

Оба (равносильных) определения многогранника $P$ таковы, что его геометрический образ в $\mathbb{R}^{3}$ может иметь самые разные особенности - вырождения ребер и граней, самопересечения и т. д., поэтому для такой многогранной поверхности нужно дать специальное определение понятия объема "ограниченной" ею пространственной области. Для этого мы в общем определении многогранника дополнительно предположим, что исходный геометрически заданный симплициальный комплекс $K$ гомеоморфен некоторому ориентируемому многообразию $M$ топологического рода $g \geqslant 0$ и он тогда задает некоторую триангуляцию многообразия $M$. Чтобы не вводить новых обозначений, будем считать, что $K$ уже сам представлен как триангулированное многообразие с прямолинейными симплексами в триангуляции (это то, что в топологической литературе обычно называют полиэдралъным или кусочно линейным многообразием). Пусть на $K$ задана некоторая ориентация. Перенесем эту ориентацию на все грани $P(K)$, указав на них соответствующий порядок обхода ребер.

ОПРЕДЕЛЕНИЕ 2. Обобщенным оббемом $V(P)$ ориентированного геометрического многогранника $P(K)$ называется сумма алгебраических объемов согласованно ориентированных тетраэдров с общей вершиной в некоторой точке $O \in \mathbb{R}^{3}$ и с основаниями на соответственно ориентированных гранях геометрического многогранника $P(K)$.

Напомним, что если вершины ориентированной грани $G \subset P(K)$ выписаны по нужному порядку обхода, скажем, в виде $p_{i}, p_{j}, p_{k}$, то алгебраический объем $V(T)$ ориентированного тетраэдра $T$ с вершиной в точке $O$ и с основанием на грани $G$ вычисляется как одна шестая смешанного произведения векторов, 
идущих от $O$ к вершинам грани $G$ и взятых в порядке следования вершин при обходе грани согласно ее ориентации. Отсюда легко вывести, что значение обобщенного объема многогранника не зависит от выбора точки $O$ и что для вложенного в $\mathbb{R}^{3}$ многогранника его обобщенный объем совпадает с обычным ориентированным объемом ${ }^{5}$.

Если в $\mathbb{R}^{3}$ введена некоторая система декартовых координат с началом в точке $O$, то, взяв в определении обобщенного объема в качестве точки $O$ начало координат, получаем следующее очевидное, но важное заключение: обобщенный обгем многогранника является однородным многочленом порядка 3 от координат вершин многогранника.

\section{3. Мотивации для выбора пути решения задачи}

Пусть вершины многогранника $P$ с $n$ вершинами пронумерованы в каком-либо порядке, и пусть $\left(x_{i}, y_{i}, z_{i}\right)$ - их координаты, $1 \leqslant i \leqslant n$. Тогда этому многограннику можно сопоставить точку $M\left(x_{1}, y_{1}, z_{1}, \ldots, x_{n}, y_{n}, z_{n}\right)$ пространства $\mathbb{R}^{3 n}$; обратно, каждой точке $M \in \mathbb{R}^{3 n}$ очевидным образом можно сопоставить точки $M_{i}\left(x_{i}, y_{i}, z_{i}\right), 1 \leqslant i \leqslant n$, пространства $\mathbb{R}^{3}$ и по ним, зная комбинаторную структуру $K$, построить соответствующий многогранник $P: K \rightarrow \mathbb{R}^{3}$. Следовательно, между множеством многогранников данного комбинаторного типа с $n$ вершинами и множеством точек пространства $\mathbb{R}^{3 n}$ существует взаимно однозначное соответствие $P \leftrightarrow M$, которое в соответствующей топологии можно считать гомеоморфизмом.

Пусть $e$ - число ребер комплекса $K$, и пусть ребра пронумерованы так, что каждой паре вершин $i$ и $j$, соединенных ребром, соответствует ребро с некоторым номером $k=k(i, j)=k(j, i), 1 \leqslant k \leqslant e$. Пусть $l_{k}$ - длина соответствующего ребра многогранника $P: K \rightarrow \mathbb{R}^{3}$. Тогда имеем уравнения

$$
\left(x_{i}-x_{j}\right)^{2}+\left(y_{i}-y_{j}\right)^{2}+\left(z_{i}-z_{j}\right)^{2}=l_{k}^{2}, \quad 1 \leqslant k=k(i, j) \leqslant e .
$$

При данных фиксированных длинах ребер $l_{k}$ все решения системы $(1)$ определяют, по отображению $M \rightarrow P$, множество всех многогранников в $\mathbb{R}^{3}$, имеющих одинаковое комбинаторное строение и изометричных между собой. Среди этих многогранников есть такие, которые получаются друг из друга движениями в $\mathbb{R}^{3}$ как твердых тел. Чтобы исключить такие "паразитические" решения, добавим к системе (1) уравнения

$$
\sum_{i} x_{i}=0, \quad \sum_{i} y_{i}=0, \quad \sum_{i} z_{i}=0,
$$

соответствующие приведению движением в $\mathbb{R}^{3}$ положения центра тяжести системы вершин многогранника к началу координат и исключающие дальнейший

\footnotetext{
${ }^{5}$ Если в многограннике $P(K)$ есть грань, вырожденная в отрезок или даже в точку, то ее ребрам мы приписываем ориентацию, унаследованную от ориентации соответствующего одномерного симплекса на прообразе грани в $K$. Объемы тетраэдров с основаниями на вырожденных гранях равны нулю независимо от ориентации основания, поэтому уточнение ориентаций таких граней нужно только для формально правильной записи соответствующего смешанного произведения для объема, чтобы в алгебраическом выражении суммы объемов получилась независимость от выбора общей вершины тетраэдров.
} 
параллельный перенос многогранника. Вращения исключить сложнее. Дело в том, что не существует уравнений (по крайней мере, нам они неизвестны), пригодных к исключению вращения всех многогранников данного комбинаторного типа во всех случаях, независимо от конфигурации многогранника и значений длин его ребер ${ }^{6}$. Например, уравнения, соответствующие приведению эллипсоида инерции системы вершин многогранника к главным осям,

$$
\sum_{i} x_{i} y_{i}=0, \quad \sum_{i} y_{i} z_{i}=0, \quad \sum_{i} x_{i} z_{i}=0,
$$

исключают вращение, толъко если $\sum_{i} x_{i}^{2} \neq \sum_{i} y_{i}^{2} \neq \sum_{i} z_{i}^{2}$ (т. е. когда эллипсоид инерции множества вершин многогранника не является эллипсоидом вращения). Вместо введения уравнений (3) можно считать, что одна грань многогранника занимает определенное фиксированное положение, исключающее возможность поворота многогранника, например, если есть грань $\langle 1,2,3\rangle$ и она невырождена, то к системе (1), (2) можем добавить три уравнения

$$
y_{2}=y_{1}, \quad z_{1}=z_{2}=z_{3},
$$

но эти уравнения не исключают вращения, если выбранная грань окажется вырожденной при некотором наборе значений длин ребер, т. е. мы не можем выбрать одну конкретную грань, фиксация положения которой устраняет вращение многогранника при всех допустимых (по неравенству треугольника) значениях длин ребер ${ }^{7}$. Тем не менее, при данных длинах ребер, предполагая не все грани метрического комплекса $K$ вырожденными, мы можем считать, что у нас, так или иначе, есть три уравнения, запрещающие непрерывные вращения многогранника.

Напомним, что множество многогранников в $\mathbb{R}^{3}$, имеющих одинаковое комбинаторное строение и изометричных между собой, называется конфигурационным пространством любого из этих многогранников. Учитывая интерпретацию каждого многогранника в $\mathbb{R}^{3}$ как точки в $\mathbb{R}^{3 n}$, получаем, что конфигурационное пространство любого данного многогранника $P$ в $\mathbb{R}^{3}$ гомеоморфно вещественному алгебраическому многообразию $\mathbf{A}$ в $\mathbb{R}^{3 n}$, определяемому системой (1), (2) с добавлением уравнений (3) или какого-либо их аналога. Уже для системы $(1),(2)$ легко показать, что при фиксированных значениях длин ребер все ее решения $\left(x_{1}, \ldots, z_{n}\right)$ расположены в $\mathbb{R}^{3 n}$ в шаре $B_{r}: x_{1}^{2}+\cdots+z_{n}^{2}<r^{2}$, где радиус $r=r(K, l)$ зависит от комбинаторного строения $K$ и метрики $l$ многогранника. Значит, многообразие А является компактным. Так как алгебраическое многообразие может иметь лишь конечное число компонент связности,

\footnotetext{
${ }^{6}$ Если речь идет об исключении непрерывных вращений одного данного многогранника, то такие уравнения известны (см. [12]).

7 Таким образом, задачу исключения непрерывных вращений можно формулировать как задачу замены абстрактной операции факторизации множества решений системы (1) (по признаку наложимости их друг на друга непрерывным вращением) добавлением трех уравнений, как это сделано для факторизации по параллельному переносу добавлением трех уравнений (2); иначе говоря, нужно уметь находить какую-либо алгебраическую поверхность, пересекающую в конечном числе точек каждую траекторию решений системы (1), получаемую под действием на них группы вращения пространства; см. об этом подробнее в [27; Problem Section].
} 
то конфигурационное пространство любого многогранника состоит из конечного числа компактных связных компонент, каждая из которых соответствует многогранникам, наложимым друг на друга некоторым нетривиальным изгибанием. Из них нульмерные (одноточечные) компоненты соответствуют неизгибаемым многогранникам, а компоненты размерности один и выше состоят из точек, соответствующих изометричным многогранникам, получаемым друг из друга изгибанием. Значит, если гипотеза кузнечных мехов верна, то многогранники из одной компоненты связности в $\mathbf{A}$ имеют одно и то же значение объема, а так как компонент конечное число, то отсюда заключаем, что если гипотеза кузнечных мехов верна, то множество значений объемов всех многогранников, имеющих данное комбинаторное строение и данный набор длин ребер, необходимо должно быть конечным. И обратно, если множество значений объема изометричных между собой многогранников конечно, то гипотеза кузнечных мехов верна. Это наблюдение было отмечено еще в 1989 г. в работе автора [28; с. 263].

Теперь встает вопрос: откуда можно заключить, что множество значений объема изометричных многогранников с одинаковым комбинаторным строением конечно? Естественно, сначала надо было исследовать простейшие случаи многогранники с малым количеством вершин. Рассмотрим случаи тетраэдра и 4-угольной пирамиды.

Обгем тетраэдра. Напомним, что если у тетраэдра (рис. 1) длины ребер равны $l_{1}, l_{2}, l_{3}, l_{4}, l_{5}, l_{6}$, то для его объема $V$ верна формула $^{8}$

$$
\begin{aligned}
V^{2}= & \frac{1}{144}\left[l_{1}^{2} l_{5}^{2}\left(l_{2}^{2}+l_{3}^{2}+l_{4}^{2}+l_{6}^{2}-l_{1}^{2}-l_{5}^{2}\right)+l_{2}^{2} l_{6}^{2}\left(l_{1}^{2}+l_{3}^{2}+l_{4}^{2}+l_{5}^{2}-l_{2}^{2}-l_{6}^{2}\right)\right. \\
& \left.+l_{3}^{2} l_{4}^{2}\left(l_{1}^{2}+l_{2}^{2}+l_{5}^{2}+l_{6}^{2}-l_{3}^{2}-l_{4}^{2}\right)-l_{1}^{2} l_{2}^{2} l_{3}^{2}-l_{2}^{2} l_{4}^{2} l_{5}^{2}-l_{1}^{2} l_{4}^{2} l_{6}^{2}-l_{3}^{2} l_{5}^{2} l_{6}^{2}\right] .
\end{aligned}
$$

Обобщенный обгем $V$ четырехугольной пирамиды (рис. 2) с триангулированным основанием можно вычислить как алгебраическую сумму объема $V_{1}$

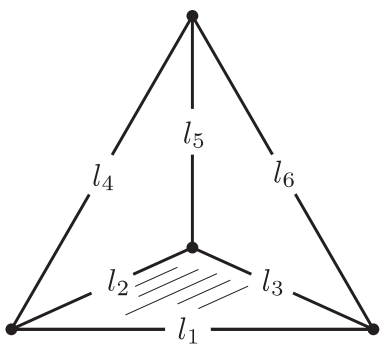

Рис. 1

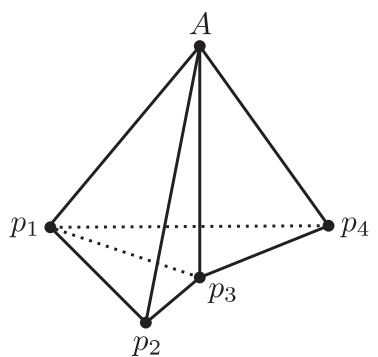

Рис. 2

тетраэдра $\left\langle A p_{1} p_{2} p_{3}\right\rangle$ и объема $V_{2}$ тетраэдра $\left\langle A p_{1} p_{3} p_{4}\right\rangle: V=V_{1} \pm V_{2} \Rightarrow V^{4}-$ $2\left(V_{1}^{2}+V_{2}^{2}\right) V^{2}+\left(V_{1}^{2}-V_{2}^{2}\right)^{2}=0$, и так как $V_{1}^{2}$ и $V_{2}^{2}$ выражаются по предыдущей

${ }^{8} \mathrm{~K}$ сожалению, в наших работах [21; формула (1)] и [25; формула (3)] для объема тетраэдра в четырех последних слагаемых есть ошибка в индексах ребер, которая здесь исправлена (в каждом из четырех слагаемых должны быть индексы трех сторон, ограничивающих грани). 
формуле (4) через квадраты длин соответствующих ребер, то для обобщенного объема $V$ четырехугольной пирамиды имеем полиномиальное уравнение вида

$$
Q(V)=V^{4}+a_{1}(l) V^{2}+a_{2}(l)=0 .
$$

Таким образом, мы приходим к выводу, что в простейших случаях объем многогранника является корнем некоторого многочлена со старшим коэффициентом 1, а остальные его коэффициенты являются в свою очередь многочленами от квадратов длин ребер многогранника с числовыми коэффициентами, которые определяются комбинаторным строением многогранника. Значит, можно было ожидать, что желанная конечность множества значений обгемов многогранников с данной метрикой и данным комбинаторным строением следует из того, что обгем является корнем некоторого многочлена, не зависящего от конкретной конфигурации многогранника в пространстве. Это предположение было впервые высказано в написанном автором $\S 8$ работы [29].

\section{4. Основная теорема и ее следствия}

Оказывается, ожидаемая ситуация действительно имеет место и верна следующая основная теорема.

Теорема 1. Пусть $[P]$ - множество всех многогранников в $\mathbb{R}^{3}$ с треугольными гранями, имеющих один и тот же комбинаторный тип $K$ и одни и те же длины соответствующих ребер $\left(l_{1}, \ldots, l_{e}\right)$, где е - число ребер. Тогда существует некоторый приведенный ${ }^{9}$ многочлен

$$
Q(l, V)=V^{2 N}+a_{1}(l) V^{2 N-2}+\cdots+a_{N-1}(l) V^{2}+a_{N}(l)
$$

такой, что обобщенный облем каждого многогранника из $[P]$ является корнем этого многочлена, в котором коэффициенты $a_{i}(l), 1 \leqslant i \leqslant N$, сами являются полиномами от квадратов длин ребер многогранника с числовыми коэффициентами, зависящими от комбинаторного строения многогранника ${ }^{10}$.

Из этой теоремы вытекают два очевидных следствия.

СлеДСТвИЕ 1. Множество значений обобщенных оббемов всех изометричных между собой многогранников с одинаковым комбинаторным строением конечно.

СЛЕДСТВИЕ 2. Обобщенный объем изгибаемого многогранника в ходе изгибания остается постоянным.

Действительно, объем в ходе изгибания многогранника должен изменяться непрерывно, а непрерывная функция, принимающая конечное число значений, должна быть постоянной. Таким образом, утверждение гипотезы кузнечных мехов оказалось простым следствием основной теоремы, которую естественно интерпретировать как обобщение формуль Герона на многогранники.

\footnotetext{
${ }^{9}$ Так мы называем многочлены со старшим коэффициентом 1 ; для таких многочленов есть и другие названия, например, в некоторых работах их называют нормированными или мономиальными.

10 Через $l$ обозначен набор квадратов длин ребер многогранника.
} 


\section{5. Алгебраический смысл основной теоремы}

Как было отмечено в самом конце раздела 2, объем $V$ многогранника представляется как однородный многочлен степени 3 от координат вершин многогранника; это мы запишем в виде соотношения $V=V(x)$, понимая под $x$ совокупность координат $x_{1}, y_{1}, z_{1}, \ldots, x_{n}, y_{n}, z_{n}$ всех $n$ вершин многогранника. Далее, уравнения (1) выражают зависимость квадратов длин ребер многогранника тоже от координат вершин; это мы запишем как $l=l(x)$, понимая под $l$ весь набор квадратов длин ребер. Тогда тот факт, что основная теорема верна при любой конфигурации и при любом расположении многогранника в пространстве, отражается в том, что при подстановке в многочлен $Q(V)$ вместо $V$ и $l$ их выражений через координаты вершин многогранника мы получаем тождественное равенство $Q(l(x), V(x)) \equiv 0$ относительно переменных $x_{1}, y_{1}, z_{1}, \ldots, x_{n}, y_{n}, z_{n}$. Это значит, что между многочленами 2-й степени от $x$, дающими значения квадратов длин ребер, и многочленом 3-й степени для объема существует алгебраическая зависимость. Поэтому любой приведенный многочлен от переменных $l_{1}^{2}, \ldots, l_{e}^{2}, V^{2}$, обращающийся в тождественный нуль при подстановке вместо $V^{2}$ и $l_{i}^{2}, 1 \leqslant i \leqslant e$, их выражений через координаты вершин многогранника данного комбинаторного типа $K$, мы далее будем называть многочленом объема для многогранников данного комбинаторного строения $K$.

\section{6. Основная лемма}

Для формулировки основной леммы нам надо условиться о некоторой терминологии.

ОПРЕДЕЛЕНИЕ 3. Если известно, что для некоторого многогранника верна теорема 1 , то будем говорить, что этот многогранник обладает свойством $Q$.

ОПРЕДЕЛЕНИЕ 4. Если трехзвенный цикл, образованный тремя ребрами многогранника, не является границей никакой грани, то такой цикл будем называть пустым.

Примером пустого цикла является цикл $\left\langle A p_{1} p_{3}\right\rangle$ на рис. 2.

ОПРЕДЕЛЕНИЕ 5. Будем говорить, что многогранник имеет комбинаторное cmpoение muna $K_{0}$, если в нем есть хотя бы одна вершина, не инцидентная никакому пустому циклу, т. е. через которую не проходит ни один пустой цикл (такие вершины будем называть для краткости “хорошими" вершинами).

Примерами многогранников с комбинаторным строением типа $K_{0}$ являются тетраэдр и октаэдр, в которых каждая вершина является “хорошей". Многогранник на рис. 2 тоже комбинаторного типа $K_{0}$, так как в нем вместе с "нехорошими" есть и "хорошие" вершины. Контрпримером является многогранник Часара [30] типа тора, имеющий 7 вершин - этот многогранник не имеет диагоналей и на границе звезды любой его вершины каждая пара вершин соединена ребром, так что через каждую вершину многогранника проходит 9 пустых циклов (триангуляцию и рисунок такого многогранника см., например, в [31]). Можно показать, что в любом многограннике рода $g=0$ и с числом вершин 
больше 4 есть по крайней мере две "хорошие" вершины, причем не соединенные ребром [32].

Отметим, что если в многограннике есть вершина степени 3, то такой многогранник имеет комбинаторный тип $K_{0}$.

Теперь мы можем сформулировать основную лемму.

ЛЕмма 1. Если все многогранники фиксированного топологического рода $g \geqslant 0$ и с $n$ вершинами обладают свойством $Q$, то многогранники того же топологического рода и комбинаторного типа $K_{0} c n+1$ вершинами тоже обладают свойством $Q$.

\section{7. Определитель Кэли-Менгера}

В серии работ 50-60-х годов 19-го века Кэли изучал геометрию расположения точек на плоскости и, в частности, рассмотрел вопрос о том, когда 6 положительных чисел могут быть попарными расстояниями между 4 точками на плоскости. Затем, в 20-е годы 20-го века, аналогичный вопрос в случае общей размерности $n$ был изучен Менгером. Решение этого вопроса подробно

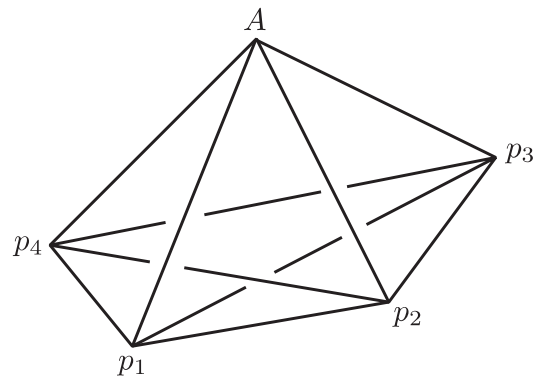

Рис. 3

рассмотрено в книге [33], мы приведем оттуда лишь одно нужное нам утверждение: 10 расстояний между 5 точками $A, p_{1}, p_{2}, p_{3}, p_{4}$ в $\mathbb{R}^{3}$ (рис. 3) необходимо удовлетворяют уравнению Кэли-Менгера

$$
\operatorname{det}(C M)=\left|\begin{array}{cccccc}
0 & 1 & 1 & 1 & 1 & 1 \\
1 & 0 & l_{A 1}^{2} & l_{A 2}^{2} & l_{A 3}^{2} & l_{A 4}^{2} \\
1 & l_{1 A}^{2} & 0 & l_{12}^{2} & l_{13}^{2} & l_{14}^{2} \\
1 & l_{2 A}^{2} & l_{21}^{2} & 0 & l_{23}^{2} & l_{24}^{2} \\
1 & l_{3 A}^{2} & l_{31}^{2} & l_{32}^{2} & 0 & l_{34}^{2} \\
1 & l_{4 A}^{2} & l_{41}^{2} & l_{42}^{2} & l_{43}^{2} & 0
\end{array}\right|=0
$$

(через $l_{A i}=l_{i A}, 1 \leqslant i \leqslant 4$, обозначено расстояние между точками $A$ и $p_{i}$, a $l_{i j}$ обозначает расстояние между точками $p_{i}$ и $p_{j}$ ). Геометрический смысл уравнения Кэли-Менгера следующий: объем $V_{T}$ тетраэдра с 5 вершинами в $\mathbb{R}^{4}$ 
выражается формулой $V_{T}^{2}=-\frac{1}{2^{4}(4 !)^{2}} \operatorname{det}(C M)$ и уравнение Кэли-Менгера получается из условия, что 5 вершин 4-мерного тетраэдра будут находиться в $\mathbb{R}^{3}$ только в том случае, когда его 4-мерный объем равен нулю.

Нам нужно будет использовать уравнение Кэли-Менгера в ситуации, когда известны не все расстояния, поэтому некоторые из них мы обозначим специальным образом. Именно, пусть $x=l_{13}^{2}, y=l_{24}^{2}$. Тогда большими, но простыми вычислениями получаем равенство вида

$$
\begin{aligned}
-8 \operatorname{det}(C M)=x^{2}\left[y^{2}+a_{1}(l) y+b_{1}(l)\right] & \\
& +x\left[a_{2}(l) y^{2}+b_{2}(l) y+b_{3}(l)\right]+b_{4}(l) y^{2}+b_{5}(l) y+b_{6}(l)=0,
\end{aligned}
$$

в котором коэффициенты $a_{1}(l)$ и $a_{2}(l)$ не обращаются в нуль, если нет трех точек, лежащих на одной прямой.

\section{8. Идея доказательства основной леммы}

Для доказательства леммы 1 мы предполагаем, что многогранник $P$ с $n+1$ вершинами, имеющий комбинаторный тип $K_{0}$, находится в общем положении: координаты его вершин алгебраически независимы, т. е. они не удовлетворяют никакому алгебраическому уравнению. Доказательство проводится индукцией по степени имеющихся "хороших" вершин (напомним, что степенью вершины называется число исходящих из нее ребер). Пусть в $P$ есть вершина $A$ степени 3. Удалим открытую звезду этой вершины, получится многогранник с треугольным краем. Заклеив этот край треугольной гранью, получим многогранник $P_{1}$ с $n$ вершинами. При этой операции мы не добавили ни одного нового ребра, так что все ребра многогранника $P_{1}$ имеют известные длины. По предположению индукции многогранник $P_{1}$ обладает свойством $Q$, поэтому для его объема $V_{1}\left(P_{1}\right)$ имеем полиномиальное уравнение вида

$$
Q_{1}\left(V_{1}\right)=V_{1}^{2 N_{1}}+a_{1}^{(1)} V_{1}^{2 N_{1}-2}+\cdots+a_{N_{1}-1}^{(1)} V_{1}^{2}+a_{N_{1}}^{(1)}=0,
$$

в котором коэффициенты $a_{i}^{(1)}$ зависят от квадратов длин ребер многогранника $P_{1}$, т. е. от квадратов длин ребер многогранника $P$, за исключением трех исходящих из вершины $A$ ребер. Объемы $V$ и $V_{1}$ соответственно многогранников $P$ и $P_{1}$ связаны равенством $V_{1}=V+\varepsilon V_{T}$, где $V_{T}$ - объем тетраэдра $T$ с вершиной $A$, а $\varepsilon= \pm 1$ (так как объем удаляемого тетраэдра должен или прибавляться, или вычитаться в зависимости от положения вершины $A$ ). Подставляя в уравнение $Q_{1}\left(V_{1}\right)=0$ значение $V_{1}=V+\varepsilon V_{T}$ и учитывая, что квадрат объема тетраэдра $T$ по формуле (4) явным образом выражается через квадраты длин его ребер, получаем, что объем $V^{2}(P)$ является корнем уравнения вида $Q(V)=V^{4 N_{1}}+a_{1}(l) V^{4 N_{1}-2}+\cdots=0$.

Далее используем индукцию по степени имеющейся "хорошей" вершины. Разберем подробно случай, когда есть “хорошая” вершина степени 4.

Итак, пусть $A$ - некоторая "хорошая" вершина степени 4, не инцидентная никакому пустому 3 -циклу. Обозначим через $\operatorname{St} A$ звезду вершины $A$. Перенумеруем вершины края St $A$, начав с произвольной вершины, в некотором 
циклическом порядке как $p_{1}, p_{2}, p_{3}, p_{4}$. На основе $P$ мы теперь построим два новых многогранника $P_{1}$ и $P_{2}$, имеющих вершину степени 3 . Именно, для построения $P_{i}$ мы удаляем ребро $\left\langle A p_{i}\right\rangle, i=1,2$, с двумя прилегающими гранями $\left\langle p_{i-1} A p_{i}\right\rangle,\left\langle p_{i} A p_{i+1}\right\rangle$ (считаем, что $p_{0}=p_{4}$ ) и заменяем их новыми гранями $\left\langle p_{i-1} p_{i} p_{i+1}\right\rangle$ и $\left\langle p_{i-1} A p_{i+1}\right\rangle$ (см. рис. 4). Эти построения возможны, так как диагонали $\left\langle p_{1} p_{3}\right\rangle$ и $\left\langle p_{2} p_{4}\right\rangle$ не являются ребрами многогранника $P$ и поэтому в новых многогранниках $P_{1}$ и $P_{2}$ каждая из них инцидентна точно двум новым добавленным граням. В многогранниках $P_{1}$ и $P_{2}$ вершина $A$ имеет степень 3 .
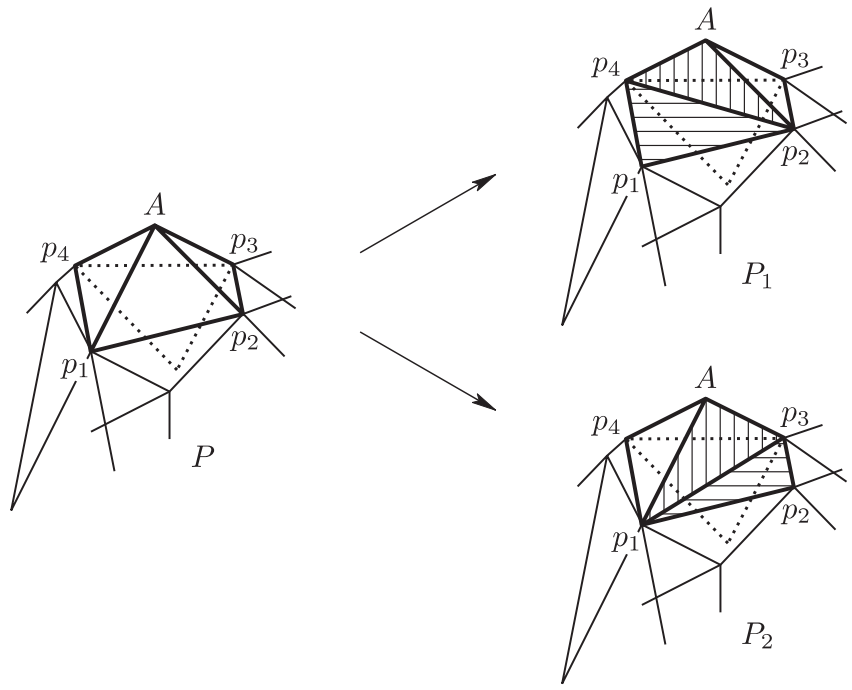

Рис. 4

Обозначим длины диагоналей $\left\langle p_{4} p_{2}\right\rangle$ и $\left\langle p_{1} p_{3}\right\rangle$ соответственно $D_{1}$ и $D_{2}$ (так что нижний индекс указывает номер точки, находящейся "между" концами диагонали по ходу обхода 4-угольника). По базе индукции объемы $V_{1}=\operatorname{vol}\left(P_{1}\right)$ и $V_{2}=\operatorname{vol}\left(P_{2}\right)$ являются корнями некоторых полиномиальных уравнений вида

$$
Q_{i}=V_{i}^{2 N_{i}}+a_{1}^{(i)}\left(l, D_{i}^{2}\right) V_{i}^{2 N_{i}-2}+\cdots=0, \quad i=1,2 .
$$

Пусть $V_{0 i}$ - объем тетраэдра $\left\langle A p_{i-1} p_{i} p_{i+1}\right\rangle$, дополняющего $P_{i}$ до $P$. Тогда для объема $V(P)$ мы имеем

$$
V_{i}=V-\varepsilon_{i} V_{0 i}, \quad \varepsilon_{i}= \pm 1, \quad i=1,2 .
$$

Подставляя значение $V_{i}$ из (10) в (9) и используя равенства

$$
V_{i}^{2 m}=\sum_{k=1}^{m-1} C_{2 m}^{2 k} V^{2 k} V_{0 i}^{2(n-k)}-\varepsilon_{i} V V_{0 i} \sum_{k=0}^{m-1} C_{2 m}^{2 k+1} V^{2 k} V_{0 i}^{2(m-k-1)},
$$

мы после очевидных преобразований получаем уравнения вида

$$
V^{4 N_{i}}+A_{1}^{(i)}\left(l, D_{i}^{2}, V_{0 i}^{2}\right) V^{4 N_{i}-2}+\cdots+A_{2 N_{i}}^{(i)}\left(l, D_{i}^{2}, V_{0 i}^{2}\right) V^{0}=0
$$


уже без $\varepsilon_{i}$. Это означает, что полученные уравнения не зависят от конкретной позиции St $A$ относительно оставшейся части $P$. Подставляя теперь в $(10)$ значение $V_{0 i}^{2}$ из (4) как функции от $\left(l, D_{i}^{2}\right)$, мы приходим к уравнениям

$$
V^{4 N_{i}}+B_{1}^{(i)}\left(l, D_{i}^{2}\right) V^{4 N_{i}-2}+\cdots+B_{2 N_{i}}^{(i)}\left(l, D_{i}^{2}\right) V^{0}=0, \quad i=1,2 .
$$

Мы можем рассматривать (12) как полиномиальные уравнения относительно $D_{i}^{2}$. Для этого представим (12) в виде

$$
C_{0}^{(i)}\left(l, V^{2}\right) D_{i}^{2 K_{i}}+\cdots+C_{K_{i}}^{(i)}\left(l, V^{2}\right)=0, \quad i=1,2,
$$

где через $2 K_{i} \leqslant 12 N_{i}$ обозначена наибольшая степень $D_{i}$ в полиномиальном уравнении (12), когда величины $l_{1}^{2}, \ldots, l_{e}^{2}$ с обозначением $l$ считаются независимыми переменными (или “буквенными величинами"). Если для одного из значений $i=1$ или $i=2$ имеем $K_{i}=0$, то соответствующее уравнение (13) принимает вид $C_{K_{i}}^{(i)}\left(l, V^{2}\right)=V^{4 N_{i}}+\cdots=0$. Тут возможны два варианта. Подставим в это последнее уравнение значения объема $V$ и квадратов длин сторон как функций от координат вершин. Если при этом получим тождественный нуль, то мы уже имеем для $V$ нужный многочлен вида (6): $Q(V)=C_{K_{i}}^{(i)}\left(l, V^{2}\right)=V^{4 N_{i}}+\cdots$. Если же получится многочлен, который не равен нулю тождественно, тогда окажется, что координаты вершин рассматриваемого многогранника связаны алгебраической зависимостью, что для многогранника в общем положении невозможно. Значит, или мы уже имеем искомый многочлен вида (6), или $K_{i}>0$. Пусть $K_{i}>0, i=1,2$. Подставим в $C_{j}^{(i)}\left(l, V^{2}\right), 0 \leqslant j<K_{i}$, значения $l=\left(l_{1}^{2}, \ldots, l_{e}^{2}\right)$ и $V$ как полиномиальных функций от $\left(x_{1}, \ldots, z_{n}\right)$. Если все полученные после этой операции функции будут тождественно равны нулю, то мы снова получаем искомое уравнение $Q(V)=C_{K_{i}}^{(i)}\left(l, V^{2}\right)=V^{4 N_{i}}+\cdots=0$. Если же по крайней мере одна из этих функций не обращается в тождественный нуль, то для рассматриваемого $P$ коэффициент при соответствующей степени $D_{i}$ вообще не равен нулю, так как $P$ по предположению находится в общем положении. Для краткости обозначений предположим, что первый такой коэффициент совпадает с $C_{0}^{(i)}\left(l, V^{2}\right)$, так что мы имеем уравнения $(12)$ с $C_{0}^{(i)}\left(l, V^{2}\right) \neq 0$.

Теперь вспомним, что 10 расстояний между 5 точками $A, p_{0}, p_{1}, p_{2}, p_{3}$ удовлетворяют уравнению Кэли-Менгера (8). В нашем случае $x=D_{1}^{2}, y=D_{2}^{2}$, а остальные расстояния известны - это длины соответствующих ребер $P$, так что мы можем использовать для них обозначение $l$. Тогда соответствующее уравнение Кэли-Менгера может быть записано в виде

$$
\bar{D}_{1}^{2}\left(\bar{D}_{2}^{2}+a \bar{D}_{2}+b\right)+\bar{D}_{1}\left(a \bar{D}_{2}^{2}+b \bar{D}_{2}+b\right)+\left(b \bar{D}_{2}^{2}+b \bar{D}_{2}+b\right)=0
$$

где $\bar{D}_{1}=D_{1}^{2}, \bar{D}_{2}=D_{2}^{2}$ (мы не пишем переменные $p$ и $q$, так как они в рассматриваемом случае известны - это длины ребер $\left\langle p_{0} p_{2}\right\rangle$ и $\left.\left\langle p_{0} p_{2}\right\rangle\right)$; кроме этого, для краткости записи мы не стали писать у коэффициентов индексы. Так как старшие коэффициенты в (12) при $i=1$ и в (14) отличны от нуля, мы можем исключить $\bar{D}_{1}$, используя для этого результант уравнений (13) и $(14)$, что нам 
дает уравнение

$\left|\begin{array}{cccccc}\left(\bar{D}_{2}^{2}+\cdots\right) & \left(a \bar{D}_{2}^{2}+\cdots\right) & \left(b \bar{D}_{2}^{2}+\cdots\right) & 0 & 0 & 0 \\ 0 & \left(\bar{D}_{2}^{2}+\cdots\right) & \left(a \bar{D}_{2}^{2}+\cdots\right) & 0 & 0 & 0 \\ \vdots & \vdots & \ddots & \vdots & \vdots & \vdots \\ 0 & 0 & \cdots & \left(\bar{D}_{2}^{2}+\cdots\right) & \left(a \bar{D}_{2}^{2}+\cdots\right) & \left(b \bar{D}_{2}^{2}+\cdots\right) \\ C_{0}^{(1)}\left(l, V^{2}\right) & C_{1}^{(1)}\left(l, V^{2}\right) & \cdots & * & \left(V^{4 N_{1}}+\cdots\right) & 0 \\ 0 & C_{0}^{(1)}\left(l, V^{2}\right) & \cdots & * & * * & \left(V^{4 N_{1}}+\cdots\right)\end{array}\right|=0$

(здесь $*$ и ** обозначают коэффициенты при $\bar{D}_{1}^{0}$ в уравнениях (14) и (13) при $i=1$. Напомним, что в коэффициентах $C_{j}^{(1)}, j<K_{1}$, степени $V$ меньше чем $4 N_{1}$, так что после раскрытия этого определителя размером $\left(K_{1}+2\right) \times\left(K_{1}+2\right)$ мы получаем уравнение вида

$$
\begin{aligned}
& \bar{D}_{2}^{2 K_{1}}\left(V^{8 N_{1}}+\cdots\right)+\bar{D}_{2}^{2 K_{1}-1}\left(a V^{8 N_{1}}+\cdots\right) \\
& \quad+\bar{D}_{2}^{2 K_{1}-2}\left(b V^{8 N_{1}}+\cdots\right)+\cdots+\bar{D}_{2}^{0}\left(b V^{8 N_{1}}+\cdots\right)=0 .
\end{aligned}
$$

Теперь мы исключаем $\bar{D}_{2}$ из уравнения (15) и уравнения (13) при $i=2$, переписанного для $\bar{D}_{2}=D_{2}^{2}$. Легко видеть, что соответствующий результант дает уравнение

$$
\left|\begin{array}{cccccc}
\left(V^{8 N_{1}}+\cdots\right) & \left(a V^{8 N_{1}}+\cdots\right) & \ldots & * & \ldots & 0 \\
0 & \left(V^{8 N_{1}}+\cdots\right) & \ldots & \ldots & \ldots & \ldots \\
\vdots & \ddots & \vdots & \vdots & \ldots & \ldots \\
0 & \ldots & \left(V^{8 N_{1}}+\cdots\right) & \ldots & \ldots & * \\
C_{0}^{(2)}\left(l, V^{2}\right) & C_{1}^{(2)}\left(l, V^{2}\right) & \ldots & \left(V^{4 N_{2}}+\cdots\right) & \ldots & 0 \\
\vdots & \ddots & \vdots & \ddots & \vdots & \\
0 & \ldots & C_{0}^{(2)}\left(l, V^{2}\right) & \ldots & \ldots & \left(V^{4 N_{2}}+\cdots\right)
\end{array}\right|=0 .
$$

Отсюда видим, что наибольшая степень при $V$ равна $2 N=8 K_{2} N_{1}+8 K_{1} N_{2}$ (так как степени $V$ в коэффициентах $C_{i}^{(2)}$ меньше чем $4 N_{2}$ ), причем старший коэффициент равен 1, т. е. мы получили уравнение искомого вида $Q(V)=$ $V^{2 N}+\cdots=0$, где степень $2 N$ многочлена $Q(V)$ допускает оценку $2 N \leqslant 96 N_{1} N_{2}$ (эта очень грубая оценка может быть существенно улучшена, но для доказательства леммы и соответственно теоремы это не имеет принципиального значения). Для случая, когда степень вершины $A$ равна 4 , шаг индукции сделан.

Обосновывать шаг индукции при рассмотрении “хорошей” вершины произвольной степени $m \geqslant 5$ мы здесь не будем, это сделано, например, в работах [19], [21], [22].

Для завершения доказательства леммы остается заметить следующее. Все наши рассуждения были справедливы в предположении, что рассматриваемый многогранник $P$ находится в общем положении. Если это не так, то мы приведем $P$ малым возмущением в общее положение и составим для близких многогранников $P_{\varepsilon}$ уравнения $Q_{\varepsilon}\left(V_{\varepsilon}\right)=0$ вида $(6)$, в которых функции-многочлены 
$a_{i}\left(l_{\varepsilon}\right)$ имеют некоторые числовые коэффициенты, не зависящие от $\varepsilon$ (зависящие только от комбинаторного строения $P$ и от выбора вершины $A$ ). Переходя к пределу при $\varepsilon \rightarrow 0$ и учитывая, что в коэффициентах многочленов $Q_{\varepsilon}$ длины $l_{\varepsilon}$ непрерывно зависят от $\varepsilon \rightarrow 0$, мы получаем, что $V(P)$ удовлетворяет тому же самому уравнению (6) с коэффициентами $a_{i}(l)$. Лемма 1 доказана.

ЗАмЕчаниЕ 1. Как видно из проведенных рассуждений, нахождение нужного многочлена $Q(V)$ дается конструктивным способом, но этот способ не определяется единственным образом, а зависит на каждом шаге от выбора "хорошей" вершины и от способа сведения вопроса к многограннику с "хорошей" вершиной меньшей степени.

\section{9. Доказательство основной теоремы 1}

Воспользуемся индукцией по числу вершин $n$ и по роду $g$, база индукции формула для объема тетраэдра. Пусть сначала род $g$ рассматриваемых многогранников равен нулю, и пусть теорема доказана для таких многогранников с числом вершин $n$. Пусть многогранник $P$ рода 0 имеет $n+1$ вершин. Можно показать, что в любом многограннике рода $g=0$ существует "хорошая" вершина (их даже по крайней мере 2, см. [32]), и поэтому все такие многогранники имеют комбинаторный тип $K_{0}$. А тогда применима основная лемма, и мы получим существование требуемого полиномиального уравнения $Q(l, V)=0 .{ }^{11}$

Пусть теорема доказана для всех многогранников рода $g$. Рассмотрим многогранники рода $g+1$. Пусть $P_{0}$ - многогранник рода $g+1$ с наименьшим числом вершин $n_{\min }$ (формулу для $n_{\min }$ в зависимости от значения рода $g$ можно найти в [34]). Тогда он обязательно имеет трехзвенный цикл, не ограничивающий грань. Действительно, возьмем в $P_{0}$ любую вершину $A$. Если через $A$ не проходит трехзвенный цикл требуемого вида, то можно убрать звезду вершины $A$ и заклеить полученное отверстие треугольными гранями, сторонами которых могут служить исходящие из одной вершины диагонали $\partial(\mathrm{St} A)$ (так как ни одна диагональ края звезды не является ребром). Тогда получим новый многогранник того же рода $g$, но с числом вершин меньше чем у $P_{0}$, что противоречит определению $P_{0}$. Значит, в $P_{0}$ есть трехзвенный цикл, не ограничивающий грань. Разрезав $P_{0}$ по этому циклу и заклеив образовавшиеся треугольные отверстия двумя гранями, получим или один, или два многогранника меньшего рода, а для них свойство $Q$ уже есть. Если многогранник $P_{0}$ после этой операции не распался (а это его свойство сохраняется для всех его изометричных реализаций, так как оно определяется только комбинаторным строением многогранника), то его объем будет среди корней многочлена $Q$ для полученного после заклеивания отверстия многогранника меньшего рода. Пусть получились два многогранника $P_{1}$ и $P_{2}$, оба меньшего рода. По индукционному предположению для каждого из них свойство $Q$ выполнено. Объем $V_{0}\left(P_{0}\right)$ связан с объемами $V_{1}\left(P_{1}\right)$ и $V_{2}\left(P_{2}\right)$ равенством

$$
V_{0}\left(P_{0}\right)=\varepsilon_{1} V_{1}\left(P_{1}\right)+\varepsilon_{2} V_{2}\left(P_{2}\right), \quad \varepsilon_{i}= \pm 1, \quad i=1,2 .
$$

\footnotetext{
${ }^{11}$ В [21] есть нужное рассуждение без использования существования “хорошей” вершины.
} 
Так как для $V_{1}$ и $V_{2}$ есть полиномиальные уравнения вида $Q_{1}\left(l, V_{1}\right)=0$ и $Q_{2}\left(l, V_{2}\right)=0$, в коэффициентах которых участвуют только длины ребер из $P_{0}$, то из этих уравнений и уравнения (16) легко исключить $V_{1}$ и $V_{2}$ и получить нужное уравнение вида $Q\left(l, V_{0}\right)=0$. Следовательно, для многогранников рода $g$ база индукции есть - мы доказали, что для них многогранник с наименьшим числом вершин обладает свойством $Q$. А дальше применяем индукцию с использованием основной леммы, а в случае невозможности ее применения (т. е. когда многогранник не комбинаторного типа $K_{0}$ ) разрезаем многогранник по имеющемуся трехзвенному циклу и сводим вопрос или к многограннику того же топологического рода, но с меньшим числом вершин, или же к многогранникам с большим числом вершин, но меньшего рода, для которых свойство $Q$ установлено. Теорема доказана.

\section{0. Другое доказательство основной теоремы 1}

Можно показать, что если для многогранников ввести величину $\widetilde{V}=12 \mathrm{~V}$, то мономиальное уравнение $Q(V)=V^{2 N}+a_{1}(l) V^{2 N-2}+\cdots=0$ с рациональными числовыми коэффициентами для многочленов $a_{i}(l)$ превратится в мономиальное уравнение

$$
\widetilde{Q}(\widetilde{V})=\widetilde{V}^{2 N}+\tilde{a}_{1}(l) \widetilde{V}^{2 N-2}+\cdots=0
$$

с целыми коэффициентами в многочленах $\tilde{a}_{i}(l)$, в которых переменными попрежнему являются квадраты длин ребер. Напомним, что если нам даны некоторое поле $L$ и кольцо $R \subset L$, то элемент $x \in L$ называется целым над кольцом $R$, если существуют натуральное число $N$ и элементы $a_{i} \in R, 1 \leqslant i \leqslant N$, такие, что $x$ удовлетворяет уравнению вида

$$
x^{N}+a_{1} x^{N-1}+\cdots+a_{N}=0 .
$$

Следовательно, алгебраический смысл уравнения (6), объясненный в разделе 6 и распространенный на уравнение (17), по-другому можно выразить утверждением, что если нам дан многогранник $P$ с известным комбинаторным строением и с известными координатами вершин, то элемент $\widetilde{V}$ поля $\mathbb{R}$ является целым над кольцом, порожденным квадратами длин ребер многогранника (это кольцо как раз и состоит из элементов, представимых как значения многочленов от квадратов длин ребер с целочисленными коэффициентами). А в алгебре известен критерий целостности элемента поля над данным подкольцом поля. Применение этого критерия дает второе доказательство основной теоремы, что и сделано в работе [23].

Опишем кратко идею этого доказательства, следуя в основном его изложению в [14], с некоторыми необходимыми уточнениями. Для этого напомним некоторые понятия из теории нормированиц ${ }^{12}$. Пусть $L$ и $F$ - некоторые поля. Нормированием на $L$ со значениями в $F$ называется отображение

\footnotetext{
12 Этим термином, по рекомендации Э. Б. Винберга, мы переводим английское "theory of places"; используемый в некоторых работах перевод-калька "теория плейсов", так же как буквальный перевод “теория мест", нам не кажутся подходящими для русскоязычной терминологии, а возможный вариант “теория положений”, хотя и близок по смыслу к оригинальному термину "place", кажется неприемлемым для алгебраистов, навевая уводящие в сторону геометрические ассоциации.
} 
$\varphi: L \rightarrow F \cup\{\infty\}$ такое, что для всех элементов $x, y \in L$ выполнены условия:
1) $\varphi(x+y)=\varphi(x)+\varphi(y)$,
2) $\varphi(x y)=\varphi(x) \varphi(y)$,
3) $\varphi(1)=1$.

Элементы из $F$ называются конечными, а в отношении действий с символом $\infty$ принимаются следующие соглашения: $\infty \cdot \infty=\infty$ и если $a \in F$, то $a \pm \infty=\infty$, $a / \infty=0$ и $a / 0=\infty, a \cdot \infty=\infty$, если $a \neq 0$. Выражения $\infty / \infty, \infty \pm \infty$ и $0 / 0$, $0 \cdot \infty$ не определены и вышеприведенные равенства 1$), 2)$ имеют смысл только при условии, что их правые части определены.

Нормирования на $L$ дают следующий критерий целостности элементов из $L$.

УтВеРЖДЕНИЕ 1. Пусть поле L содержит некоторое колъцо $R$. Тогда для того чтобы элемент $x \in L$ был целым над кольцом $R$, необходимо и достаточно, чтобы любое нормирование на $L$, конечное на элементах из $R$, было конечным и на $x$.

Доказательство этого критерия можно найти, например, в [35] или [36]. Из него мы получаем простое, но важное в дальнейших рассуждениях следствие.

СлеДствиЕ 3. Если элементы $x, y$ из $L$ - иелье над некоторым кольщом $R$, то $x+y$ u $x-y$ тоже целье над $R$.

Формулу (4) для объема $V$ тетраэдра можно представить в виде уравнения

$$
(12 V)^{2}+a_{1}\left(l_{1}^{2}, l_{2}^{2}, l_{3}^{2}, l_{4}^{2}, l_{5}^{2}, l_{6}^{2}\right)=0,
$$

где $a_{1}(l)$ - явно выписанный в (4) многочлен от квадратов длин ребер тетраэдра. Следовательно, по введенной выше терминологии, элемент $(12 V)^{2} \in \mathbb{R}$ является целым над кольцом, порожденным элементами $l_{1}^{2}, \ldots, l_{6}^{2}$. Далее доказательство будет проходить по индукции, как и в первом доказательстве, по числу вершин $n$ и по топологическому роду $g$ многогранника. Индукционный переход будет опираться на аналог основной леммы 1 , а его доказательство будет основано на следующей лемме.

Лемма 2. Пусть в $\mathbb{R}^{3}$ даны $n+1$ различных точек $q, p_{1}, p_{2}, \ldots, p_{n}, n \geqslant 4$, с алгебраически независимыми координатами $x_{0}, y_{0}, z_{0}, \ldots, z_{n}$. Обозначим через $l_{i}$ длины $\left|q p_{i}\right|$ и через $l_{i, j}-$ длинь $\left|p_{i} p_{j}\right|$, с отождествлением нумерации по модулю $п$. Пусть $\varphi$ - нормирование на поле $L=\mathbb{Q}\left(x_{0}, \ldots, z_{n}\right)$, состоящем из рациональных функиий от $x_{1}, \ldots, z_{n}$ с иельми коэфбициентами (включая функиию, тождественно равную единище) и содержсащем подкольцо $R$, порожденное квадратами указанных выше длин, рассматриваемыми как элементы поля $\mathbb{Q}$ по формуле (1). Предположим, что оно конечно на всех элеменmax $l_{i}^{2}$ u $l_{i, i+1}^{2}$. Тогда существует $i, 1 \leqslant i \leqslant n-2$, для которого $\varphi\left(l_{i, i+2}^{2}\right)$ конечно.

ДокАЗАТЕЛЬСТво ЛЕммы 2. Допустим противное:

$$
\varphi\left(l_{i, i+2}^{2}\right)=\infty, \quad 1 \leqslant i \leqslant n-2 .
$$

Покажем, что тогда для всех $i, 3 \leqslant i \leqslant n$, будут выполнены равенства

$$
\varphi\left(l_{1, i}^{2}\right)=\infty \quad \text { и } \quad \varphi\left(\frac{l_{1, i}^{2}}{l_{1, i-1}^{2}}\right)=\infty .
$$


Действительно, эти равенства верны для $i=3$ в силу условия леммы и по допущению противного. Если, предположив, что они справедливы для некоторого $i>3$, мы покажем, что они верны и для $i+1$, то последнее равенство $\varphi\left(l_{1, n}^{2}\right)=\infty$ будет противоречить условию леммы.

Пусть (20) верно для некоторого $i, i>3$. Покажем, что тогда

$$
\varphi\left(\frac{l_{1, i+1}^{2}}{l_{1, i}^{2} l_{i-1, i+1}^{2}}\right) \neq 0 .
$$

Снова допустим обратное:

$$
\varphi\left(\frac{l_{1, i+1}^{2}}{l_{1, i}^{2} l_{i-1, i+1}^{2}}\right)=0 .
$$

Выпишем для 10 расстояний между 5 точками $p_{1}, p_{i-1}, p_{i}, p_{i+1}, q$ уравнение Кэли-Менгера (7):

$$
\operatorname{det}(C M)=\left|\begin{array}{cccccc}
0 & 1 & 1 & 1 & 1 & 1 \\
1 & 0 & l_{1, i-1}^{2} & l_{1, i}^{2} & l_{1, i+1}^{2} & l_{1}^{2} \\
1 & l_{1, i-1}^{2} & 0 & l_{i-1, i}^{2} & l_{i-1, i+1}^{2} & l_{i-1}^{2} \\
1 & l_{1, i}^{2} & l_{i-1, i}^{2} & 0 & l_{i, i+1}^{2} & l_{i}^{2} \\
1 & l_{1, i+1}^{2} & l_{i-1, i+1}^{2} & l_{i, i+1}^{2} & 0 & l_{i+1}^{2} \\
1 & l_{1}^{2} & l_{i-1}^{2} & l_{i}^{2} & l_{i+1}^{2} & 0
\end{array}\right|=0 .
$$

Разделив элементы 2-й строки и 2-го столбца на $l_{1, i}^{2} \neq 0$, а элементы 5 -й строки и 5-го столбца - на $l_{i-1, i+1}^{2} \neq 0$, получим

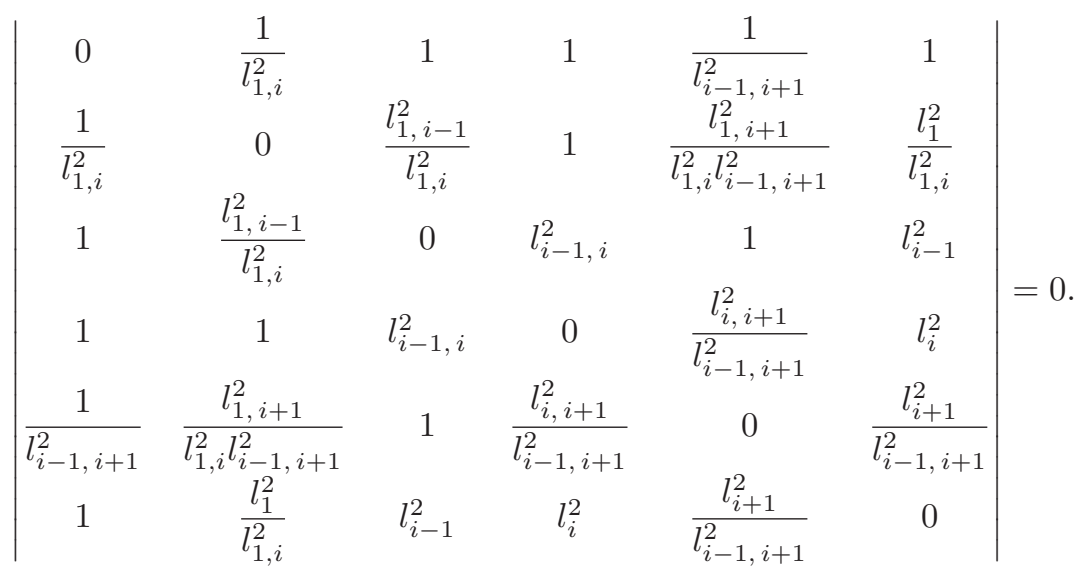

Применим к этому определителю операцию нормирования $\varphi$. Учитывая предположения (19), (21) и равенства (20) при $i>3$, получаем, что

$$
\varphi(0)=\left|\begin{array}{cccccc}
0 & 0 & 1 & 1 & 0 & 1 \\
0 & 0 & 0 & 1 & 0 & 0 \\
1 & 0 & 0 & \varphi\left(l_{i-1, i}^{2}\right) & 1 & \varphi\left(l_{i-1}^{2}\right) \\
1 & 1 & \varphi\left(l_{i-1, i}^{2}\right) & 0 & 0 & \varphi\left(l_{i}^{2}\right) \\
0 & 0 & 1 & 0 & 0 & 0 \\
1 & 0 & \varphi\left(l_{i-1}^{2}\right) & \varphi\left(l_{i}^{2}\right) & 0 & 0
\end{array}\right|=0 .
$$


Однако непосредственное вычисление последнего определителя дает значение $\varphi(0)=-1$, следовательно, предположение (21) неверно, т. е.

$$
\varphi\left(\frac{l_{1, i+1}^{2}}{l_{1, i}^{2} l_{i-1, i+1}^{2}}\right) \neq 0 .
$$

Тогда

$$
\varphi\left(\frac{l_{1, i+1}^{2}}{l_{1, i}^{2} l_{i-1, i+1}^{2}}\right)=\frac{\varphi\left(l_{1, i+1}^{2} / l_{1, i}^{2}\right)}{\varphi\left(l_{i-1, i+1}^{2}\right)} \neq 0 .
$$

В последнем неравенстве выражение $\varphi\left(l_{i-1, i+1}^{2}\right)$ в знаменателе, по предположению (19), равно $\infty$, следовательно, выражение $\varphi\left(l_{1, i+1}^{2} / l_{1, i}^{2}\right)$ в числителе не может быть конечным, что и показывает справедливость индукционного перехода в доказательстве соотношений (20). Но тогда, как было сказано, при $i=n$ приходим к противоречию с условием леммы. Следовательно, невозможность предположения (19), а вместе с этим и справедливость леммы 2 доказана.

Теперь мы можем доказать аналог основной леммы 1, примененной в первом доказательстве.

Лемма 3. Если у симплициального многогранника $P$ существует "хорошая" вершина, то проверку иелостности его обгема над колъцом, порожденным квадратами длин его ребер, можно свести к проверке челостности оббема некоторого многогранника с "хорошей" вершиной менъшей степени.

ДокАЗАТЕЛЬСтво. Сначала предположим, что многогранник $P$ находится в общем положении, т. е. координаты $x_{1}, \ldots, z_{n}$ всех $n$ вершин многогранника алгебраически независимы. Пусть $\varphi$ - некоторое нормирование на поле $\mathbb{Q}\left(x_{1}, \ldots, z_{n}\right)$, конечное над элементами кольца $R$, порожденного квадратами длин ребер многогранника $P$ с объемом $V$. Пусть $q$ - "хорошая" вершина степени $m$ многогранника $P$. Рассмотрим звезду $T$ этой вершины. Пусть $p_{1}, p_{2}, \ldots, p_{m}-$ пронумерованные в циклическом порядке вершины края $T$. По лемме 2 существуют точки $p_{i_{0}-1}$ и $p_{i_{0}+1}$, для которых значение $\varphi\left(l_{i_{0}-1, i_{0}+1}^{2}\right)$ конечно. Удалим в многограннике $P$ две грани $\left\langle q p_{i_{0}-1} p_{i_{0}}\right\rangle$ и $\left\langle q p_{i_{0}} p_{i_{0}+1}\right\rangle$ вместе с ребром $q p_{i_{0}}$, затем закроем образовавшееся 4-угольное отверстие двумя плоскими треугольниками $q p_{i_{0}-1} p_{i_{0}+1}$ и $p_{i_{0}-1} p_{i_{0}} p_{i_{0}+1}$. Получится новый симплициальный многогранник $P^{\prime}$ с теми же вершинами, но у которого вершина $q$, по-прежнему являясь "хорошей", имеет на единицу меньшую степень. Пусть $V^{\prime}$ - его объем, и пусть известно, что $12 V^{\prime} \in \mathbb{Q}$ является целым над кольцом, порожденным квадратами длин ребер из $P^{\prime}$. В таком случае любое нормирование на $\mathbb{Q}$, конечное над соответствующим кольцом $R^{\prime}$, конечно и на элементе $12 V^{\prime}$; в частности на $12 V^{\prime}$ конечно и значение рассматриваемого нормирования $\varphi$. Далее, так как объемы $V$ и $V^{\prime}$ связаны соотношением $12 V=12 V^{\prime} \pm 12 V_{0}$, где $V_{0}$ - объем тетраэдра $q p_{i_{0}-1} p_{i_{0}} p_{i_{0}+1}$, на котором значение $\varphi$ тоже конечно, то в силу следствия 3 нормирование $\varphi$ конечно и на $12 \mathrm{~V}$. Следовательно, для проверки целостности $12 \mathrm{~V}$ по критерию с использованием нормирований достаточно проверить целостность объема многогранника $P^{\prime}$ с “хорошей” вершиной $q$ меньшей степени, что и утверждалось. 
Пусть теперь для координат вершин рассматриваемого многогранника $P$ нет выполнения условия их алгебраической независимости. Сначала заметим, что квадраты длин соответствующих ребер различных многогранников с одинаковой комбинаторной структурой являются, по представлению (1), совпадающими элементами поля $\mathbb{Q}\left(x_{1}, \ldots, z_{n}\right)$ независимо от численных значений длин, поэтому многочлен вида (18) для целого элемента $12 V \in \mathbb{Q}$ имеет одинаковую степень $N$ и одинаковые коэффициенты $a_{i}, 1 \leqslant i \leqslant N$, для всех таких многогранников (с алгебраически независимыми координатами вершин) как некоторые многочлены от квадратов длин ребер. Поэтому можно поступать двояко: 1) или, как в доказательстве основной леммы, строим аппроксимации многогранника $P$ многогранниками с тем же комбинаторным строением и с алгебраически независимыми координатами вершин и находим многочлен (6) как предел многочленов с коэффициентами неизменяемого вида от квадратов длин ребер, или же 2) строим отображение $\left(\tilde{x}_{i}, \tilde{y}_{i}, \tilde{z}_{i}\right) \rightarrow\left(x_{i}, y_{i}, z_{i}\right), 1 \leqslant i \leqslant n$, где $\left(\tilde{x}_{i}, \tilde{y}_{i}, \tilde{z}_{i}\right)$ - алгебраически независимые координаты некоторого многогранника $\widetilde{P}$ с тем же комбинаторным строением, что у многогранника $P$, и так как это отображение является гомоморфизмом соответствующих колец, существующее полиномиальное уравнение для целого элемента $12 \operatorname{vol}(\widetilde{P})$ по гомоморфизму перейдет точно в такое же уравнение для $12 \operatorname{vol}(P)$. Лемма доказана.

После установления этой леммы дальнейшее доказательство существования многочлена для объема проводится точно по той же схеме, что и в первом доказательстве.

\section{1. Некоторые выводы}

Как итог, заметим, что каждое из доказательств имеет свой "плюс" и свой "минус". Второе доказательство существенно короче, и именно оно чаще всего используется для публичного изложения доказательства теоремы, но оно дает только сам факт существования многочлена для объема и ничего не говорит о том, как его найти. Первое же доказательство длиннее, но оно конструктивное и вместе с доказательством существования дает и способ построения искомого многочлена для объема.

Более того, многочлен для объема может быть найден без знания какой бы то ни было информации о расположении многогранника в пространстве и даже без знания факта его существования, достаточно знать только его комбинаторное строение и длины его ребер. Это равносильно знанию абстрактно заданной развертки из треугольников, которые должны быть гранями многогранника, т. е. предполагается, что нам задана натуралъная развертка многогранника. Следовательно, если нам задана развертка из треугольников, из которых нужно как-то склеить многогранник с соблюдением заданного комбинаторного его строения, то мы, еще не имея многогранника и даже не зная, можно ли его вообще склеить по данной его натуральной развертке, уже знаем конечный список возможных значений его объема. Из этого наблюдения мы получаем следующее необходимое условие для изометрической реализуемости данной многогранной метрики в виде многогранника в $\mathbb{R}^{3}$. 
ТЕОРема 2. Пусть многогранная метрика задана в виде развертки треугольников, гомеоморфной некоторому ориентируемому многообразию. Тогда для реализуемости этой метрики как метрики многогранника в $\mathbb{R}^{3}$ с гранями из данного набора треугольников, необходимо, чтобы многочлен для квадрата объема, построенный по данной развертке, имел хотя бы один неотрицательный коренъ.

Очевидно, что если мы хотим иметь изометрическое вложсение развертки в $\mathbb{R}^{3}$ с гранями из данной развертки, то необходимо, чтобы многочлен объема имел хотя бы один положительный корень $V^{2}$.

СлЕДСТВИЕ 4. Если многочлен для квадрата оббема многогранника, nостроенный по данной развертке, имеет корни только отрицательные или комплексные, то такую развертку нельзя представить как составленную из граней некоторого многогранника в $\mathbb{R}^{3}$.

ПримеР. Пусть положительные числа $l_{1}, \ldots, l_{6}$ таковы, что правая часть формулы (4) отрицательна. Тогда эти числа не могут быть длинами ребер тетраэдра с указанным на рис. 1 распределением длин ребер, т. е. тогда тетраэдра с данными по модели рис. 1 гранями не существует (конечно, утверждение содержательно только в предположении выполнения соответствующих неравенств треугольника, иначе не будет существования даже и граней).

Отметим, что утверждение следствия 4 никак не пересекается с известной теоремой А. Д. Александрова [37] о реализуемости выпуклой многогранной метрики как метрики некоторого выпуклого многогранника в $\mathbb{R}^{3}$, так как в теореме Александрова априори нет никакой информации или никакого требования о комбинаторном строении искомого многогранника. По той же причине нет пересечения и с теоремой Бураго-Залгаллера [38] о реализуемости в $\mathbb{R}^{3}$ любой многогранной метрики. Вместе с тем следствие 4 дает первый признак нереализуемости многогранной метрики в классе многогранников с фиксированным комбинаторным строением, причем для многогранников любого топологического рода.

\section{2. Канонические многочлены объема}

Как уже упоминалось выше, нахождение многочлена объема по методу самого доказательства теоремы о его существовании приводит к многочленам высокой степени, число корней которых заведомо превосходит число возможных изометрических реализаций данной многогранной метрики в виде многогранников с разными значениями объема. Кроме того, умножение найденного многочлена объема на любой другой приведенный многочлен тоже дает новый многочлен объема бо́льшей степени. Поэтому естественно желание найти многочлен объема как можно меньшей степени, тоже имеющий своими корнями все возможные значения объемов. Напомним (см. конец раздела 5), что многочленом объема для многогранника с данным комбинаторным строением $K$ мы называли любой приведенный многочлен $Q \in \mathbb{Q}[l][V]$ с полиномиально зависящими от переменных $l_{1}^{2}, \ldots, l_{e}^{2}$ коэффициентами, обращающийся в тождественный нуль при подстановке вместо $V$ и $l_{i}^{2}, 1 \leqslant i \leqslant e$ (совокупность квадратов 
длин мы обозначаем здесь и далее через $l$ ) их значений через координаты вершин многогранника ${ }^{13}$. Обозначим через $S$ множество всех таких многочленов, причем даже расширим этот класс, не требуя априори, чтобы их старший коэффициент lс был равен 1. Предположим, что рассматриваются многогранники рода 0 , т.е. гомеоморфные сфере. Оказывается, для таких многогранников среди их многочленов объема можно указать многочлены наименьшей степени, которые мы будем называть каноническими многочленами обгема. Точная формулировка этого утверждения дается следующей теоремой [39].

Теорема 3. Пусть $d$-наименьшая степень ненулевых многочленов из $S$. Тогда среди многочленов из множества $S$ степени $d$ найдется многочлен $Q_{0}$ maкой, что

1) его старший коэффициент $\operatorname{lc}\left(Q_{0}\right)$ равен 1 ;

2) он делит все многочлены из $S$;

3) такой многочлен $Q_{0}$ единственный.

Сначала докажем несколько лемм.

ЛЕмма 4. Если некоторый многочлен $w(l) \in \mathbb{Q}[l]$ не равен тождественно нулю, то при подстановке вместо квадратов длин их значений через координаты вериин по формуле (1) получим многочлен $q(x)=w(l(x)) \not \equiv 0$.

Действительно, гомеоморфный сфере симплициальный многогранник с $n$ вершинами имеет $e=3 n-6$ ребер, а количество координат вершин, однозначно определяющих положение многогранника с точностью до его движения в пространстве, тоже равно $3 n-6$. По теореме Штейница, гомеоморфный сфере многогранник любого комбинаторного строения имеет в пространстве изоморфный ему строго выпуклый многогранник (т. е. с тем же комбинаторным строением). Пусть $x^{0}-$ набор координат вершин некоторого строго выпуклого многогранника с какой-либо закрепленной гранью, а $l^{0}-$ набор квадратов длин ребер этого многогранника. Как показано в [8], отображение $x \rightarrow l$ по формуле (1) в случае строго выпуклых многогранников является диффеоморфизмом. Следовательно, в окрестности точек $x^{0}$ и $l^{0}$ существует обратный диффеоморфизм $l \rightarrow x$, поэтому если $q(x)=w(l(x)) \equiv 0$, то и $w(l)=q(x(l)) \equiv 0$, что противоречит условию $w(l) \not \equiv 0$.

По-другому утверждение доказанной леммы можно сформулировать как алгебраическую независимость многочленов

$$
l_{i j}=\left(x_{i}-x_{j}\right)^{2}+\left(y_{i}-y_{j}\right)^{2}+\left(z_{i}-z_{j}\right)^{2},
$$

заданных через координаты концов ребер гомеоморфного сфере многогранника.

Лемма 5. Пусть $P_{1}, P_{2} \in S$ u $\operatorname{lc}\left(P_{1}\right)=1$. Пусть $R=$ НОД $\left(P_{1}, P_{2}\right) \in \mathbb{Q}(l)[V]$ c $\operatorname{lc}(R)=1$. Тогда

a) $\operatorname{deg} R>0$

b) $R \in \mathbb{Q}[l][V]$.

\footnotetext{
${ }^{13}$ Мы раньше предполагали зависимость многочлена от $V^{2}$, но на самом деле это не нужно, так как такая зависимость многочлена объема от $V$ получается автоматически как следствие его определения.
} 
ДокАзАтЕльство. а) Будем искать наибольший общий делитель $R$ по алгоритму Евклида, т. е. составим последовательность многочленов $T_{n+1}=T_{n-1}-$ $U_{n} T_{n}, n>1$, где $T_{1}=P_{1}, T_{2}=P_{2}$, при этом $\operatorname{deg} T_{n+1}<\operatorname{deg} T_{n}$, а $T_{n}, U_{n} \in$ $\mathbb{Q}(l)[V]$. Параллельно рассмотрим последовательность многочленов $\widetilde{T}_{1}=$ $P_{1}(l(x), V), \widetilde{T}_{2}=P_{2}(l(x), V), \widetilde{T}_{n}(l(x), V) \in \mathbb{Q}(x)[V]$ с соотношениями $\widetilde{T}_{n+1}=$ $\widetilde{T}_{n-1}-\widetilde{U}_{n} \widetilde{T}_{n}$. Так как по лемме 4 ненулевые коэффициенты многочленов $T_{n}(l, V)$ при переходе к координатам $(x)$ превращаются в ненулевые рациональные функции от $(x)$ при тех же степенях $V$, то многочлен $\widetilde{R}(x, V)=R(l(x), V)$ будет НОД для многочленов $\widetilde{P}_{1}(x, V)$ и $\widetilde{P}_{2}(x, V)$. Но для многочленов $\widetilde{P}_{1}(x, V)$ и $\widetilde{P}_{2}(x, V)$ функция $V(x)$ является общим корнем, т. е. оба этих многочлена делятся на $V-V(x)$ в кольце $\mathbb{Q}(x)[V]$, поэтому $\widetilde{R}(x, V)$ тоже делится на $V-V(x)$, значит, $\operatorname{deg} R=\operatorname{deg} \widetilde{R}>0$.

b) По доказанному, $P_{1}$ делится на $R$, поэтому $P_{1}=R W$, где $P_{1} \in \mathbb{Q}[l][V]$, $R, W \in \mathbb{Q}(l)[V]$ и $\operatorname{lc}\left(P_{1}\right), \operatorname{lc}(R), \operatorname{lc}(W)=1$. Покажем, что на самом деле $R \in \mathbb{Q}[l][V]$. Допустим обратное. Тогда в $R(l, V)$ и $W(l, V)$ существуют коэффициенты в виде рациональных дробей с непостоянными знаменателями. Пусть многочлен $f(l)$ - неприводимый делитель в знаменателе какого-нибудь коэффициента в $R$. Рассмотрим коэффициенты $r_{a}$ и $w_{b}$ многочленов $R$ и $W$ соответственно при их $a$-м и $b$-м мономах (считая старший моном нулевым по номеру), в которые $f$ входит в минимальной степени $t_{a}$ и $u_{b}$. По отношению к $R$ это означает, что $a \geqslant 1$ и $t_{a}<0$; а для $u_{b}$ имеем неравенство $u_{b} \leqslant 0$, иначе в $P_{1}=R W$ после умножения $R$ на $W$ получим при $V^{N-a}$ дробный коэффициент. Если таких коэффициентов с минимальными $t_{a}$ и $u_{b}$ несколько, выберем те из них, что при наименьшей степени $V$. Тогда $R$ и $W$ запишутся в виде

$$
\begin{aligned}
R & =V_{M}+f^{t_{1}} A_{1}(l) V^{M-1}+\cdots+f^{t_{a}} A_{a}(l) V^{M-a}+\cdots+f^{t_{M}} A_{M}(l), \\
W & =V^{N-M}+f^{u_{1}} B_{1}(l) V^{N-M-1}+\cdots+f^{u_{b}} B_{b}(l) V^{N-M-b}+\cdots+f^{u_{N-M}} B_{N-M}(l),
\end{aligned}
$$

где $t_{i} \geqslant t_{a}$ при $i<a$ и $t_{i}>t_{a}$ при $i>a$; аналогично, $u_{j} \geqslant u_{b}$ при $j<b$ и $u_{j}>u_{b}$ при $j>b$. Имеем, что в коэффициент многочлена $P_{1}=R W$ при степени $V^{N-a-b}$ множитель $f$ входит в степени $t_{a}+u_{b}<0$, что противоречит предположению $P_{1} \in \mathbb{Q}[l][V]$.

ДоказАТЕЛЬство теоремы 3. 1) Множество $S$ непусто, например, в нем есть многочлен $Q(l, V)$, данный формулой (6) в первом доказательстве основной теоремы, причем его старший коэффициент равен 1. Если этот многочлен единственный в $S$, то он и есть искомый канонический многочлен $Q_{0}$ с утверждаемыми в теореме 3 свойствами. Пусть $S$ содержит еще другие многочлены. Среди них есть ненулевые многочлены (один или больше) наименьшей степени $d$. Выберем из них какой-нибудь многочлен $P$ c $\operatorname{deg} P=d$ и рассмотрим пару многочленов $Q(l, V)$ из (6) и $P$. Применяя к ним лемму 5 , получим, что $R=\operatorname{HOД}(Q, P) \in \mathbb{Q}[l][V]$ и $\operatorname{lc}(R)=1$. А в доказательстве утверждения а) леммы 5 было установлено, что многочлен $R(l(x), V)$ делится на $V-V(x)$ (в кольце $\mathbb{Q}(x)[V])$, значит, $R(l(x), V(x)) \equiv 0$, т. е. $R(l, V) \in S$ и поэтому $\operatorname{deg} R \geqslant d$. Кроме того, $\operatorname{deg} R \leqslant \operatorname{deg} P=d$, значит, $\operatorname{deg} R=d$ и $R$ является искомым многочленом $Q_{0}$. 
2) Возьмем любой многочлен $T \in S$. По лемме 5 и по доказанному в части 1 ) теоремы получаем, что

$$
R_{0}=\text { НОД }\left(Q_{0}, T\right) \in \mathbb{Q}[l][V], \quad \operatorname{lc}\left(R_{0}\right)=1, \quad R_{0} \in S .
$$

Значит, $\operatorname{deg} R_{0} \geqslant d$, с другой стороны, $\operatorname{deg} R_{0} \leqslant \operatorname{deg} Q_{0}=d$, отсюда следует, что $R_{0}=Q_{0}$ и $T$ делится на $Q_{0}=R_{0}$.

3) Пусть в $S$ есть два приведенных многочлена $R_{1}, R_{2}$ с одинаковыми степенями $d$, которые делят все многочлены из $S$. Тогда они делят и друг друга, что возможно только при их равенстве. Теорема доказана.

Тем самым, мы имеем алгоритм нахождения канонического многочлена объема: для этого достаточно взять любой многочлен из множества $S$ (например, многочлен (6), который построен по ходу первого доказательства основной теоремы), разложить его на множители в поле $\mathbb{R}$, и тогда один из множителей со старшим коэффициентом 1 будет искомым каноническим многочленом объема.

Теперь естественно задаться вопросом о существовании и способах нахождения канонического многочлена объема для многогранников произвольного топологического рода $g>0$. K сожалению, в этом случае предыдущие рассуждения не пригодны ввиду несправедливости леммы 4. Действительно, симплициальные многогранники рода $g \geqslant 1$ с $n$ вершинами имеют $e=3 n+6 g-6$ ребер при наличии $3 n-6$ свободных координат вершин, поэтому длины ребер таких многогранников не могут быть выбраны произвольно, а должны подчиняться $6 g \geqslant 6$ условиям. Это значит, что в $e$-мерном пространстве неотрицательных чисел $l_{1}, \ldots, l_{e}$ точки, соответствующие длинам существующих многогранников рода $g$ и данного комбинаторного строения, должны располагаться на некоторой алгебраической поверхности коразмерности $k=6 g$. Иначе говоря, для разрешимости системы, составленной из уравнений (1) для всех ребер многогранника, на правые части должны быть наложены $6 g$ уравнений связи. Если $F_{i}\left(l_{1}, \ldots, l_{e}\right)=0,1 \leqslant i \leqslant k,-$ эти уравнения связи, то при подстановке вместо $l_{j}$ их значений по формуле (1) мы должны получить тождества $F_{i}(l(x)) \equiv 0$. Следовательно, существуют нетривиальные уравнения $w(l)=0$, которым соответствуют тождества $w(l(x)) \equiv 0$, поэтому утверждение леммы 4 оказывается неверным. Таким образом, появляется следующий нерешенный вопрос.

Вопрос 1. Как для многогранников рода $g \geqslant 1$ доказать существование и найти способ построения канонического многочлена объема?

Этот же вопрос приведен в [27; Problem Section].

ЗАмечАниЕ 2. В терминах длин канонический многочлен объема не может быть единственным, так как добавление в любой (нестарший) коэффициент этого гипотетического многочлена $P$ какого-либо нетривиального многочлена от квадратов длин, обращающегося в тождественный нуль при подстановке в него вместо длин формулы (1), оставляет $P$ многочленом объема (по его определению), но изменяет его как многочлен от длин ребер. Может быть также, что существование или способ нахождения канонического многочлена зависят от возможности или невозможности представления многогранника 
как триангулированного топологического многообразия рода $g \leqslant 1$ в так называемой нормальной форме путем его разрезания с использованием разрезов только по ребрам многообразия (другими словами, от того, можно или нельзя собрать $4 g$-угольник из треугольников развертки с попарным отождествлением соответствующих его сторон - это возможно не во всех случаях).

\section{3. Вычисление многочлена объема для некоторых многогранников}

Рассмотрим вопрос о практическом вычислении многочленом объема. В разделе 3 мы нашли такие многочлены для объемов многогранников с $n=4$ и $n=5$ вершинами непосредственно, без применения "большой" теории. Оба полиномиальных уравнения (4) и (5) имеют наименьшую возможную степень: в случае тетраэдра это очевидно, а в случае 5-вершинника существуют многогранники, имеющие две изометрические реализации с двумя разными значениями $V^{2}$, значит, степень уравнения для объема 5-вершинника не может быть меньше 4. Следовательно, (4) и (5) являются каноническими многочленами объема для соответствующих многогранников.

Рассмотрим теперь случай многогранников с $n=6$ вершинами. Пусть сначала мы имеем 5-угольную пирамиду с триангулированным основанием. Она допускает разрезание ее на две части без добавления новых ребер - тетраэдр с объемом $V_{1}$ и 4-угольную пирамиду с объемом $V_{2}$. Общий объем $V$ равен $V_{2}+\varepsilon V_{1}, \varepsilon= \pm 1$. Для квадратов объемов тетраэдра и 4-угольной пирамиды уравнения известны, и легко найти, что возможные значения объема 5-угольной пирамиды будут корнями приведенного многочлена 8-й степени с порядка миллиона мономами, причем эту степень уменьшить нельзя. Аналогичное рассуждение показывает, что телесный многогранник, ограниченный гомеоморфной сфере $(n-1)$-угольной пирамидальной многогранной поверхностью с триангулированным основанием имеет $2^{n-4}$ значений квадрата объема, являющихся корнями многочлена той же степени для $V^{2}$ или степени $2^{n-3}$ для $V$. Точно такая же степень будет и у многочлена объема для так называемых 3-разложимых многогранников, которые получаются из данного тетраэдра последовательным приклеиванием новых тетраэдров к начальному тетраэдру или к получаемым по ходу построения многогранникам (перед приклеиванием предварительно удаляются две конгруэнтные грани и склейка происходит по краям удаленных граней; подробности см. в [32] и [40]). Все эти многочлены являются каноническими многочленами объема.

Для многогранников с $n=6$ вершинами остается нетривиальный случай октаэдра. Если здесь применить, как в [41], конструкцию, предложенную при первом доказательстве основной теоремы, получится многочлен степени $2^{10}=1024$, что заведомо не кажется минимальной степенью. В [42] указан другой способ вычисления объема октаэдра с использованием только его метрики. Опишем кратко идею этого метода.

Пусть на изображенной на рис. 5 комбинаторной схеме октаэдра диагоналям $A_{1} A_{2}, B_{1} B_{2}$ и $C_{1} C_{2}$ соответствуют векторы $\mathbf{X}, \mathbf{Y}$ и $\mathbf{Z}$. Обозначим квадраты длин этих векторов через $x, y$ и $z$, они равны квадратам длин соответствующих диагоналей октаэдра. Введем еще векторы $\mathbf{a}, \mathbf{b}, \mathbf{c}$, идущие соответственно по 


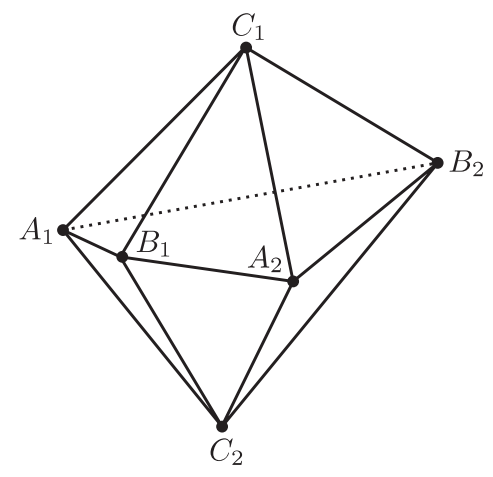

Рис. 5

сторонам $B_{1} C_{1}, C_{1} A_{1}, A_{1} B_{1}$. Вычислим скалярные произведения $(\mathbf{a X}),(\mathbf{a} \mathbf{Y})$, $(\mathbf{a Z}),(\mathbf{b X}),(\mathbf{b} \mathbf{Y}),(\mathbf{b Z})$ и $(\mathbf{X Y}),(\mathbf{X Z}),(\mathbf{Y Z})$. Из рис. 5 имеем представления

$$
\begin{aligned}
& \mathbf{X}=\mathbf{b}+\overrightarrow{C_{1} A_{2}}=\mathbf{a}+\mathbf{c}+\overrightarrow{C_{1} A_{2}}=\mathbf{c}+\overrightarrow{B_{1} A_{2}}, \\
& \mathbf{Y}=\mathbf{a}+\overrightarrow{C_{1} B_{2}}=\mathbf{b}-\mathbf{c}+\overrightarrow{C_{1} B_{2}}=\overrightarrow{B_{1} A_{2}}+\overrightarrow{A_{2} B_{2}}, \\
& \mathbf{Z}=\overrightarrow{B_{1} C_{2}}-\mathbf{a}=\overrightarrow{A_{1} C_{2}}-\mathbf{b}=\overrightarrow{C_{1} A_{2}}+\overrightarrow{A_{2} C_{2}} .
\end{aligned}
$$

Из этих формул получаем, что упомянутые выше скалярные произведения имеют вид

$$
\begin{gathered}
(\mathbf{a X})=(l), \quad(\mathbf{b Y})=(l), \quad(\mathbf{X Y})=(l), \quad(\mathbf{X Z})=(l), \quad(\mathbf{Y Z})=(l), \\
2(\mathbf{b X X})=-x+(l), \quad 2(\mathbf{a Y})=y+(l), \quad 2(\mathbf{a Z})=-z+(l), \quad 2(\mathbf{b Z})=z+(l),
\end{gathered}
$$

где символом $(l)$ обозначены слагаемые, зависящие лишь от квадратов длин ребер октаэдра.

Образуем новые векторы $\mathbf{v}_{a}=-\mathbf{Y}+2 \mathbf{a}+\mathbf{Z}, \mathbf{v}_{b}=-\mathbf{Z}+2 \mathbf{b}+\mathbf{X}$. Для системы из 5 векторов $\left\{\mathbf{v}_{b}, \mathbf{x}, \mathbf{z}, \mathbf{y}, \mathbf{v}_{a}\right\}$ с учетом формул (22) составим матрицу Грама $R$

$$
R=\left(\begin{array}{ccccc}
a_{11}-x-z & a_{12} & a_{13} & a_{14} & a_{15}+z \\
a_{12} & x & a_{23} & a_{24} & a_{25} \\
a_{13} & a_{23} & z & a_{34} & a_{35} \\
a_{14} & a_{24} & a_{34} & y & a_{45} \\
a_{15} & a_{25} & a_{35} & a_{45} & a_{55}-y-z
\end{array}\right),
$$

где $a_{i j}$ - многочлены от квадратов длин ребер октаэдра. Из того, что рассматриваемые векторы лежат в трехмерном пространстве, следует, что все миноры 4-го порядка матрицы $R$ равны нулю. Это нам дает полиномиальные уравнения $R_{11}=\cdots=R_{55}=0$ на переменные $x, y, z$, где $R_{i j}$ обозначают миноры соответствующих элементов.

Пусть $v$ - объем октаэдра. Тогда легко показать, что смешанное произведение $(\mathbf{X}, \mathbf{Y}, \mathbf{Z})$ равно $\pm 6 v$, или

$$
V=V(x, y, z)=(6 v)^{2}=\operatorname{det}\left(\begin{array}{ccc}
x & a_{23} & a_{24} \\
a_{23} & y & a_{34} \\
a_{24} & a_{34} & z
\end{array}\right),
$$


где элементы $a_{i j}$ те же, что и элементы с соответствующими индексами в матрице $R$. Если мы раскроем определитель, то получим

$$
V=x y z+\alpha x+\beta y+\gamma z+\delta,
$$

коэффициенты $\alpha, \beta, \gamma, \delta$ суть многочлены от квадратов длин ребер октаэдра.

Пусть для данного набора длин существует некоторый конкретный октаэдр с диагоналями $\sqrt{x_{0}}, \sqrt{y_{0}}, \sqrt{z_{0}}$. Вычислим значение $V_{0}=V\left(x_{0}, y_{0}, z_{0}\right)$ и, используя многочлены $R_{i j}(x, y, z), V(x, y, z)$, составим 27 многочленов от переменных $(x, y, z)$ :

$$
\begin{aligned}
& V-V_{0}, \quad\left(V-V_{0}\right) x, \quad\left(V-V_{0}\right) y, \quad\left(V-V_{0}\right) z, \quad\left(V-V_{0}\right) x y, \quad\left(V-V_{0}\right) x z, \quad\left(V-V_{0}\right) y z, \\
& \left(V-V_{0}\right) x y z, R_{12}, R_{13}, R_{14}, R_{23}, R_{24}, R_{34}, R_{25}, R_{35}, R_{45}, R_{15}, R_{11} \text {, } \\
& R_{55}, R_{22}, R_{33} R_{44}, R_{15} x, R_{15} y, R_{33} z, R_{15} x y \text {. }
\end{aligned}
$$

Приравняем все эти многочлены к нулю. Полученная система имеет решение $\left(x_{0}, y_{0}, z_{0}\right)$. Смотрим на нее как на однородную линейную систему для 27 переменных

$$
1, x, y, z, x y, x z, y z, x y z, \ldots, x^{2} y^{2} z^{2}
$$

(все эти переменные вида $x^{i} y^{j} z^{k}, 0 \leqslant i, j, k \leqslant 2$, встречаются в первых 8 уравнениях при умножении $V(x, y, z)$ поочередно на $1, x, y, z, x y, x z, y z, x y z)$. Считаем в ней число $V_{0}$ параметром, тогда система может иметь ненулевое решение $\left(1, x_{0}, \ldots, x_{0}^{2} y_{0}^{2} z_{0}^{2}\right)$, только если $Q\left(V_{0}\right)=\operatorname{det} P\left(V_{0}\right)=0$, где $P$ - матрица системы для указанного выше порядка неизвестных. Ее можно представить в виде

$$
P=\left(\begin{array}{ll}
P_{1} & P_{2} \\
P_{3} & P_{4}
\end{array}\right)
$$

где $P_{1}$ - верхнетреугольная матрица размера $8 \times 8$, а матрица $P_{4}$ имеет размер $19 \times 19$. Параметр $V_{0}$ встречается только в матрице $P_{1}$, у которой все диагональные элементы имеют вид $\delta-V_{0}$, поэтому многочлен $\widetilde{Q}\left(V_{0}\right)=\operatorname{det} P\left(V_{0}\right)$ является многочленом 8-й степени от $V_{0}$ со старшим коэффициентом $\operatorname{det} P_{4}$. Первый ключевой момент в дальнейшем доказательстве состоит в следующем: оказывается, $\operatorname{det} P_{4}$, как многочлен от квадратов длин ребер октаэдра, не обращается в тождественный нуль, поэтому в 12 -мерном пространстве $l_{1}, l_{2}, \ldots, l_{12}$ почти для всех точек существует обратная матрица $P_{4}^{-1}$. Тогда мы можем утверждать, что почти для всех значений длин ребер $\operatorname{det} P=\operatorname{det} P_{4} \cdot \operatorname{det}\left(P_{1}-P_{2} P_{4}^{-1} P_{3}\right)$. Действительно, имеем

$$
\left(\begin{array}{ll}
P_{1} & P_{2} \\
P_{3} & P_{4}
\end{array}\right)\left(\begin{array}{cc}
1 & 0 \\
-P_{4}^{-1} P_{3} & 1
\end{array}\right)=\left(\begin{array}{cc}
P_{1}-P_{2} P_{4}^{-1} P_{3} & P_{2} \\
0 & P_{4}
\end{array}\right) .
$$

Второй ключевой момент состоит в том, что элементы матрицы $P_{1}-P_{2} P_{4}^{-1} P_{3}$ оказываются многочленами от квадратов длин ребер октаэдра. Этот приятный факт на самом деле был совсем неожиданным, так как элементы обратной матрицы $P_{4}^{-1}$ являются рациональными функциями (конечно, оба этих ключевых утверждения получаются компьютерными вычислениями).

На основании этих результатов можно утверждать, что равенство $\widetilde{Q}\left(V_{0}\right)=$ $\operatorname{det} P\left(V_{0}\right)=\operatorname{det}\left(P_{1}-P_{2} P_{4}^{-1} P_{3}\right) \operatorname{det} P_{4}=0$ может быть заменено на равенство 
$Q\left(V_{0}\right)=\operatorname{det}\left(P_{1}-P_{2} P_{4}^{-1} P_{3}\right)=0$. В определителе 8-го порядка $\operatorname{det}\left(P_{1}-P_{2} P_{4}^{-1} P_{3}\right)$ ниже главной диагонали переменной $V_{0}$ нет, а элементы $p_{i i}$ на главной диагонали имеют вид $q_{i i}-V_{0}$, где $q_{i i}, 1 \leqslant i \leqslant 8$, не зависят от $V_{0}$. Кроме того, в элементах выше главной диагонали $V_{0}$ встречается только в первой степени. Значит, наивысшая степень $V_{0}$ получается в произведении элементов главной диагонали, т. е. полиномиальное уравнение $Q(V)=0$ имеет вид $V^{8}+a_{1}(l) V^{7}+\cdots=0$, где, напомним, $V=36 v^{2}$, а $v$ есть алгебраический объем октаэдра.

Нетрудно построить пример октаэдра, имеющего 8 изометричных конфигураций с 8 разными значениями $v^{2}$ (для этого достаточно взять так называемый кросс-политоп, являющийся октаэдром, вершины которого расположены симметрично на осях координат, и отразить его 8 разными способами относительно координатных плоскостей - см. рисунки в [11]). Следовательно, найденный многочлен для объема октаэдра является каноническим, так как никакой многочлен меньшей степени не может иметь 8 разных корней $V^{2}$.

Явная запись многочлена для объема октаэдра практически невозможна, так как в общем случае он содержит миллионы, если не миллиарды, мономов. Но для октаэдров Брикара 1-го и 2-го типа, у которых есть попарное равенство длин сторон, вычисления (ввиду возможности приведения большого количества подобных слагаемых) дают многочлены с относительно небольшим количеством слагаемых. Пусть, например, для модели октаэдра на рис. 5 длины сторон даны следующими соотношениями:

$$
\begin{array}{ll}
\left|A_{1} B_{1}\right|^{2}=\left|A_{2} B_{2}\right|^{2}=a, \quad\left|A_{1} B_{2}\right|^{2}=\left|A_{2} B_{1}\right|^{2}=b, \quad\left|B_{1} C_{1}\right|^{2}=\left|B_{2} C_{2}\right|^{2}=c, \\
\left|B_{1} C_{2}\right|^{2}=\left|B_{2} C_{1}\right|^{2}=d, \quad\left|A_{1} C_{1}\right|^{2}=\left|A_{2} C_{2}\right|^{2}=e, \quad\left|A_{1} C_{2}\right|^{2}=\left|A_{2} C_{1}\right|^{2}=f .
\end{array}
$$

Тогда

$$
\begin{aligned}
Q(V)=V^{8}-4[a b(c+d+e+f-a-b)+c d(a+b+e+f-c-d) \\
+e f(a+b+c+d-e-f)-(a c e+a d f+b c f+b d e)] V^{7}=0
\end{aligned}
$$

где $V=36 v^{2}$, а $v$ - объем октаэдра. Корень $V=0$ соответствует объему изгибаемого октаэдра Брикара 1-го типа, а второй ненулевой корень дает формулу объема другой неизгибаемой изометрической реализации октаэдра с теми же длинами сторон, в частности, он дает объем выпуклого октаэдра с указанными длинами сторон. В [39] можно найти явный вид многочлена объема для октаэдра Брикара 2-го типа, который содержит только степени $V^{8}, V^{7}$ и $V^{6}$.

Кроме октаэдров Брикара, существует много других случаев, когда некоторые ребра октаэдров оказываются равными и вместо 12 произвольных длин в вычислении объема участвует меньшее количество свободных длин. Так бывает, например, при наличии в строении октаэдра какой-либо симметрии. В работе [43] введены понятия комбинаторной, метрической и пространственной симметрии многогранников, и после получения полной классификации октаэдров с метрической симметрией - их оказалось 25 основных типов - почти для всех случаев показано, что их многочлены объема имеют сравнительно небольшое количество мономов, и все такие многочлены выписаны в явном виде (исключение составляют лишь два типа с тремя подслучаями, когда число свободных длин ребер равно 7 или 8). Общим для всех явно найденных 
многочленов оказалось то, что все они имеют вид

$$
V^{8}+a V^{7}+b V^{6}=0
$$

так что возможные значения объемов можно вычислить в явном виде (есть даже случаи, когда $b=0)$. Следует заметить, что среди корней этих многочленов, согласно теореме 1, находятся все возможные значения октаэдров с данной метрической симметрией, в том числе и тех, конфигурация которых не обладает пространственной симметрией.

И в общем случае симметрия или малое количество различных длин ребер многогранника должно существенно облегчить вычисление многочлена для его объема. В частности, было бы интересно найти канонический многочлен объема для многогранника Штеффена - простейшего изгибаемого многогранника без самопересечений. Он имеет 9 вершин, 21 ребро и 5 различных значений длин ребер. Его описание можно найти во многих работах (см., например, [11]).

Умение вычислять объемы многогранников без предварительного знания их пространственной формы, а зная только их комбинаторику и длины ребер, может оказаться полезным в некоторых вопросах не только математики, но и естествознания и техники. Например, в теории растворов для исследования их термодинамических свойств важно знать суммарный объем молекул растворенной соли, образованных из нескольких стандартных комбинаций атомов, имеющих, как следствие постоянства некоторых физико-химических связей между ними, постоянство расстояний. В случае, рассмотренном в работе [44], молекулы имеют комбинаторное строение октаэдра с некоторой метрической симметрией и с двумя или тремя разными длинами ребер, что дало возможность вычислить объемы молекул в разных вариантах расположения составляющих их атомов.

ЗАмЕчАНИЕ 3. В связи с поиском возможных значений объемов многогранника с известной метрикой полезно заметить следующее: существуют изометричные многогранники с одинаковым комбинаторным строением, один выпуклый, другой невыпуклый, причем невыпуклый имеет бо́льший объем. Этот феномен был показан в работе [38] путем довольно сложных построений с привлечением многогранников с очень большим количеством вершин, но оказалось, что такое возможно даже для многогранников с комбинаторным строением октаэдров. Построение этого примера дано в работе [45], а соответствующие октаэдры с точными координатами вершин можно увидеть в Интернете на сайте http://www. etudes.ru/ru/mov/mov002/index.php.

\section{4. Вычисление длин диагоналей многогранников}

Возможность вычисления объема многогранника лишь на основе знания его комбинаторного строения и метрики позволяет найти много других его геометрических параметров. В этом разделе мы, используя результаты работы [46], покажем, как найти диагонали многогранника.

Рассмотрим произвольные две грани многогранника с общим ребром. Если две вершины этих граней, не инцидентные их общему ребру, не соединены ребром, то диагональ многогранника, которую можно провести между ними, 
назовем малой диагональю. Ради краткости речи, мы часто будем называть малой диагональю и длину отрезка (или расстояние), мысленно соединяющего эти вершины. Справедлива следующая теорема.

ТЕоремА 4. Для каждой малой диагонали есть полиномиальное уравнение, коэфбициенты которого зависят только от выбора этой малой диагонали, метрики и комбинаторного строения многогранника, и для многогранников в общем положении они не все равны нулю.

Мы не будем приводить здесь полное доказательство теоремы (так как она доказана подробно в [46]), а докажем сначала лишь ключевую для нее лемму и затем изложим только схему доказательства.

ЛЕмма 6. Пусть $l_{k}$ - длина произвольным образом выбранного ребра в многогранниках данного комбинаторного строения $K$. Тогда не может бытъ, чтобъ между длинами ребер этих многогранников существовала зависимость вида ${ }^{14}$

$$
L\left(\hat{l}_{k}, l\right)=A_{0}\left(\hat{l}_{k}\right) l_{k}^{S}+\cdots+A_{S-1}\left(\hat{l}_{k}\right) l_{k}+A_{S}\left(\hat{l}_{k}\right)=0
$$

такая, что $A_{0}^{2}\left(\hat{l}_{k}\right)+\cdots+A_{S-1}^{2}\left(\hat{l}_{k}\right) \neq 0$ nри $l_{1}^{2}+\cdots+l_{k-1}^{2}+l_{k+1}^{2}+\cdots+l_{e}^{2} \neq 0$, где е - число ребер, а запись $\hat{l}_{k}$ означает, что в аргументе участвуют все длины $l_{1}, \ldots, l_{e}$, кроме $l_{k}$.

ДокАЗАТЕЛЬСтво ЛЕммы 6. Действительно, пусть в $K$ концевые вершины $k$-го ребра имеют номера $i$ и $j$, а прилегающие к этому ребру грани $F$ и $G$ имеют вершины $i, j, p$ и $i, j, q$. Построим в $\mathbb{R}^{3}$ многогранник $P(K)$ по следующему отображению $P: K \rightarrow \mathbb{R}^{3}$ : вершинам $i, j, p$ и $q$ сопоставим соответственно точки $M_{i}\left(0, y_{i}, z_{i}\right), M_{j}\left(0, y_{j}, z_{j}\right), M_{p}(a, 0,0)$ и $M_{q}(-a, 0,0), a \neq 0$, а все остальные вершины отобразим в некоторые точки на оси $O x$. Теперь будем вращать точки $M_{i}$ и $M_{j}$ вокруг оси $O x$, оставляя все остальные вершины на месте. В процессе такой деформации будем получать новые многогранники $P_{t}$ с тем же комбинаторным строением $K$. При этом длины всех ребер, кроме $k$-го, не изменяются, и поэтому наличие уравнения (27) с указанным в условиях леммы свойством означало бы, что при данных значениях длин $l_{1}, \ldots, l_{k-1}, l_{k+1}, \ldots, l_{e}$, не все из которых равны нулю, длина $l_{k}$ могла бы иметь только некоторое конечное число значений, что невозможно ввиду непрерывного изменения $l_{k}$ в процессе вращения многогранника. Лемма доказана.

Изложим теперь идею доказательства теоремы 4. Пусть $\langle C D\rangle$ - малая диагональ в многограннике $P$ для двугранного угла между гранями $\langle A B C\rangle$ и $\langle A B D\rangle$ с общим ребром $\langle A B\rangle$. Выбросим эти две грани и заклеим образовавшуюся дыру двумя новыми гранями $\langle A D C\rangle$ и $\langle B D C\rangle$; получим новый многогранник $P_{1}$, для которого малая диагональ $\langle C D\rangle$ многогранника $P$ является одним из ребер (рис. 6). Так же как объем $V=\operatorname{vol}(P)$ является корнем многочлена (6), объем

${ }^{14}$ Тот факт, что уравнение (27) выполняется для длин ребер именно многогранников, а не для длин одномерных симплексов комплекса $K$, означает, что подстановка в (4) значений $l=l(x)$ из (1) обращает это уравнение в тождество $L\left(\hat{l}_{k}(x), l(x)\right) \equiv 0$. 


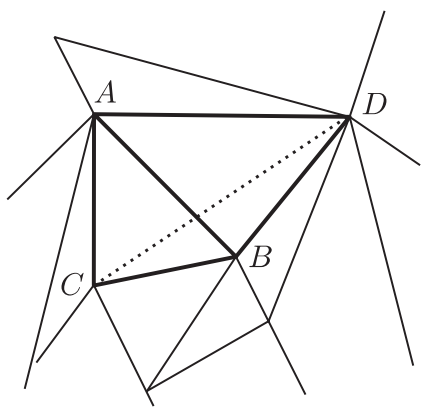

многогранник $P$

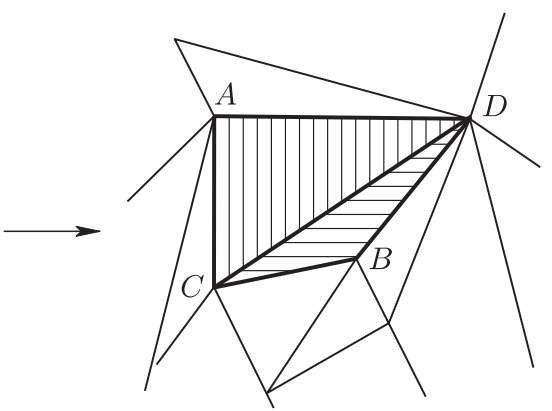

многогранник $P_{1}$

Рис. 6

$V_{1}=\operatorname{vol}\left(P_{1}\right)$ является корнем некоторого уравнения вида ${ }^{15}$ :

$$
Q_{1}=V_{1}^{2 N}+a_{1}(\hat{l}, d) V_{1}^{2 N-2}+\cdots+a_{N}(\hat{l}, d)=0
$$

в котором коэффициенты $a_{i}, 1 \leqslant i \leqslant N$, зависят от длин ребер многогранника $P$ (но не от длины ребра $\langle A B\rangle$, что отражено в обозначении $\hat{l}$ ) и длины $d$ диагонали $\langle C D\rangle$. Объемы $V=\operatorname{vol}(P)$ и $V_{1}=\operatorname{vol}\left(P_{1}\right)$ отличаются на геометрический объем $V_{T} \geqslant 0$ тетраэдра $\langle A B C D\rangle$ :

$$
V_{1}=V+\varepsilon V_{T}, \quad \varepsilon= \pm 1
$$

Так как в общем случае неизвестно, надо ли объем тетраэдра прибавить или отнять, то необходимо найти соотношение между $V$ и $V_{1}$, справедливое при любой ориентации и при любом взаимном расположении рассматриваемых многогранников. Для этого исключаем $\varepsilon$ и получаем уравнение:

$$
V_{1}^{4}-2\left(V^{2}+V_{T}^{2}\right) V_{1}^{2}+\left(V^{2}-V_{T}^{2}\right)^{2}=0
$$

Рассматривая (28) и (29) как уравнения относительно общей переменной $v=V_{1}^{2}$, мы должны иметь равенство нулю их результанта $R$ :

$$
R=\left|\begin{array}{ccccccc}
1 & a_{1} & a_{2} & a_{3} & \ldots & a_{N} & 0 \\
0 & 1 & a_{1} & a_{2} & \ldots & a_{N-1} & a_{N} \\
1 & -2\left(V^{2}+V_{T}^{2}\right) & \left(V^{2}-V_{T}^{2}\right)^{2} & 0 & \ldots & 0 & 0 \\
\vdots & \vdots & \vdots & \ddots & \vdots & \vdots & \vdots \\
\vdots & \vdots & \vdots & \vdots & \ddots & \vdots & \vdots \\
0 & 0 & 0 & \cdots & \cdots & \left(V^{2}-V_{T}^{2}\right)^{2} & 0 \\
0 & 0 & 0 & \cdots & \cdots & -2\left(V^{2}+V_{T}^{2}\right) & \left(V^{2}-V_{T}^{2}\right)^{2}
\end{array}\right|=0
$$

\footnotetext{
${ }^{15}$ Степень многочлена $Q_{1}$ в общем случае, конечно, другая чем в (6), скажем, $2 N_{1}$, но чтобы избежать обилия индексов, мы оставили то же обозначение $2 N$.
} 
Объем тетраэдра (см. рис. 7) вычисляется по уже известной нам формуле ${ }^{16}$

$$
\begin{aligned}
144 V_{T}^{2}=L^{2} & d^{2}\left(l_{1}^{2}+l_{3}^{2}+l_{2}^{2}+l_{4}^{2}-L^{2}-d^{2}\right) \\
& +l_{1}^{2} l_{4}^{2}\left(L^{2}+d^{2}+l_{2}^{2}+l_{3}^{2}-l_{1}^{2}-l_{4}^{2}\right)+l_{3}^{2} l_{2}^{2}\left(L^{2}+d^{2}+l_{1}^{2}+l_{4}^{2}-l_{3}^{2}-l_{2}^{2}\right) \\
& -L^{2}\left(l_{1}^{2} l_{3}^{2}+l_{2}^{2} l_{4}^{2}\right)-d^{2}\left(l_{1}^{2} l_{2}^{2}+l_{3}^{2} l_{4}^{2}\right)
\end{aligned}
$$

(обозначения длин понятны из рисунка). Если мы раскроем определитель в (30) и соберем слагаемые при степенях $d$, учитывая формулу (31), то получим относительно $d$ некоторый многочлен вида

$$
A_{0}(\hat{l}, L, V) d^{2 K}+A_{1}(\hat{l}, L, V) d^{2 K-2}+\cdots+A_{K}(\hat{l}, L, V)=0 .
$$

Дальнейшие рассмотрения направлены на то, чтобы показать, что в уравнении (32) при ненулевых степенях $d$ найдется хотя бы один коэффициент, который при подстановке в него значений $l$ и $V$ как функций координат вершин многогранника не обращается в тождественный нуль. Для этого используется,

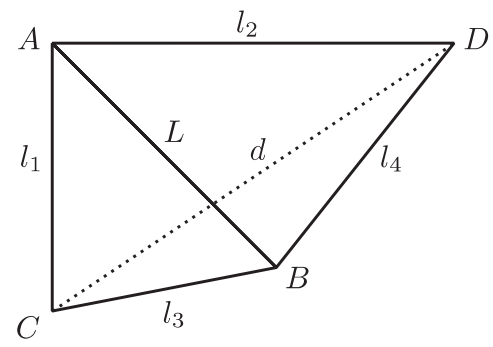

Рис. 7

во-первых, явная зависимость коэффициентов уравнения (32) от $L$ с учетом формул (30) и (31), и, во-вторых, знание структуры общего члена результанта. Напомним, что по теореме о весе общий $j$-й член (моном) результанта (30) имеет вид

$$
c_{j} a_{0}^{\mu_{0}^{(j)}} a_{1}^{\mu_{1}^{(j)}} \cdots a_{N}^{\mu_{N}^{(j)}} b_{0}^{\nu_{0}^{(j)}}(-2)^{\nu_{1}^{(j)}}\left(V^{2}+V_{T}^{2}\right)^{\nu_{1}^{(j)}}\left(V^{2}-V_{T}^{2}\right)^{2 \nu_{2}^{(j)}} \quad\left(a_{0}=b_{0}=1\right)
$$

где $c_{j}$ - некоторое отличное от нуля целое число, а неотрицательные целые числа $\mu_{0}^{(j)}, \mu_{1}^{(j)}, \ldots, \nu_{2}^{(j)}$ удовлетворяют соотношениям

$$
\begin{gathered}
\mu_{0}^{(j)}+\mu_{1}^{(j)}+\cdots+\mu_{N}^{(j)}=2, \quad \nu_{0}^{(j)}+\nu_{1}^{(j)}+\nu_{2}^{(j)}=N \\
\mu_{1}^{(j)}+2 \mu_{2}^{(j)}+\cdots+N \mu_{N}^{(j)}+\nu_{1}^{(j)}+2 \nu_{2}^{(j)}=2 N
\end{gathered}
$$

В итоге после довольно кропотливых рассуждений (см. [46]) находим, что если бы все коэффициенты при степенях $d$ в уравнении (32) были тождественно

${ }^{16} \mathrm{~B}$ формуле (8) работы [46] допущена досадная ошибка: в двух последних слагаемых коэффициенты при $L^{2}$ и $d^{2}$ должны быть переставлены; также в формуле (11) вместо показателя $\nu_{0}^{(j)}$ должно быть $\nu_{1}^{(j)}$, а в последнем уравнении формулы $(12)$ вместо $\mu_{2}^{(j)}$ должно быть $\nu_{2}^{(j)}$. 
равны нулю, то оказалось бы, что $L$ - длина ребра $A B$ - должна обращать в тождество уравнение вида

$$
c_{0} L^{16 N^{2}}+c_{1}(\hat{l}) L^{16 N^{2}-2}+\cdots+c_{8 N^{2}}(\hat{l})=0, \quad c_{0} \neq 0,
$$

т. е. в многогранниках такого комбинаторного строения $K$, как у $P$, одно ребро определяется значениями других ребер, что по лемме 6 невозможно. Тем самым не может быть, чтобы многочлен (32) относительно диагонали $d$ был нулевым. Этим в [46] завершается доказательство теоремы 4.

ЗАмЕчАниЕ 4. При вычислении малых диагоналей можно и не обращаться к значениям объема многогранника. Достаточно вместо этого исключить переменную $V$ из уравнений (6) и (32) и получить полиномиальное уравнение степени $2 K N$ относительно $d^{2}$ с коэффициентами, зависящими только от длин ребер. Но при этом возрастет число ненужных вычислений, так как при использовании объемов мы учитываем толъко неотрицательные значения корней $V^{2}$ уравнения (6) и поэтому число значений $d^{2}$ получается, вообще говоря, меньше чем $2 K N$.

Мы хотим подчеркнуть, что, в отличие от теоремы 1 , в существующем по теореме 4 многочлене (32) для малой диагонали $d$ старший коэффициент не является постоянным числом, а, как и все коэффициенты, является некоторым многочленом от квадратов длин ребер многогранника, и бывают случаи, когда для некоторых конкретных значений длин и объемов все коэффициенты этого многочлена равны нулю. Так должно быть, например, если многогранник изгибается с изменением выбранной малой диагонали.

Из теоремы 4 и приведенного комментария к ней немедленно получаем такие следствия.

СлЕДСТВИЕ 5. Для многогранников в общем положении с данным комбинаторным строением и с данными значениями длин ребер малье диагонали могут принимать только конечное число значений, определяемых как корни уравнений вида (32), в каждое из которых вместо $V^{2}$ подставлены поочередно возможные значения квадрата обгема многогранника, получаемые как неотрицательные корни уравнения (6) относительно $V^{2}$.

СлеДСТВИЕ 6. Для того чтобы многогранник был изгибаемым, необходимо, чтобы хотя бы для одной малой диагонали в соответствующем уравнении (32) все коэфбициенты $A_{i}(l, V), 0 \leqslant i \leqslant K$, при значениях длин ребер и обгема этого многогранника были равны нулю.

СлеДСТВИЕ 7. Почти все многогранники в $\mathbb{R}^{3}$ являются неизгибаемыми.

Утверждение следствия 7 в случае многогранников рода $g=0$ доказано в [8], для рода $g=1$ и выше оно известно только в препринтных публикациях: в [47] и [48] для случая $g=1$, а для общего случая - в [49] с очень сложным доказательством (но в любой размерности).

Вопрос 2. Было бы интересно доказать утверждение следствия 7 тем же способом, каким оно получено в работе [8] - с использованием жесткости 1-го порядка почти всех многогранников рода 0 (по причине жесткости всех строго 
выпуклых многогранников), а у нас для этого надо будет доказать жесткость 1-го порядка почти всех многогранников произвольного рода. Для получения того же результата также было бы достаточно доказать жесткость 2-го порядка почти всех многогранников произвольного рода. Быть может, этот подход даст доказательство неизгибаемости почти всех многогранников и в многомерном случае.

Теперь мы опишем процедуру нахождения длин других диагоналей многогранника в случае, когда мы уже знаем малые диагонали. Основой для этого является формула для расстояния между двумя точками с известными расстояниями до трех базовых точек, не лежащих на одной прямой [50]. Длину ребра $\left\langle p_{i} p_{j}\right\rangle$ обозначим через $l_{i j}$ (см. рис. 8). Далее, пусть $S_{123}$ - площадь тре-

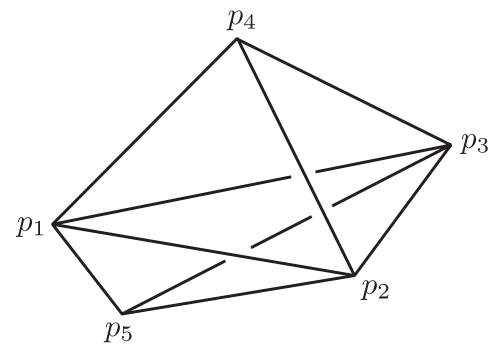

Рис. 8

угольника $\left\langle p_{1} p_{2} p_{3}\right\rangle, V_{1234}$ и $V_{1235}$ - объемы соответствующих тетраэдров. Тогда расстояние $d_{45}$ между вершинами $p_{4}$ и $p_{5}$ вычисляется по формуле

$$
d_{45}^{2}=l_{34}^{2}+l_{35}^{2}+\frac{D+36 \varepsilon V_{1234} V_{1235}}{S_{123}^{2}},
$$

где

$$
D=\frac{1}{8}\left|\begin{array}{ccc}
2 l_{23}^{2} & l_{12}^{2}-l_{23}^{2}-l_{13}^{2} & l_{25}^{2}-l_{35}^{2}-l_{23}^{2} \\
l_{12}^{2}-l_{23}^{2}-l_{13}^{2} & 2 l_{13}^{2} & l_{35}^{2}+l_{13}^{2}-l_{15}^{2} \\
l_{24}^{2}-l_{23}^{2}-l_{34}^{2} & l_{13}^{2}-l_{34}^{2}-l_{14}^{2} & 0
\end{array}\right|,
$$

a $\varepsilon= \pm 1$ в зависимости от того, лежат вершины $p_{4}$ и $p_{5}$ по разные или по одну сторону от плоскости треугольника $\left\langle p_{1} p_{2} p_{3}\right\rangle$ (если одна из вершин $p_{4}$ и $p_{5}$ или обе они лежат на плоскости основания, то соответствующие объемы равны нулю и выбор величины $\varepsilon$ значения не имеет).

Пусть требуется найти длину диагонали между двумя вершинами, принадлежащими краю одной звезды (рис. 9), скажем, расстояние между вершинами $M_{1}$ и $M_{5}$. Расстояния $M_{1} M_{3}, M_{2} M_{4}$ и $M_{3} M_{5}$ известны как длины малых диагоналей. Определяем теперь расстояние между $M_{1}$ и $M_{4}$ по формуле (35) как расстояние между точками с известными расстояниями до вершин базового треугольника $\left\langle O M_{2} M_{3}\right\rangle$, после этого по той же формуле находим расстояние между $M_{1}$ и $M_{5}$ как между точками с известными расстояниями до базовых точек $O, M_{3}$ и $M_{4}$. Очевидно, что таким образом мы можем последовательно определить длины всех диагоналей в одной звезде и заодно отбросить неподходящие выборы длин малых диагоналей, так как каждую "не малую" диагональ 
можно вычислить двумя способами, идя к рассматриваемой вершине по двум разным направлениям и требуя совпадения результатов вычислений.

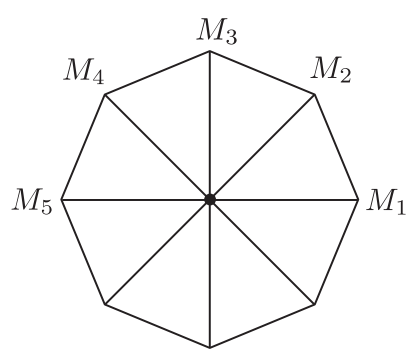

Рис. 9

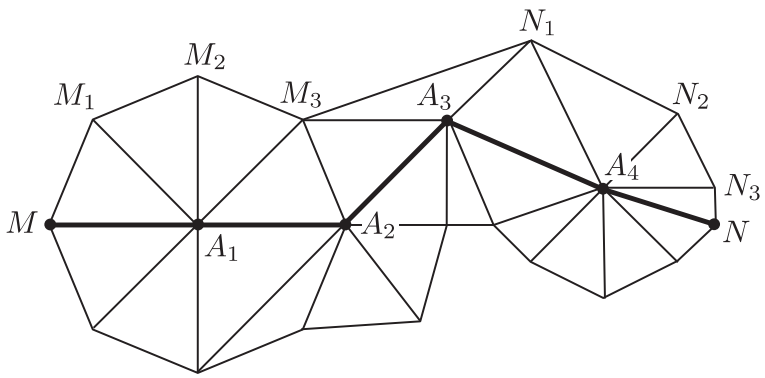

Рис. 10

Пусть теперь диагональ соединяет вершины из разных звезд. Опишем алгоритм вычисления ее длины на примере из рис. 10 для диагонали $M N$. Найдем кратчайший (по числу ребер) путь между точками $M$ и $N$ (на рисунке это ломаная $\left.M A_{1} A_{2} A_{3} N\right)$. Расстояния между вершинами внутри одной звезды мы умеем вычислять. Покажем, как найти расстояния от $M$ до вершин звезды $A_{2}$. Звезды вершин $A_{1}$ и $A_{2}$ имеют общее ребро $\left\langle A_{1} A_{2}\right\rangle$ и общую грань $\left\langle A_{1} A_{2} M_{3}\right\rangle$, которая является базовым треугольником для определения расстояния между $M$ и $A_{3}$ по формуле (35), так как все расстояния от них до вершин грани $\left\langle A_{1} A_{2} M_{3}\right\rangle$ известны. Нетрудно проверить, что грань $\left\langle A_{2} A_{3} M_{3}\right\rangle$ - базовая для определения расстояния между $M$ и $N_{1}$; после определения этого расстояния грань $M_{3} A_{3} N_{1}$ становится базовой для определения расстояния $M A_{4}$. Наконец, известные расстояния от $M$ и $N$ до вершин грани $\left\langle A_{3} A_{4} N_{1}\right\rangle$ позволяют определить расстояние между $M$ и $N$. Конечно, не надо забывать, что на каждом шаге мы находим два возможных значения вычисляемых расстояний, поэтому в итоге получаем не точные значения расстояний, а некоторый конечный набор их возможных значений, что и достаточно для конечности алгоритма. Как видим, алгоритм работает в предположении, что в многограннике нет трех вершин, лежащих на одной прямой, иначе какой-нибудь базовый треугольник окажется вырожденным и не удастся применить формулу (35).

Описанная процедура нахождения диагоналей многогранника приводит к следующей проблеме. Мы знаем, что существуют пары изометричных многогранников, один из которых изгибается, а другой - нет (достаточно рассмотреть изгибаемый октаэдр Брикара $P$ 1-го или 2-типа с плоским экватором и изометричный ему вложенный октаэдр, полученный зеркальным отражением одной половинки $P$ относительно плоскости экватора). Попытка применения указанного выше метода диагоналей даст положительный результат в случае неизгибаемости многогранника, а тот же метод в применении к изгибаемому многограннику "не сработает", так как один из многочленов для малой или "не малой" диагонали окажется состоящим из нулевых коэффициентов. Тогда естественно поставить вопрос: сколько и какие диагонали многогранника считать известными (точнее, зафиксированными), чтобы в этом предположении можно было локально однозначно найти все его остальные диагонали и, тем 
самым, в некоторой достаточно малой окрестности данного многогранника зафиксировать его пространственное положение (другими словами, установить его локальную однозначную определенность или неизгибаемость при фиксации длин указанных диагоналей)? Если достаточное для этого минимальное число фиксируемых (в смысле значения длины) диагоналей равно $p$, то мы называем такой многогранник комбинаторно р-параметрическим. Исследованию этого понятия и его модификаций посвящены работы [51]-[53] и другие указанные в этих работах публикации. Одной из основных нерешенных задач в этом направлении исследований является нахождение алгоритма для вычисления длин диагоналей неизгибаемых многогранников - поскольку многогранник неизгибаемый, значит, локально он определен однозначно своей комбинаторной структурой и метрикой, поэтому определены однозначно и длины его диагоналей - а как их найти? Например, поскольку по теореме Коши выпуклый многогранник однозначно определен своим комбинаторным строением и метрикой, то в классе выпуклых многогранников он является 0-параметрическим, значит, длины его диагоналей должны быть определены однозначно - но каков алгоритм их вычисления?

\section{5. Обобщение уравнений для объема и диагоналей}

После того как установлено, что мы можем вычислять длины всех диагоналей как корни некоторых полиномиальных уравнений, зависящих от комбинаторного строения многогранника и от квадратов длин ребер, следующая теорема из [54] представляется естественным обобщением этих уравнений.

Теорема 5. Пусть $f\left(x_{1}, \ldots, z_{n}\right)$ - многочлен от координат $n$ вершин гомеоморфного сфере симплициального многогранника, инвариантный относительно движений пространства. Тогда существует многочлен $q \in \mathbb{Q}[l, t]$ такой, что $f$ является корнем этого многочлена.

Например, алгебраический объем $V$ многогранника, как мы его определили в разделе 2, является однородным многочленом степени 3 от координат $x_{1}, \ldots, z_{n}$. Он инвариантен относительно движений 1-го рода, а его квадрат инвариантен относительно всех движений и поэтому теорема 5 подтверждает уже известный нам факт существования полинома

$$
c_{0}(l) t^{N}+c_{1}(l) t^{N-1}+\cdots+c_{N}(l)
$$

для которого $V^{2}$ является корнем. То же самое верно и для квадрата длины любой диагонали многогранника. Но теорема 5 ничего не утверждает о виде старшего коэффициента, и доказательство равенства его единице для многочлена в случае $f=V^{2}$ представляло основную трудность.

ДокАЗАТЕЛЬСТво тЕОРЕмы 5 получается как следствие уже использованной в разделе 12 алгебраической независимости квадратов длин ребер гомеоморфного сфере многогранника в кольце многочленов от координат вершин, инвариантных относительно движений. Используя инвариантность многочлена $f$ относительно движений многогранника, расположим многогранник так, 
чтобы вершины одной его грани, скажем, с номерами $1,2,3$, имели координаты $x_{1}=y_{1}=z_{1}=y_{2}=z_{2}=z_{3}=0$. Тогда многочлен $f$ превратится в многочлен $F$, зависящий от $n-6$ свободных координат $x_{2}, x_{3}, y_{3}, x_{4}, \ldots, z_{n}$. Полиномы (1) алгебраически независимы и их число тоже равно $3 n-6$, поэтому они составляют базис трансцендентности в алгебре многочленов от указанных выше переменных [55]. Следовательно, многочлен $F$ является алгебраически зависимым от полиномов (1), что и утверждалось.

Более интересным и более трудным является результат о степени полинома $q$ для данного многочлена $f$. В той же работе [54] доказывается, что среди существующих для $f$ полиномиальных уравнений

$$
q=c_{0}(l) f^{N}+\cdots+c_{N-1}(l) f+c_{N}(l)=0,
$$

вне зависимости от вида многочлена $f$, можно найти полином степени $N \leqslant 2^{e}$, где $е$ равно числу ребер многогранника.

\section{6. Многогранники с известными диагоналями}

Если у ориентируемого симплициального многогранника известны длины не только ребер, но и диагоналей, то можно предложить явную формулу для вычисления его объема. Действительно, расположим начало координат в одной из вершин многогранника и обозначим ее номером 0. Имеем $x_{0}=y_{0}=z_{0}=0$. Пусть $\left(x_{i}, y_{i}, z_{i}\right), 1 \leqslant i \leqslant n,-$ координаты остальных $n$ вершин многогранника. Пусть на многограннике выбрана некоторая ее ориентация. Построим тетраэдры с вершиной в начале координат и с основаниями на гранях многогранника. Тогда обобщенный объем $V$ многогранника выражается формулой

$$
6 V=\sum_{F} \operatorname{det}\left(\begin{array}{lll}
x_{i} & y_{i} & z_{i} \\
x_{j} & y_{j} & z_{j} \\
x_{k} & y_{k} & z_{k}
\end{array}\right),
$$

где суммирование идет по всем граням, а вершины грани в каждом слагаемом расположены в порядке, определяемом ориентацией грани. Возведем равенство (36) в квадрат, причем определители в правой части возьмем один раз в исходном виде, а один раз - в виде транспонированных определителей. Тогда получим

$$
36 V^{2}=\sum_{F_{i j k}} \sum_{F_{p q s}}\left|\begin{array}{ccc}
\mathbf{r}_{i} \mathbf{r}_{p} & \mathbf{r}_{i} \mathbf{r}_{q} & \mathbf{r}_{i} \mathbf{r}_{s} \\
\mathbf{r}_{j} \mathbf{r}_{p} & \mathbf{r}_{j} \mathbf{r}_{q} & \mathbf{r}_{j} \mathbf{r}_{s} \\
\mathbf{r}_{k} \mathbf{r}_{p} & \mathbf{r}_{k} \mathbf{r}_{q} & \mathbf{r}_{k} \mathbf{r}_{s}
\end{array}\right|
$$

(правило суммирования очевидно). Каждое скалярное произведение в элементах матриц в правой части выражается через квадраты длин ребер или диагоналей. Например,

$$
\mathbf{r}_{i} \mathbf{r}_{p}=\frac{\mathbf{r}_{i}^{2}+\mathbf{r}_{p}^{2}-d_{i p}^{2}}{2}
$$

где $\mathbf{r}_{i}^{2}, \mathbf{r}_{p}^{2}$ - квадраты длин диагоналей, соединяющих вершины с номерами $i$ и $p$ с вершиной с номером 0, a $d_{i p}^{2}$ - квадрат длины ребра или диагонали, в соответствии с тем, являются $i$ и $p$ концевыми точками ребра или нет. 
Формула (36) дает точное значение объема как функции длин ребер, если в многограннике нет диагоналей. Простейший пример - формула (4) для объема тетраэдра. Другой пример - тор с наименьшим количеством вершин. Такой тор имеет 7 вершин, соединенные друг с другом ребрами. Формула для объема $V$ такого многогранника была найдена (конечно, с использованием компьютера) студентом МГУ О. Давыдовым в его дипломной работе (1999 г.) и занимает три с половиной страницы формата А4 - но это точная формула вида $V^{2}=\cdots$, не подлежащая упрощению! ${ }^{17}$ (Отметим следующий важный факт - имея каркас, т. е. 1-скелет из 21 ребра, мы можем "обшить" этот 1-скелет треугольными гранями 120 способами и получить 120 торов-многогранников с разным комбинаторным строением и, соответственно, получить 120 (!) формул для их объемов, см., например, [56].) В общем случае можно легко получить верхнюю оценку количества мономов в правой части формулы (37): оно не превышает $243(2 n-2+4 g-k)^{2}$, где $g$ - род многогранника, $k$ - степень вершины, выбранной в качестве начала координат, т. е. существенно меньше, чем количество мономов в многочлене объема без участия диагоналей. Формула вида (37) была известна еще Штаудту [57], и она прокомментирована ${ }^{18}$ и обобщена в [58] Сильвестером.

\section{7. Проблема изометрического погружения многогранной метрики}

Одной из основных проблем метрической теории многогранников является проблема изометрической реализации данной многогранной метрики. Эта задача имеет много постановок. Если искать погружение метрики в виде некоторой многогранной поверхности без какого-либо априорного требования на ее комбинаторное или внешнее строение, то решение такой задачи существует и оно дано в работе [38]. Если заранее предполагать, что метрика задана на топологической сфере и является выпуклой, то она реализуется выпуклым многогранником, причем комбинаторное строение многогранника может не иметь ничего общего с комбинаторным строением развертки (это - классический результат А. Д. Александрова [37]; современные алгоритмы построения, выделяющие в итоге ребра и грани, предложены в работах [59] и [60]). Теорема 4 и ее следствие 5 позволяют предложить следующий алгоритм построения многогранников по их данной натуральной развертке: находятся все неотрицательные корни $V^{2}$ уравнения (6), затем они по очереди подставляются в каждое из уравнений (32) (в общем случае их число равно $e$ - числу ребер многогранника), находятся неотрицательные корни $d^{2}$ всех этих уравнений и, тем самым, составляется список всех возможных значений длин всех малых диагоналей, затем по этому списку составляется новый список, представляющий собой все варианты длин совокупности малых диагоналей для каждого искомого многогранника (т. е. для каждой малой диагонали берется одно

\footnotetext{
${ }^{17}$ Объем ее записи можно немножко уменьшить, если длину ребра обозначать не символом вида $d_{i j}$, а одной буквой, а таких букв потребуется 21.

${ }^{18}$ Штаудт доказал, что произведения площадей двух многоугольников или объемов двух многогранников являются целыми алгебраическими функциями, т. е. многочленами от квадратов всевозможных расстояний между их вершинами, и эти утверждения Сильвестер назвал прекрасными и важными геометрическими теоремами.
} 
из возможных значений ее длины) ${ }^{19}$, и, наконец, простым перебором из этого списка выделяются согласованные длины диагоналей или доказывается, что такого согласованного выбора диагоналей нет, т. е. данная развертка не допускает изометрической реализации в $\mathbb{R}^{3}$. В [46] приведен численный пример реализации этой схемы, выполненный С. Н. Михалёвым. Этот пример относится к реализации развертки октаэдра с некоторыми данными длинами сторон, и ее изометрическое погружение по приведенной схеме дает 8 неконгруэнтных изометричных конфигураций октаэдра.

Недостатком предложенного метода является его неприменимость в случае, когда искомый многогранник окажется изгибаемым. В работе [61] предложен алгоритм, свободный от этого недостатка, но пока реализованный только для многогранников рода 0. Пусть дана развертка из треугольников - будущих граней искомого многогранника, гомеоморфного сфере. Сначала будем предполагать, что комбинаторное строение развертки таково, что в ней нет пустых 3-циклов (их определение было дано в разделе 6). Тогда согласно теореме 1 из [62] в этой развертке существует гамильтонов цикл, т. е. простой цикл, составленный из ребер и проходящий через все вершины развертки. Этот цикл разбивает развертку на две односвязные области, граница каждой из которых содержит все $n$ вершин развертки. Реализуем одну из областей в $\mathbb{R}^{3}$ в виде многогранника с границей - это будет некоторый пространственный $n$-угольник $M$, триангулированный без добавления новых вершин. Он содержит $n-2$ грани, смежные по $n-3$ внутренним ребрам. В [61] доказывается, что многоугольник $M$ может быть построен так, чтобы $n-3$ двугранных углов между согласованно ориентированными гранями были равны заранее заданным значениям $\varphi_{k} \in[0,2 \pi), 1 \leqslant k \leqslant n-3$. Так как все вершины многогранника уже являются вершинами многоугольника $M$, то знание положения $M$ однозначно определяет положение многогранника с теми же вершинами, что и у $M$. Для того чтобы появляющиеся при этом длины ребер - расстояния между соответствующими вершинами - равнялись известным по развертке, но не использованным еще длинам оставшихся $n-3$ ребер, выводятся некоторые уравнения и доказывается, что каждому решению этой системы уравнений соответствует один и только один многогранник, изометрически реализующий в $\mathbb{R}^{3}$ данную развертку (соответственно, если эта система не имеет решений, то реализация невозможна, а если решения составляют непрерывное семейство, то получается изгибаемый многогранник). Построение исходного многоугольника $M$ - конструктивное, уравнения для нахождения значений углов $\varphi_{k}$ - явно выписываемые и линейные относительно $\cos \varphi_{k}$ и $\sin \varphi_{k}, 1 \leqslant k \leqslant n-3$, и поэтому весь алгоритм допускает реализацию на компьютере даже для разверток довольно сложного комбинаторного строения.

\footnotetext{
${ }^{19}$ Кстати, получаем, что число возможных реализаций данной развертки с числом вершин $n$ и топологического рода $g$ в общем случае не превосходит $N \cdot 2 K_{1} \cdot 2 K_{2} \cdots 2 K_{e}$, где $2 N$ - степень многочлена для объема, $2 K_{i}, 1 \leqslant i \leqslant e,-$ степени многочленов (32) для малых диагоналей, а $e=3 n-6+6 g$ - число ребер многогранника; учтено, что при известной длине малой диагонали одну грань к другой можно приклеивать двумя способами. Конечно, на самом деле верхняя оценка должна быть существенно ниже, так как при найденных значениях $n-3$ малых диагоналей для края звезды вершины степени $n>4$ оставшиеся 3 малые диагонали имеют не более чем по два значения.
} 
Если же в развертке есть пустой 3-цикл, то мы можем разрезать многогранник $^{20}$ по этому циклу и разделить развертку на две развертки с треугольными краями. Каждый край мы можем объявить границей двумерного симплекса, сделав новые развертки гомеоморфными сфере. Если хотя бы одна из новых разверток не допускает изометрической реализации в $\mathbb{R}^{3}$, то и исходная развертка не реализуема. Если обе они реализовались как два многогранника в $\mathbb{R}^{3}$, то они будут иметь конгруэнтные грани, соответствующие добавленным двумерным симплексам. Убрав эти грани как открытые множества, склеиваем полученные многогранники по их конгруэнтным треугольным границам и получаем изометрическую реализацию в $\mathbb{R}^{3}$ исходной развертки.

Если в развертке многогранника рода $g \geqslant 1$ с $n$ вершинами найдется гамильтонов цикл, ограничивающий диск, то, реализовав этот диск в $\mathbb{R}^{3}$ как триангулированный пространственный многоугольник, мы снова сможем составить некоторую, на этот раз уже переопределенную, систему линейных уравнений для косинусов и синусов $n-3$ двугранных углов между $n-3$ треугольными гранями в многоугольнике с целью расположения вершин на нужных расстояниях друг от друга. Но для этого алгоритма программной реализации пока нет.

ЗАмЕчАниЕ 5. Между прочим, метод и результат работы [61] дает ответ на вопрос Лежандра о том, каково количество параметров, однозначно определяющих положение многогранника. Он сам дал ответ на этот вопрос в случае выпуклых многогранников (см. [1] и любое последующее издание), показав, что для выпуклых многогранников число таких параметров равно числу сторон многогранника, но в качестве этих параметров нельзя брать длины сторон. В случае общего неизгибаемого, но гомеоморфного сфере многогранника из работы [61] следует, что число параметров тоже равно числу сторон, из них $2 n-3$ - это длины ребер в триангулированном многоугольнике $M$, а $n-3-$ это двугранные углы $\varphi_{k}$ между гранями в $M$.

\section{8. Некоторые алгебро-геометрические свойства многочленов объема}

На наш взгляд, наиболее интригующее в многочлене для объема многогранника заключается в том, что его происхождение (как по смыслу, так и по доказательству) основано на внешней природе многогранника как объекта трехмерного пространства, в то время как сам он определяется только внутренней природой многогранника - его комбинаторным строением и метрикой, и поэтому этот многочлен может быть выписан еще до построения многогранника в пространстве по его натуральной развертке. Это похоже на гауссову кривизну поверхности ${ }^{21}$ - исторически она была определена сначала “внешним" образом как произведение главных кривизн поверхности, а затем было получено ее внутреннее определение через метрику поверхности и появилась большая наука

\footnotetext{
${ }^{20}$ На самом деле нужно говорить не о разрезании многогранника, так как многогранника пока нет, а о разрезании сферы, гомеоморфной телу развертки.

${ }^{21}$ Отметим, однако, что существенное отличие от ситуации с гауссовой кривизной заключается в том, что гауссова кривизна имеет локальное определение, а объем является глобальной характеристикой многогранника.
} 
о связи внутренней и внешней геометрии поверхностей. А вот для многочлена объема пока нет второй части истории гауссовой кривизны - нет внутреннего объяснения происхождения многочлена объема. Возможно, здесь будет найдено некое свойство многих систем алгебраических уравнений, даже более общих, чем система (1), связанных какими-то комбинаторными соотношениями.

18.1. Корни многочлена как объемы многогранника. В этом пункте мы рассмотрим вопрос о том, является ли каждый корень многочлена $Q(V)$ из (6) объемом некоторого реально существующего многогранника с комбинаторным строением и метрикой, породившими этот многочлен ${ }^{22}$. Если такой многогранник существует, будем говорить, что коренъ реализуется.

Этот вопрос на самом деле является очень трудным, и на него пока есть только частичные ответы, имеющие, однако, принципиальное значение. Здесь отправным моментом исследований должно быть задание метрического симплициального комплекса, поэтому мы сначала определим метрические октаэдры Брикара как комбинаторные октаэдры, длины ребер которых удовлетворяют тем же условиям, что и ребра изгибаемых октаэдров Брикара 1-го и 2-го типа. А именно, если в модели октаэдра на рис. 5 длины ребер связаны соотношениями (24), (25), то имеем метрический октаэдр Брикара 1-типа. Если же эти длины связаны соотношениями

$$
\begin{aligned}
& \left|A_{1} B_{1}\right|^{2}=\left|B_{1} A_{2}\right|^{2}=f, \quad\left|A_{2} B_{2}\right|^{2}=\left|A_{2} A_{1}\right|^{2}=e, \quad\left|C_{1} B_{2}\right|^{2}=\left|C_{2} B_{2}\right|^{2}=c, \\
& \left|C_{1} B_{1}\right|^{2}=\left|C_{2} B_{1}\right|^{2}=d, \quad\left|A_{2} C_{1}\right|^{2}=\left|A_{1} C_{2}\right|^{2}=a, \quad\left|A_{1} C_{1}\right|^{2}=\left|A_{2} C_{2}\right|^{2}=b,
\end{aligned}
$$

то имеем метрический октаэдр Брикара 2-го типа. Для этих абстрактно заданных метрических октаэдров многочлен объема выписывается в явном виде. Для 1-го типа он приведен в формуле $(26)$, а для 2-го типа многочлен имеет вид $V^{8}+a_{1}(l) V^{7}+a_{2}(l) V^{6}$. Следовательно, для 1-го типа он имеет два корня, а для 2-го типа - три корня, и в обоих случаях один корень равен 0. В работе [45] доказаны следующие теоремы.

Теорема 6. Если канонический многочлен объема ${ }^{23}$ для данного метрического октаэдра Брикара 1-го типа имеет положительный корень $V_{0}$, то этот метрический октаэдр Брикара допускает изометрическую реализацию в $\mathbb{R}^{3}$ в виде октаэдра, квадрат обгема которого равен $V_{0}$.

Для октаэдров 2-го типа вариантов больше.

ТЕОРема 7. Пусть многочлен обгема для данного метрического октаэдра Брикара 2-го типа имеет положительный корень $V_{1}$. Тогда этот метрический октаэдр допускает изометрическую реализачию в $\mathbb{R}^{3}$ в виде октаэдра, квадрат обгема которого равен $V_{1}$, если выполнено одно из условий:

\footnotetext{
${ }^{22}$ По аналогии с вопросом о нахождении метрики по ее гауссовой кривизне, а затем уж исследовании изометрической погружаемости этой метрики, вопрос можно ставить более широко так: существует ли метрический симплициальный комплекс с данным комбинаторным строением, для которого составленный по нему многочлен $Q(V)$ имеет данный корень, а затем уж спрашивать, является ли этот корень объемом некоторого изометрического погружения найденного симплициального комплекса.

23 Чтобы не уточнять каждый раз связь между переменной $V$ в формуле многочлена и объемом $v$, предположим, что многочлен умножен на такой коэффициент, что $V=v^{2}$.
} 
1) Корень $V_{1}$ имеет кратность 1 ;

2) $V_{1}$ имеет кратность 2 и выполнено условие

$$
(f-d)(e-c) \neq a b .
$$

Заметим, что наличие неравенства (38) и кратность корня $V_{1}$ приводят к равенству нулю некоторой комбинации длин ребер, что, в свою очередь, обеспечивает существование искомого октаэдра.

В случае, когда $(f-d)(e-c)=a b$ и многочлен имеет ненулевой корень $V_{1}$, этот корень обязательно имеет кратность 2 , но оказывается, что его положительность не является достаточным условием реализуемости данного метрического октаэдра, точнее, в [45] приводятся примеры, когда есть реализуемость и когда реализуемости нет. Следовательно, есть случаи, когда не всякий корень многочлена реализуется как объем реально существующего многогранника.

ЗАмЕчАНиЕ 6. Приведенными теоремами вопрос о необходимых или достаточных условиях существования изометрической реализации в $\mathbb{R}^{3}$ метрических октаэдров Брикара 1-го и 2-го типа не исчерпывается, так как остается открытым вопрос о реализации корня $V=0$. Кроме того, и в случае кратного положительного корня какие-либо достаточные условия его реализуемости неизвестны.

Вопрос о реализуемости корней канонического многочлена объема для других многогранников и даже для октаэдров, не являющихся метрическими октаэдрами Брикара, остается пока открытым.

18.2. Корни многочлена объема для изгибаемого многогранника. Второй вопрос, относящийся к корням многочлена объема, - это выяснение особых свойств корней в случае, когда многогранник является изгибаемым. Доказательство существования многочлена объема было дано явным индуктивным построением многочлена (6). Это построение не однозначно, оно зависит от выбора "хорошей" вершины и способа уменьшения ее степени, поэтому для каждого многогранника существуют много многочленов объема. Договоримся называть стандартным любой многочлен объема, построенный по методу первого доказательства основной теоремы 1. Тогда мы можем утверждать справедливость следующей теоремы [63].

Теорема 8. Корень $V^{2}$ любого стандартного многочлена $Q(V)$, соответствующий изгибаемому многограннику, является кратным.

ДокАЗАТЕЛЬСтво. Пусть $P$ - некоторый изгибаемый многогранник с объемом $V=V_{0}$. Значит, есть по крайней мере один изменяющийся двугранный угол, иначе говоря, есть малая диагональ с изменяющейся длиной. Пусть $D-$ одна из таких диагоналей длиной $d$. В наших работах [22] и [21] показано, что перед последним шагом при нахождении желаемого многочлена (6) для объема $V$ и длины $d$ малой диагонали $D$ получаются два уравнения вида

$$
d^{2 K}\left(V^{2 N}+\cdots\right)+d^{2 K-2}\left(b_{1}(l) V^{2 N}+\cdots\right)+\cdots+d^{0}\left(b_{K}(l) V^{2 N}+\cdots\right)=0,
$$


где $b_{i}(l), 1 \leqslant i \leqslant K,-$ некоторые известные многочлены от квадратов длин ребер многогранника, и

$$
C_{0}\left(l, V^{2}\right) d^{2 L}+\cdots+C_{L}\left(l, V^{2}\right) d^{0}=0,
$$

где $C_{L}\left(l, V^{2}\right)=V^{2 M}+c_{1}(l) V^{2 M-2}+\cdots$ и степень многочленов $C_{i}\left(l, V^{2}\right), 0 \leqslant i \leqslant$ $L-1$, относительно $V$ меньше чем $2 M$. Многочлен $Q(V)$ в (6) получается как результант многочленов в левых частях уравнений (39) и (40) при исключении из них переменной $d$. Но в левых частях (39) и (40) для изгибаемого многогранника $P$ все коэффициенты при степенях $d$ равны нулю, иначе эти уравнения давали бы только конечное число значений $d$ в противоречие с предположением о непрерывном изменении длины диагонали $D$. Значит, все элементы в определителе, дающем результант $R$ многочленов в левых частях (39) и $(40)$, в точке $V^{2}=V_{0}^{2}$ равны 0. Вычисление производной $Q_{V^{2}}^{\prime}$ многочлена $Q(V)$ в точке $V_{0}^{2}$ как результанта $R$ дает сумму определителей, в каждом из которых все строки, кроме, быть может, одной (соответствующей очередной дифференцируемой строке), состоят из нулевых элементов, так что $Q_{V^{2}}^{\prime}\left(V_{0}\right)=0$. Следовательно, корень $V_{0}^{2}$ является для $Q(V)$ кратным.

Но приведенное сейчас рассуждение пригодно только для “регулярного” случая нахождения многочлена $Q(V)$ с непосредственным использованием основной леммы 1. При невозможности использования этой леммы, т. е. когда в многограннике нет "хорошей" вершины, нам приходится разрезать многогранник по некоторому пустому 3-циклу, а затем искать многочлены объема для возможно получаемых двух частей многогранника и потом выводить из них многочлен объема для всего многогранника. При этом может оказаться, что одна из частей многогранника не изгибается и для ее многочлена объема мы не сможем применить вышеприведенное рассуждение ${ }^{24}$. Но все же хотя бы одна часть изгибаемого многогранника должна изгибаться, и для ее многочлена объема мы имеем кратность корня, соответствующего объему этой части. А для доказательства кратности корня общего многочлена объема можно использовать следующую простую алгебраическую лемму.

Лемма 7. Пусть дана система трех полиномиальных уравнений

$$
\begin{gathered}
a_{0} x^{n}+a_{1} x^{n-1}+\cdots+a_{n}=0, \quad b_{0} y^{m}+b_{1} y^{m-1}+\cdots+b_{m}=0, \\
z=x+\varepsilon y, \quad \varepsilon= \pm 1 .
\end{gathered}
$$

Пусть $x_{0}$ является кратным корнем первого уравнения, а $y_{0}$ - корень второго уравнения, возможно, простой. Тогда $z_{0}=x_{0}+\varepsilon y_{0}$ является кратным корнем уравнения $q(z)=0$, полученного исключением из системы переменных $x$ и $y$, причем с кратностью не менъшей, чем кратность корня $x_{0}$.

Доказывать эту лемму мы не будем, так как она на самом деле даст нам кратность только корня $V=V_{0}$, а не корня $V_{0}^{2}$, как было выше. Поэтому мы докажем другой вариант леммы.

\footnotetext{
${ }^{24}$ Нужно признать, в работе [63] мы на самом деле не заметили, что этот случай требует отдельного рассмотрения.
} 
ЛЕмма 8. Пусть дана система трех полиномиальных уравнений

$$
\begin{cases}X^{n}+a_{1} X^{n-1}+\cdots+a_{n}=0, & \text { где } X=x^{2}, \\ Y^{m}+b_{1} Y^{m-1}+\cdots+b_{m}=0, & \text { әде } Y=y^{2} \\ Z=z^{2}, \quad z=x+\varepsilon y, \quad \varepsilon= \pm 1 . & \end{cases}
$$

Пусть $X_{0}=x_{0}^{2}$ является кратным корнем первого уравнения, а $Y_{0}=y_{0}^{2}$ корень второго уравнения, возможно, простой. Тогда $Z_{0}$, где $Z_{0}=z_{0}^{2}=$ $\left(x_{0}+\varepsilon y_{0}\right)^{2}$ является кратным корнем уравнения $q(Z)=0, Z=z^{2}$, полученного исключением из системы переменных $X$ и $Y$, причем с кратностью не менъшей, чем кратность корня $X_{0}$.

ДокАзАТЕЛьство. Представим первые два уравнения системы в виде

$$
\left(X-X_{0}\right)^{2} f(X)=\left(x^{2}-x_{0}^{2}\right)^{2} f\left(x^{2}\right)=0, \quad\left(Y-Y_{0}\right) g(Y)=\left(y^{2}-y_{0}^{2}\right) g\left(y^{2}\right)=0
$$

и подставим в первое уравнение $x=z+\varepsilon y$. Получим уравнение

$$
\left[\left(z^{2}+y^{2}-x_{0}^{2}\right)^{2}+4 z^{2} y^{2}\right] f(t)=-4 \varepsilon z y\left(z^{2}+y^{2}-x_{0}^{2}\right) f(t), \quad t=z^{2}+y^{2}+2 \varepsilon z y .
$$

Можем считать, что $f\left(t_{0}\right) \neq 0, t_{0}=\left(z_{0}+\varepsilon y_{0}\right)^{2}$, в противном случае соответствующий корень $X_{0}$ имел бы большую кратность и мы могли бы взять скобку $\left(X-X_{0}\right)$ в большей степени, чем 2 . Возведя обе части уравнения (41) в квадрат, получим

$$
\left[\left(z^{2}+y^{2}-x_{0}^{2}\right)^{2}-4 z^{2} y^{2}\right]^{2} f^{2}(t)=0 .
$$

Полагая $x_{0}=z_{0}+\varepsilon y_{0}$, это уравнение можно представить в виде

$$
\begin{array}{r}
{\left[\left(Z-Z_{0}\right)^{2}-4 \varepsilon z_{0} y_{0}\left(Z-Z_{0}\right)+2\left(y^{2}-y_{0}^{2}\right)\left(Z-Z_{0}\right)-4 y^{2}\left(Z-Z_{0}\right)\right.} \\
\left.-4 Z_{0}\left(y^{2}-y_{0}^{2}\right)-4 \varepsilon\left(y^{2}-y_{0}^{2}\right) z_{0} y_{0}+\left(y^{2}-y_{0}^{2}\right)^{2}\right]^{2} f^{2}(t)=0
\end{array}
$$

откуда следует, что при исключении $y$ из этого уравнения и уравнения системы для $y$ получится уравнение, в котором $\left(Z-Z_{0}\right)^{2}$ является общим множителем всех слагаемых.

Лемма 8 доказана, а из нее следует и теорема, так как метод получения уравнения для $V^{2}$ из многочленов для объемов $V_{1}$ и $V_{2}$ частей многогранника в точности такой же, какой мы сейчас применили для $Z=(x+\varepsilon y)^{2}$ (см., например, начало доказательства основной леммы в разделе 8).

\section{9. Сильная гипотеза кузнечных мехов}

В работе [64] была высказана гипотеза, что изгибаемые многогранники в процессе изгибания не только сохраняют объем, но остаются даже равносоставленными, т.е. в каждой паре из них один многогранник допускает разбиение на конечное число многогранников, которые движениями можно переложить без наложений так, чтобы из них в новом положении составился другой многогранник. Это предположение принято называть сильной гипотезой кузнечных мехов. Вопрос о равносоставленности многогранников с равным объемом составляет содержание третьей проблемы Гильберта. Кратко напомним, что для 
плоскости ответ положителен - два многоугольника равной площади являются равносоставленными, а для пространства это в общем случае неверно - существуют примеры куба и тетраэдра с равными объемами, которые, однако, не являются равносоставленными. Классические результаты подробно изложены в книге [65], а о современном подходе к постановке проблемы и более новых результатах см. [66], [67] и [68].

Известный критерий равносоставленности равновеликих многогранников заключается в равенстве их инвариантов Дена (см. о них [65]) ${ }^{25}$. Однако в случае изгибаемых многогранников в общей постановке проблемы неясно, что понимать под их равносоставленностью как пространственных тел, так как существуют не вложенные и даже не погруженные изгибаемые полиэдральные поверхности, по отношению к которым нельзя говорить об ограниченном ими теле. Между тем определение инвариантов Дена не требует привлечения тела, ограниченного данной многогранной поверхностью, поэтому задачу ставят так: имеют ли наложимые друг на друга изгибанием многогранники (понимаемые как многогранные поверхности) совпадающие инварианты Дена? Так как совпадение инвариантов Дена для каждой пары многогранников означает, что инварианты Дена для каждого многогранника из семейства изгибаний должны совпадать с инвариантами Дена многогранника, выбранного в качестве исходного, то для справедливости сильной гипотезы кузнечных мехов инварианты Дена должны быть постоянными в ходе изгибания. Ясно, что в случае вложенности многогранников в семействе изгибаний постоянство инвариантов Дена даст и факт их равносоставленности в силу неизменности объема изгибаемого многогранника.

В работе [69] на вопрос о постоянстве инвариантов Дена дан положительный ответ для изгибаемых октаэдров всех трех типов, а также и для изгибаний многогранника Штеффена, однако уже в случае изгибаемых подвесок с 6-звенным экватором в работе [70] показано, что в ходе изгибания могут появиться многогранники, у которых существуют инварианты Дена, не совпадающие с соответствующими инвариантами исходного многогранника. Но стоит обратить внимание на то, что изгибаемые подвески не являются вложенными многогранниками, поэтому вопрос о равносоставленности вложенных изгибаемых многогранников остается открытым.

\section{0. Еще один инвариант изгибаемых многогранников}

Существует еще одна геометрическая характеристика многогранников, инвариантная при изгибаниях, это - интегральная средняя кривизна $H$ ориентируемого вложенного многогранника, определяемая по формуле

$$
H=\frac{1}{2} \sum l_{i}\left(\pi-\alpha_{i}\right)
$$

\footnotetext{
${ }^{25}$ Наиболее геометрической формулировкой этого признака в части его необходимости является следующее условие Брикара: если два многогранника $A$ и $B$ равносоставлены, то существуют натуральные числа $n_{j}$ и $n_{i}^{\prime}$ и целое $p$ такие, что

$$
n_{1} \alpha_{1}+\cdots+n_{q} \alpha_{q}=n_{1}^{\prime} \beta_{1}+\cdots+n_{r}^{\prime} \beta_{r}+p \pi,
$$

где $\alpha_{1}, \ldots, \alpha_{q}$ и $\beta_{1}, \ldots, \beta_{r}$ - величины двугранных углов в многогранниках $A$ и $B$ соответственно.
} 
где суммирование идет по всем ребрам, $l_{i}$ - длина $i$-го ребра, $\alpha_{i}$ - двугранный угол при $i$-м ребре, $1 \leqslant i \leqslant e, e-$ число ребер. Считаем, что мы имеем дело с многогранником, ориентированным нормалью к какой-нибудь грани с распространением направления нормали на другие грани по стандартному правилу согласования ориентации граней. Тогда двугранный угол $\alpha_{i}$ при ребре с номером $i$ считается как геометрический угол изнутри многогранника. В работе [71] доказано, что если вложенный ориентируемый многогранник изгибаемый, то его интегральная средняя кривизна инвариантна при изгибаниях. Более того, это свойство инвариантности $H$ верно и в многомерном случае $\left(\right.$ в $\mathbb{R}^{n}, n \geqslant 4$, в формуле (42) вместо длины ребра надо брать $(n-2)$-мерный объем $(n-2)$-мерной грани, общей для двух $(n-1)$-мерных граней, с соответствующим пониманием ориентации и угла $\left.\alpha_{i}\right)$.

Доказательство этого утверждения простое, и мы его приведем. Пусть $K(t)-$ гладкое (по параметру $t$ ) семейство 3 -мерных симплексов в $E^{3}$. Обозначим через $L(t)$ некоторое ребро в $K(t)$, и пусть $F_{1}(t)$ и $F_{2}(t)$ - прилегающие к нему грани. Возьмем на ребре $L(t)$ произвольную точку $M(t)$ и построим в ней 4 вектора - внешние нормали $\mathbf{N}_{1}$ и $\mathbf{N}_{2}$ к $K(t)$, перпендикулярные соответственно к граням $F_{1}$ и $F_{2}$, и внешние по отношению к ребру $L(t)$ нормали $\mathbf{v}_{1}$ и $\mathbf{v}_{2}$ на гранях $F_{1}$ и $F_{2}$ в соответствии с ориентацией этих граней (рис. 11 ).

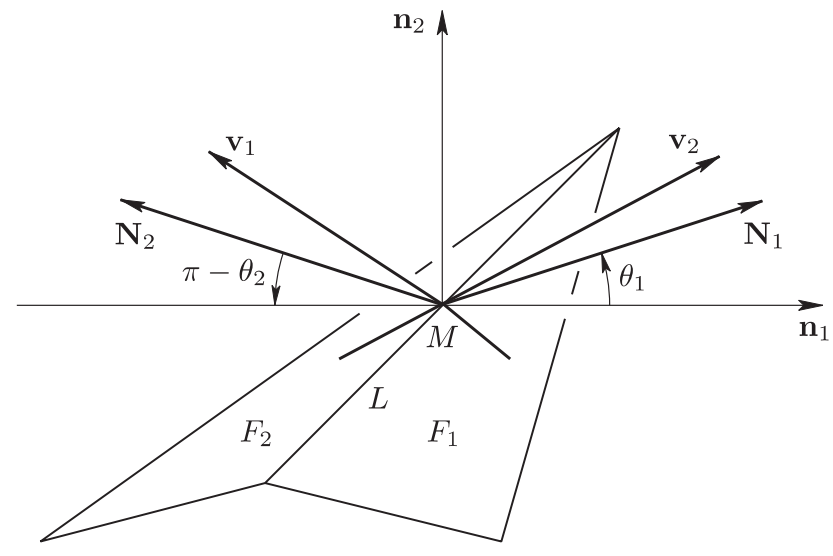

Рис. 11

Нормали $\mathbf{N}_{1}$ и $\mathbf{N}_{2}$ мы выберем единичные, а нормали $\mathbf{v}_{1}$ и $\mathbf{v}_{2}$ выберем с длиной, равной $l(t)$ - длине ребра $L(t)$. На ортогональной к $L(t)$ плоскости $T$ введем некоторую декартову систему координат с единичными ортами $\mathbf{n}_{1}$ и $\mathbf{n}_{2}$. Тогда

$$
\begin{aligned}
\mathbf{N}_{1}(t) & =\cos \theta_{1} \mathbf{n}_{1}+\sin \theta_{1} \mathbf{n}_{2} \\
\mathbf{N}_{2}(t) & =\cos \theta_{2} \mathbf{n}_{1}+\sin \theta_{2} \mathbf{n}_{2}, \\
\mathbf{v}_{1}(t) & =l(t)\left(-\sin \theta_{1} \mathbf{n}_{1}+\cos \theta_{1} \mathbf{n}_{2}\right), \\
\mathbf{v}_{2}(t) & =l(t)\left(\sin \theta_{2} \mathbf{n}_{1}-\cos \theta_{2} \mathbf{n}_{2}\right),
\end{aligned}
$$

где $\theta_{i}$ - стандартно ориентированные углы соответственно между $\mathbf{n}_{1}$ и нормалями $\mathbf{N}_{i}, i=1,2$. Легко видеть, что внутренний двугранный угол $\alpha(t)$ между 
плоскостями граней $F_{1}$ и $F_{2}$ равен $\pi-\left(\theta_{2}(t)-\theta_{1}(t)\right)$. Мы хотим вычислить изменение угла $\alpha(t)$ в ходе гладкой деформации ${ }^{26}$ с параметром $t$. Добавляя движение, можем считать, что плоскость $T$ и введенные на ней векторы $\mathbf{n}_{1}$ и $\mathbf{n}_{2}$ в ходе деформации остаются постоянными. Имеем

$$
\begin{aligned}
& \frac{d \mathbf{N}_{1}}{d t}=-\left(\sin \theta_{1} \mathbf{n}_{1}-\cos \theta_{1} \mathbf{n}_{2}\right) \theta_{1}^{\prime}(t), \\
& \frac{d \mathbf{N}_{2}}{d t}=-\left(\sin \theta_{2} \mathbf{n}_{1}-\cos \theta_{2} \mathbf{n}_{2}\right) \theta_{2}^{\prime}(t),
\end{aligned}
$$

откуда получаем нужное нам уравнение

$$
l(t) \frac{d \alpha(t)}{d t}=\left(\frac{d \mathbf{N}_{1}}{d t}, \mathbf{v}_{1}\right)+\left(\frac{d \mathbf{N}_{2}}{d t}, \mathbf{v}_{2}\right)=-\left(\mathbf{N}_{1}, \frac{d \mathbf{v}_{1}}{d t}\right)-\left(\mathbf{N}_{2}, \frac{d \mathbf{v}_{2}}{d t}\right) .
$$

Рассмотрим теперь многогранную поверхность $P$ с невырожденными треугольными гранями (т. е. не нужно требовать ни вложенности, ни даже погруженности). Предположим, что она изгибается, и применим формулу (43) к двугранным углам при всех ребрах. Получим

$$
\sum_{k} l_{k}\left(\frac{d \alpha_{k}(t)}{d t}\right)=-\sum_{i}\left[\sum_{j}\left(\mathbf{N}_{i}, \frac{d \mathbf{v}_{i j}}{d t}\right)\right]
$$

где индекс $k$ соответствует номерам ребер, индекс $i$ - номерам граней, а $j$ изменяется от 1 до 3 на каждой грани. Векторы $\mathbf{v}_{i j}$ являются внешними нормалями к ребрам грани с номером $i$ и имеют модуль, равный длине стороны с номером $j=1,2$ или 3. По известной формуле Минковского $\sum_{j=1}^{3} \mathbf{v}_{i j}=0$ при каждом $i$, следовательно, в итоге вся сумма в левой части уравнения равняется нулю, что и означает равенство нулю производной средней кривизны $H$ многогранника при его изгибании. Более того, это свойство инвариантности $H$ верно и в многомерном случае.

ЗАмЕчАниЕ 7. Из доказанной формулы и из определения (42) средней кривизны следует, что при деформациях многогранника дифференциал его средней кривизны линейно зависит от дифференциалов длин ребер:

$$
\left.d H=-\frac{1}{2} \sum_{i} \alpha_{i} d l_{i} \quad \text { (индекс } i \text { изменяется по ребрам }\right) .
$$

В [72] проведен интересный сравнительный анализ способов доказательства инвариантности объема и интегральной средней кривизны многогранников. Автор замечает, что у свойства инвариантности объема нет инфинитезимального аналога, т. е. если многогранник допускает нетривиальное бесконечно малое изгибание 1-го порядка, то соответствующая первая вариация объема может быть и ненулевой (но если бесконечно малое изгибание получено как поле скоростей изгибания, то вариация объема, конечно, равна нулю). Косвенно это

\footnotetext{
${ }^{26}$ На самом деле можно было бы рассматривать не тетраэдр, а два треугольника с общим ребром и двугранный угол при этом ребре.
} 
означает, что мы не можем связать дифференциал объема с дифференциалами длин ребер, и этим объясняется, что оба известных доказательства инвариантности объема многогранника при его изгибании основаны на алгебраических методах ${ }^{27}$. Между тем, дифференциал средней кривизны, как это видно из вышеприведенного замечания, линейно связан с дифференциалами длин ребер. Покажем, следуя работе [72], что между средней кривизной и длинами ребер многогранника нет алгебраической зависимости.

Рассмотрим тетраэдр на рис. 1. Элементарными вычислениями находим двугранный угол $\varphi$ при ребре $l_{1}$ :

$$
\cos \varphi=\frac{4 S_{123}^{2}+4 S_{146}^{2}-l_{1}^{2} l_{5}^{2}+\left[\left(l_{3}^{2}+l_{4}^{2}\right)-\left(l_{2}^{2}+l_{6}^{2}\right)\right]^{2}}{8 S_{123} S_{146}},
$$

где $S$ с индексами - площади прилегающих к ребру $l_{1}$ граней, а в остальных слагаемых участвуют квадраты длин попарно скрещивающихся ребер. Пусть у тетраэдра в основании лежит правильный треугольник с основанием 1 , а боковые ребра имеют одинаковую длину $l$. Тогда формула (45) даст следующие значения для косинусов двугранных углов:

$$
\cos \varphi=\frac{1}{\sqrt{3} \sqrt{4 l^{2}-1}}, \quad \cos \psi=\frac{2 l^{2}-1}{4 l^{2}-1}
$$

( $\varphi$ - двугранные углы при основаниях, а $\psi$ - двугранные углы при боковых ребрах). Значит, средняя кривизна тетраэдра равна

$$
H=\frac{3}{2}\left(\pi-\arccos \frac{1}{\sqrt{3} \sqrt{4 l^{2}-1}}\right)+\frac{3}{2} l\left(\pi-\arccos \frac{2 l^{2}-1}{4 l^{2}-1}\right) .
$$

Из этой формулы усматриваем, что $H$ не может быть алгебраической функцией длин ребер тетраэдра, так как алгебраическая функция может иметь точки ветвления только конечного порядка, а обратные тригонометрические функции, продолженные на комплексную плоскость, имеют ветвления бесконечного порядка. О доказательстве неалгебраичности средней кривизны для многогранников произвольного комбинаторного строения см. работу [72], результат которой можно сформулировать так: для многогранников любого комбинаторного строения можно найти такую их конфигурачию, для которой средняя кривизна не является алгебрачческой функиией длин ребер.

Вернемся к общей формуле (42) для средней кривизны многогранника. Если мы знаем значения малых диагоналей, то по формуле (45) можем найти значения косинусов двугранных углов (в формуле (42) надо подставить вместо $l_{5}$ соответствующую малую диагональ $d$, остальные величины известны по значениям длин ребер), а по ним найдутся два значения двугранных углов вида $\varphi$ и $2 \pi-\varphi$, из которых следует выбрать нужное значение как соответствующее

\footnotetext{
${ }^{27}$ Но в период поиска доказательства или опровержения гипотезы кузнечных мехов одним из возможных подходов к ее исследованию было изучение поведения объема нежесткого многогранника при его бесконечно малом изгибании 1-го порядка (см. [16]). Хотя этот подход не дает опровержения гипотезы, но он помогает отобрать среди бесконечно малых изгибаний нежесткого многогранника те, которые продолжимы в его “настоящие" изгибания.
} 
внутренним сторонам граней. В итоге мы получаем точную формулу для выражения средней кривизны через длины ребер и малых диагоналей:

$$
H=\frac{1}{2} \sum_{i} l_{i}\left(\pi-\arccos f_{i}(l, d)\right) \quad \text { (суммирование по ребрам), }
$$

в которой $l$ и $d$ обозначают совокупность квадратов длин ребер и малых диагоналей многогранника, а функции $f_{i}$ определяются по формуле (45) с соответствующими значениями длин ребер и малых диагоналей (в каждой функции $f_{i}$ участвуют 5 ребер и одна малая диагональ). Формула (46) пригодна для всех конфигураций многогранников с данным комбинаторным строением и данными длинами ребер. Мы знаем уравнения для каждой малой диагонали, но, во-первых, не знаем согласованных решений всей совокупности этих уравнений, во-вторых, для некоторых из них, или даже для всех, их коэффициенты могут равняться нулю, и тогда мы не сможем найти малые диагонали. Следовательно, стоит задача найти такое уравнение, из которого можно было бы получить все априори возможные значения средней кривизны. Результат работы [72] говорит, что такое уравнение не может быть алгебраическим, значит, его надо искать среди аналитических или дифференциальных уравнений.

По поводу возможности нахождения последних можно привести следующие соображения. Функция $H$ является однородной с порядком однородности 1 (действительно, все малые диагонали имеют линейную размерность 1 и поэтому аргументы у arccos являются однородными порядка 0). Тогда имеем равенство

$$
\sum_{i} l_{i} \frac{\partial H}{\partial l_{i}}=H
$$

причем

$$
\frac{\partial H}{\partial l_{i}}=\frac{1}{2}\left[\left(\pi-\arccos f_{i}(l, d)\right)+l_{i} \frac{1}{\sqrt{1-f_{i}^{2}}} \frac{\partial f_{i}}{\partial l_{i}}+\sum_{j \neq i} l_{j} \frac{1}{\sqrt{1-f_{j}^{2}}} \frac{\partial f_{j}}{\partial l_{i}}\right] .
$$

Отсюда видно, что в общем положении ${ }^{28}$ все производные второго порядка функции $H$ являются алгебраическими функциями от длин ребер, что дает надежду показать, что существует прямой способ вычисления ${ }^{29}$ априорных значений средней кривизны еще до построения многогранника по его метрике и комбинаторному строению, как это мы можем сделать для объема.

\section{1. Многогранники в сферическом и гиперболическом пространствах}

Среди работ, имеющих прямое отношение к основной теме статьи, мы упомянем прежде всего работу [73]. В ней строится пример изгибаемого многогранника (октаэдра), расположенного строго внутри 3-мерной полусферы, объем

\footnotetext{
${ }^{28}$ Имеется в виду, что производные малых диагоналей по $l_{i}$ заведомо нельзя определить, например, для изгибаемых многогранников.

${ }^{29}$ Т. е. не обращаясь к вычислению малых диагоналей, что, как мы знаем, не легко и, самое главное, не всегда возможно.
} 
которого изменяется в процессе изгибания. И так как дифференциалы объема и средней кривизны в пространствах $M_{n}$ ненулевой постоянной кривизны $K$ связаны между собой формулой Шлефли (см., например, [71])

$$
K d V_{n}=-\frac{1}{2(n-1)} \sum_{j} V_{n-2}^{(j)} d \beta_{j}
$$

где $n$ - размерность пространства, $V_{n}$ есть $n$-мерный объем тела в $M_{n}$, ограниченного деформируемым многогранником $P$, а $\beta_{j}$ - угол между двумя $(n-1)$ мерными гипергранями многогранника, примыкающими к общей $(n-2)$-мерной грани, имеющей в некоторой нумерации номер $j$ и $(n-2)$-мерный объем $V_{n-2}^{(j)}$, то при изгибании многогранника с изменяющимся объемом его средняя кривизна тоже не остается постоянной. Таким образом, в сферическом пространстве обе величины - объем и средняя кривизна, - инвариантные в $\mathbb{R}^{3}$ при изгибании, не являются таковыми в сферическом пространстве. Про поведение этих характеристик для изгибаемых многогранников в пространстве Лобачевского ничего не известно, лишь относительно объема можно утверждать, что дифференциал объема не является линейной функцией от дифференциалов длин ребер, что показывается построенным в работе [74] примером.

Еще одним примером сопоставления и продолжения задач и результатов метрической теории многогранников в $\mathbb{R}^{3}$ в сферическое или гиперболическое пространство является работа [75], в которой, по аналогии с идеями статьи [43], в предположении некоторой метрической симметрии октаэдра удается найти формулы для его объема в функции длин ребер. Однако принципиальная разница в результатах этих работ состоит в том, что в [43] предлагается формула, позволяющая вычислять объемы всех октаэдров, изометричных октаэдру с данной симметрией, а в [75] дается точная формула объема только для самого октаэдра с данной симметрией, а вопрос об объемах октаэдров, изометричных данному, но не имеющих пространственной симметрии в своей конфигурации, остается открытым.

Отправным пунктом идеи доказательства теоремы 1 была формула (4) для объема тетраэдра в функции длин его ребер. В сферическом и гиперболическом пространствах для объема тетраэдра такой простой формулы нет ${ }^{30}$. Во многих работах предлагаются различные аналитические формулы (см., например, [78]-[81]), однако среди них нет формулы, которая была бы общепризнана как наиболее простая. Но дело даже не в простоте или сложности этих формул, дело в невозможности использования их для индуктивного перехода от более сложных многогранников к менее сложным. К тому же в сферическом и гиперболическом пространствах нет подобия, поэтому в формулах для объема исчезает однородность относительно длин ребер. Отсюда как следствие получается, что пока основная масса работ направлена на получение

\footnotetext{
30 Даже для площади треугольника аналог формулы Герона на плоскости Лобачевского кривизны $K=-1$ имеет устрашающе сложный вид ([76; гл. V, задача 31.1] и [77; с. 36]):

$\sin \frac{\Delta}{2}=\frac{\left[\left(e^{2 \sigma}-1\right)\left(e^{2 \sigma-2 \alpha}-1\right)\left(e^{2 \sigma-2 \beta}-1\right)\left(e^{2 \sigma-2 \gamma}-1\right)\right]^{2}}{\left(e^{\alpha}+1\right)\left(e^{\beta}+1\right)\left(e^{\gamma}+1\right)}=\frac{\sqrt{\operatorname{sh} \sigma \operatorname{sh}(\sigma-\alpha) \operatorname{sh}(\sigma-\beta) \operatorname{sh}(\sigma-\gamma)}}{2 \operatorname{ch}(\alpha / 2) \operatorname{ch}(\beta / 2) \operatorname{ch}(\gamma / 2)}$,
}

где $\Delta$ - площадь треугольника со сторонами длины $\alpha, \beta, \gamma$, a $2 \sigma$ - периметр треугольника. 
формул для объемов конкретных многогранников сочетанием различных алгебраических, аналитических, синтетических и комбинаторно-топологических методов (см., например, [82]-[84]). Хороший краткий обзор таких работ дан в [85], а общие вопросы комбинаторно-метрической геометрии многогранников в пространстве Лобачевского вместе с результатами об объемах некоторых конкретных многогранников рассмотрены в [86]. Между тем вопрос о поведении объема многогранника в сферическом или гиперболическом пространстве при его изгибании содержателен, так как в обоих пространствах существуют изгибаемые многогранники. Для сферического пространства это установлено в [73], а для гиперболического пространства - в работе [87], в которой доказано, что изгибаемые октаэдры в гиперболическом пространстве содержат по крайней мере те же три типа, что найдены Брикаром в евклидовом случае. Но подходов к нахождению общей формулы объема для семейства изометричных многогранников не видно. Более того, есть некоторые отрицательные, так сказать, "показания" к существованию такой формулы. А именно, известно, что тетраэдр с двугранными углами $A, B, C, D, E, F$ однозначно определяется своей матрицей Грама

$$
G=\left(\begin{array}{cccc}
1 & -\cos A & -\cos B & -\cos F \\
-\cos A & 1 & -\cos C & -\cos E \\
-\cos B & -\cos C & 1 & -\cos D \\
-\cos F & -\cos E & -\cos D & 1
\end{array}\right)
$$

(каждая строка соответствует углам при одной вершине). Гипотеза Зейделя (1986) состояла в том, что объем идеального гиперболического тетраэдра можно выразить как функцию от определителя и перманента его матрицы Грама, а усиленная гипотеза Грама, выдвинутая И. Ривиным и Ф. Лю, утверждала, что объем любого неевклидова тетраэдра является функцией только от определителя его матрицы Грама. В работах [88] и [89] показано, что обе гипотезы неверны (но при этом установлено, что в каждом из классов остроугольных или тупоугольных идеальных тетраэдров исходная гипотеза Зейделя справедлива).

Мы видим, что так как найти для изометричных многогранников в гиперболическом пространстве многочлен или иную функцию объема мало надежд, то, может быть, там удастся доказать гипотезу кузнечных мехов не как следствие какого-либо аналога теоремы 1 , а как самостоятельное утверждение. Что касается сферического пространства, то автор примера изгибаемого октаэдра с переменным объемом В. А. Александров высказывал мнение, что его пример в некотором смысле является случайным, а для "большинства" изгибаемых многогранников в сферическом пространстве их объем должен оставаться постоянным.

В последние годы появился ряд работ, в которых узлам и зацеплениям сопоставляют объемы некоторых искусственных образований, которые условно называются виртуалъными многогранниками (см. [90]-[92]), но многие результаты еще не опубликованы. Такие образования можно строить в любой геометрии постоянной кривизны, и они, как тела, имеют границы, получаемые отождествлением некоторых поверхностей, порождаемых отдельными участками узлов и зацеплений. Если это все происходит в евклидовой геометрии, то доказывается, что тогда объемы таких виртуальных многогранников являются корнями 
некоторых многочленов, как в теореме 1. В связи с этими работами можно поставить вопрос о нахождении объемов многогранников, заданных в локально евклидовых или локально гиперболических пространствах, в которых изометрические прообразы этих многогранников являются замкнутыми и без отождествления граней. Проиллюстрируем эту идею частично следующим примером. Пусть на евклидовой плоскости с координатами $(x, y)$ задан прямоугольник П: $0 \leqslant x \leqslant a, 0 \leqslant y \leqslant b$ и из него отождествлением сторон $x=0$ и $x=a$ получается многообразие-кольцо $P$ с краем и с локально евклидовой метрикой. Мы можем вычислить площадь этого многообразия $P$ и в то же время можем рассмотреть на другой плоскости $T$ с координатами $(u, v)$ кольцо $K: 0<r^{2}<$ $u^{2}+v^{2}<R^{2}$ с локально евклидовой метрикой $d s^{2}=\left(u^{2}+v^{2}\right)\left(d u^{2}+d v^{2}\right)$, в котором (при соответствующим образом подобранных $r$ и $R$ ) изометричная $P$ область получается как кольцо уже без операции отождествления, т. е. путем введения плоскости $T$ с локально евклидовой метрикой мы избавились от необходимости использования такой абстрактной операции, как отождествление сторон (или граней) и можем работать с объектом, наглядный образ которого реально находится во взаимно однозначном соответствии с самим объектом. Поэтому можно ставить вопрос об объеме многогранников в пространствах, локально являющихся пространствами постоянной кривизны, и таким образом расширить класс многогранников, на которые будут распространяться теоремы, доказываемые для многогранников в пространствах, имеющих в целом стандартную метрику постоянной кривизны.

Формула Шлефли (47), очень полезная в неевклидовых пространствах постоянной кривизны, может оказаться полезной и в евклидовом случае. Для этого надо исследовать ее следствия в евклидовом пространстве, рассматривая его как предельный случай пространств кривизны $K<0$ и $K>0$ при стремлении $|K|$ к нулю. Частично это сделано в [71; с. 676], где показано, что формула для дифференциала средней кривизны многогранника в евклидовом пространстве может быть получена как предел формулы Шлефли в сферическом пространстве при неограниченном возрастании радиуса $R$ сферы. Этот результат получается при рассмотрении главных членов 1-го порядка малости относительно $1 / R^{2} \rightarrow 0$, но остается открытым вопрос о влиянии членов 3-го, 4-го и т. д. порядков относительно $1 / R \rightarrow 0$.

\section{2. Многогранники в многомерных пространствах}

Про изгибаемость многогранников в пространствах размерности 4 и выше известно очень мало, еще меньше известно про их объемы. Есть работа [93], в которой описываются изгибаемые кросс-политопы в $E^{4}$. Эти многогранники определены в любом $E^{n}, n>3$, как аналоги октаэдров: они имеют $2 n$ вершин, разбитых на пары $\left(p_{1}^{i}, p_{2}^{i}\right), 1 \leqslant i \leqslant n$, их ребра в количестве $C_{n}^{2}=2 n(n-1)$ соединяют вершины $p_{j_{1}}^{i}$ и $p_{j_{2}}^{k}$, где $i \neq k$ и $j_{1}, j_{2} \in\{1,2\}$; гипергранями являются $2^{n}$ симплексов с вершинами $p_{j_{1}}^{1}, p_{j_{2}}^{2}, \ldots, p_{j_{n}}^{n}, j_{1}, j_{2}, \ldots, j_{n} \in\{1,2\}$. Комбинаторную модель кросс-политопов наглядно можно представить так: в $E^{n}$ вводится система координат $O x_{1} \ldots x_{n}$, на каждой из осей координат берется пара симметричных относительно начала координат точек и в каждом гипероктанте из на- 
ходящихся там вершин строится $(n-1)$-мерный симплекс. Реализации в пространстве такой модели могут иметь разные формы, в том числе и невыпуклые. Вот среди таких невыпуклых и даже имеющих самопересечения многогранников А. Вальц (Waltz) нашла примеры изгибаемых кросс-политопов, и на их основе в [93] построен более общий класс изгибаемых кросс-политопов, причем этот метод можно использовать для построения изгибаемых кросс-политопов в 4-мерных эллиптическом и гиперболическом пространствах. Что касается более высоких размерностей, то известны только некоторые характеристики нежестких (т. е. допускающих нетривиальные бесконечно малые изгибания 1-го порядка) кросс-политопов (см. [94]).

Для объемов многогранников в $E^{n}, n>3$, можно применить предложенный в разделе 16 метод вычисления их объема с использованием длин диагоналей и тем самым установить некоторую алгебраическую связь объема с длинами ребер и диагоналей, а потом попытаться исключить длины диагоналей с помощью различных уравнений связи для длин ребер в полном графе, однако этот путь еще не реализован (для его применения могут оказаться полезными теоремы о рангах матрицы расстояний [95] и матрицы Кэли-Менгера, [33], [96]).

Есть еще один способ установления связи между объемом и длинами ребер и диагоналей. По приведенному в разделе 2 определению обобщенного объема симплициального многогранника, он равен сумме объемов тетраэдров, построенных на согласованно ориентированных гипергранях многогранника с общей вершиной в произвольно выбранной точке $O$. Выберем в качестве $O$ одну из вершин многогранника. Тогда квадрат объема каждого тетраэдра будет равен определителю Кэли-Менгера, элементы которого даются как квадраты длин ребер основания тетраэдра (т.е. некоторой гиперграни многогранника) и квадраты длин диагоналей, идущих от выбранной вершины многогранника до вершин соответствующей гиперграни (с постоянным коэффициентом, зависящим лишь от $n$, и со знаком, определяемым ориентацией грани). Следовательно, для объема $V$ многогранника имеем формулу

$$
V=\varepsilon_{k} \sqrt{V_{k}^{2}}, \quad \varepsilon= \pm 1,
$$

где индекс $k$ принимает значения номеров всех гиперграней, а $V_{k}^{2}$ являются многочленами от квадратов длин ребер и диагоналей, идущих от выбранной вершины до остальных вершин многогранника. Многократным возведением в квадрат с соответствующей группировкой членов уравнений можно придти к уравнению вида

$$
V^{2 N}+a_{1}\left(l^{2}, d^{2}\right) V^{2 N-2}+\cdots+a_{N}\left(l^{2}, d^{2}\right)=0,
$$

где коэффициенты $a_{j}, 1 \leqslant j \leqslant N$, являются многочленами от квадратов длин ребер (с общим обозначением $l^{2}$ ) и квадратов длин диагоналей (с общим обозначением $d^{2}$ ). Этот способ дает нам возможность получить аналог теоремы 1 и, как следствие, доказать гипотезу кузнечных мехов в $E^{n}$ для одного класса многогранников, которые мы называем пирамидами. Многогранники с таким названием были впервые введены в рассмотрение, по-видимому, в [97] и определяются они как многогранники, у которых есть по крайней мере одна вершина, соединенная ребрами со всеми остальными вершинами. Такие 
симплициальные многогранники в $E^{3}$ существуют во всех топологических родах, как ориентируемых, так и неориентируемых [29], а в произвольном $E^{n}$ их можно построить над любой полиэдральной гиперповерхностью с краем, у которой все вершины расположены на крае: для этого достаточно взять любую точку пространства и, соединив ее ребрами с вершинами края, "закрыть" край добавлением $(n-1)$-мерных симплексов, построенных на этих ребрах и $(n-2)$-мерных симплексах края. Для ориентируемого симплициального многогранника-пирамиды объем можно вычислить, взяв за общую вершину $n$-мерных тетраэдров ту вершину многогранника, которая соединена со всеми другими его вершинами. Тогда в формуле (48) коэффициенты $a_{j}$ будут зависеть только от длин ребер и мы получим аналог теоремы 1 в любой размерности. Из этой конструкции получаем такое следствие.

СлЕДСТВиЕ 8. Если в пространстве $E^{n}, n>3$, в многограннике с $N$ вершинами число диагоналей менъше $N / 2$, то для обгемов таких многогранников существует многочлен обгема и для них верна гипотеза кузнечных мехов.

\section{3. Некоторые нерешенные проблемы}

В заключение приведем далеко не полный перечень проблем, которые еще ждут своего решения, сопроводив их некоторыми комментариями. Заметим, что по ходу изложения материала уже было сформулировано около десятка нерешенных вопросов, которые мы старались выделить курсивом или жирным шрифтом (но не всюду это получилось).

1. Доказать или опровергнуть гипотезу кузнечных мехов для многогранников в пространстве Лобачевского. Здесь трудно надеяться на получение полиномиального аналога формулы Герона, но, может быть, удастся доказать, что объем является нулем некоторой аналитической функции (явной или являющейся решением некоторого дифференциального уравнения), что тоже будет достаточным для справедливости гипотезы кузнечных мехов.

2. Доказать гипотезу кузнечных мехов в многомерном евклидовом пространстве. Проблема здесь в том, что для классификации комбинаторного строения многоугольников нет целочисленных параметров типа числа вершин и топологического рода, и поэтому неизвестно, как применять рассуждение по индукции. И к тому же самые простые многогранники, например подвески в $\mathbb{R}^{4}$ над двумерным тороидальным многогранником из $\mathbb{R}^{3}$, не являются многообразием, а относятся к псевдомногообразиям. Для начала надо хотя бы установить наличие алгебраической связи между объемом и длинами ребер многогранника, идея нахождения которой описана во втором абзаце раздела 22.

3. Рассмотрение многогранников как в разделах 16 и 22 в дополнительном предположении, что известны длины всех их диагоналей, приводит к общему вопросу описания строения множества $N$ точек с известными $N(N-1) / 2$ расстояниями во всех парах точек, или описания конфигурации полного графа с прямолинейными ребрами в евклидовом пространстве $\mathbb{R}^{n}, n<N$, с известными длинами ребер. Дело в том, что даже в простейшей задаче описания тетраэдров (т. е. при значениях $n=3, N=4$ ) с заданным набором длин из 6 чисел без предварительного их распределения по ребрам, поставленной в свое 
время автором студенту МГУ Н. Ермилову, оказалось, что в общем случае существует 30 (!) неконгруэнтных конфигураций множества из 4 точек (их можно отождествлять с соответствующими тетраэдрами), имеющих один и тот же набор расстояний между точками [98], [99]. А задача описания конфигураций множества точек с известными расстояниями между всеми точками очень важна во многих областях и техники, и естествознания. Например, в физике во многих задачах ищется минимум какого-либо функционала (обычно называемого потенциалом или энергией), зависящего от расстояний между всеми точками рассматриваемой системы (атомы, молекулы, тела). Математически в этих функционалах (например, в потенциале Леннард-Джонса) участвуют только сами расстояния, и после нахождения значений этих расстояний, дающих минимум, надо будет еще установить геометрическую структуру того множества, на котором достигается этот минимум. Таким образом, появляется задача описания конфигураций множеств $N$ точек с заданным набором расстояний между ними с возможным выделением среди них многогранников с заданными длинами ребер. Среди этих задач выделяются задачи с малым числом различных расстояний (например, в той же задаче с 4 вершинами есть только одно решение, если все расстояния равны, есть не более трех решений, если различных расстояний всего 2, и т. д.). Некоторые результаты в такой постановке см. в [100]. Об оценках числа различных неизгибаемых (т. е. локально однозначно определенных) конфигураций графов с данными длинами ребер в пространствах постоянной кривизны и произвольной размерности см. [101].

4. Описанную сейчас задачу можно связать со следующей задачей. Выше в разделе 16 было отмечено, что, имея одномерный каркас тора с 7 вершинами, можно "обшить" этот каркас 14 треугольниками 120 способами так, что получатся 120 неконгруэнтных (и не изометричных) многогранников рода 0. Поставим аналогичный вопрос в общем случае: пусть в данном многограннике удалены все грани (как открытые множества) и все ребра остались на месте, но мы не запомнили, какие ребра были на каких гранях. Спрашивается, если мы будем пытаться восстановить грани (с соблюдением стандартного условия, что к каждому ребру примыкают ровно две грани и две грани могут встречаться или только в вершине или по целому ребру), то обязательно ли получим исходный многогранник? Случаи положительных ответов на этот вопрос рассмотрены в работах [102], [103], но есть и случаи неединственности (см., например, [56], [104], [105]). Каков алгоритм восстановления многогранника по его одномерному каркасу (или, по-другому, по его 1-скелету) и как получить многогранник с экстремальными значениями объема или суммарной площади граней?

5. Что назвать "каноническим" многочленом объема для многогранников рода $g>0$ ? Если многочлен объема (общее определение многочлена объема см. в разделах 5 и 12) - наименьшей степени, то является ли он единственным и не может ли он быть разным для данного фиксированного комбинаторного строения в зависимости от значений длин ребер? Дать корректное определение канонического многочлена объема и указать алгоритм его вычисления.

6. Доказать, что объем изгибаемого многогранник является кратным корнем любого многочлена объема. Можно ожидать, что это верно и для объема любого нежесткого многогранника (т. е. многогранника, допускающего нетри- 
виальные бесконечно малые изгибания 1-го порядка). Обратное утверждение неверно: как было отмечено в п. 18.1, существуют метрические октаэдры Брикара 2-го рода, для которых положительный корень кратности 2 многочлена объема реализуется как объем некоторого реально существующего октаэдра, и этот октаэдр не является изгибаемым, так как все изгибаемые октаэдры имеют объем 0 (правда, мы не проверяли, является ли этот октаэдр жестким, так что вопрос об обратимости предположения для нежестких многогранников пока остается открытым).

7. Пусть квадрат объема $V_{0}^{2}$ некоторого многогранника является кратным корнем канонического многочлена объема (для многогранников рода 0 мы знаем, что это такое, а в случае рода $g>0$ предположим, что есть какое-то гипотетическое определение канонического многочлена). Вопрос - каков геометрический смысл порядка кратности корня? Здесь надо будет различать два случая: многогранник изгибаемый или неизгибаемый. Как показал студент МГУ А. Проскуряков (дипломная работа, 2005 г., не опубликовано), для изгибаемых октаэдров значение порядка кратности квадрата объема (7 для октаэдров Брикара 1-го рода и 6 - для октаэдров 2-го рода) связано с числом различных конфигураций многогранника, неналожимых друг на друга изгибанием. Может быть, на ответ влияет не факт изгибаемости, а порядок существующих бесконечно малых изгибаний высших порядков.

8. Пусть число $V_{0}^{2} \geqslant 0$ - корень многочлена объема, построенного по данной натуральной развертке многогранника. В каком случае этот корень является значением квадрата объема реально существующего многогранника, изометричного данной развертке? Как мы знаем из раздела 18, для октаэдров не всякий корень многочлена объема является объемом реально существующего октаэдра с данной метрикой.

9. Допустим, что какой-то корень $V_{0}$ канонического многочлена объема не является объемом реально существующего в $\mathbb{R}^{3}$ многогранника. Существует ли многогранник с комплексными координатами вершин, формально определенный обобщенный объем которого совпадает со значением этого корня?

10. Найти способ для более краткой записи многочлена объема (например, как-нибудь группируя слагаемые, вводя новые переменные, скажем, площади, и т. д.). В связи с этим заметим, что в 4-мерном пространстве симплекс имеет одинаковое число одномерных и двумерных граней $\left(=C_{5}^{2}=C_{5}^{3}=10\right)$. Одномерные грани - это ребра, и 4-мерный объем симплекса выражается через определитель Кэли-Менгера с использованием квадратов длин ребер. Спрашивается, можно ли выразить длины ребер через площади двумерных граней или, по крайней мере, выразить объем через площади двумерных граней? В “фольклорной" форме этот вопрос известен давно, а обсуждение похожего вопроса в [106] дает надежду на положительный ответ. Аналогичный вопрос можно поставить в общем, $n>4$, случае: в $n$-мерном симплексе в $E^{n}$ имеется одинаковое число $k$-мерных и $(n-1-k)$-мерных симплексов, в частности, одинаковое количество одномерных симплексов (ребер) и $(n-2)$-мерных симплексов. Есть ли формулы, выражающие связи между объемами соответствующих размерностей этих симплексов, в частности, можно ли выразить $n$-мерный объем симплекса через объемы его граней каких-либо размерностей выше первой? 
11. Написать компьютерную программу для нахождения многочлена объема для многогранников с малым числом вершин, реально работающую хотя бы в пределах 10 вершин. Может быть, этот вопрос не удастся решить для общего случая (с буквенным набором длин всех ребер), тогда можно работать в двух направлениях - предполагать равенство некоторых длин, так что разных длин будет меньше, чем число ребер (например, это случай многогранника Штеффена), или же предполагать, что мы знаем конкретные численные значения длин ребер (как это сделано, например, в [11; с. 18]).

12. Найти алгоритм для нахождения канонического многочлена объема, не обязательно с полиномиально растущим временем. "Наивный" алгоритм для многогранников рода 0 есть - найти какой-нибудь многочлен по первому методу доказательства теоремы 1, а затем найти нужный многочлен среди его делителей.

13. Найти формулу или многочлен для объема симплициальных многогранников данного рода $g>0$ с минимальным числом вершин. Это минимальное число $n_{\min }(g)$ определяется формулой (см. [34])

$$
\left.n_{\min }(g)=\right] \frac{1}{2}(7+\sqrt{1+48 g})[, \quad g \geqslant 0, \quad g \neq 2,
$$

где $] a[$ - ближайшее к $a$ натуральное число, не меньшее $a$ (для $g=2$ имеем исключение: $\left.n_{\min }(2)=10\right)$. В случае отсутствия диагоналей (т. е. для многогранников рода $\left.g=m(m+1) / 2, m, g \in \mathbb{N}_{0}\right)$ объем выражается через длины их ребер точной формулой (см. раздел 16). Но существуют последовательности значений $g$, для которых многогранники с минимальным числом вершин имеют неограниченно возрастающую последовательность числа диагоналей [107].

14. Охарактеризовать те нежесткие многогранники, для которых вариация объема при деформации бесконечно малого изгибания равна нулю. В работе [108] приведен алгоритм для проверки продолжаемости бесконечно малого изгибания в "настоящее" изгибание. Но в то время еще не была известна инвариантность объема при изгибании, поэтому сейчас можно рассмотреть ту же задачу уже с учетом свойства инвариантности объема. Эта задача кажется относительно нетрудной для теоретического ее решения, интересной будет ее программная реализация. Может быть, при этом исследовании окажутся полезными и идеи работ [109] и [110].

15. Можно предложить большой круг исследований по переносу всех рассмотренных задач, начиная с их постановок, на многогранники в пространствах, локально являющихся пространствами постоянной кривизны.

Несомненно, в алгебре должны быть объекты и задачи, аналогичные рассмотренным здесь геометрическим объектам и задачам. Можно надеяться, что алгебра и геометрия многоугольников и многогранников связаны так же тесно, как анализ и дифференциальная геометрия кривых и поверхностей.

В заключение пользуюсь случаем поблагодарить Л.Л. Безкоровайную, Й. Валлнера (Johannes Wallner), А. Ю. Веснина, Э. Б. Винберга, А. Д. Медных, Х. Штахеля (Hellmuth Stachel) и особенно В.А. Александрова за их внимание к моим многочисленным просьбам о помощи в поиске литературы и полезные консультации. 


\section{Список литературы}

[1] A.-M. Legendre, Eléments de géométrie, Paris, 1794.

[2] Евклид, Начала, т. 3, Пер. с греч. Д. Д. Мордухай-Болтовского, ГИТТЛ, М.-Л., 1950.

[3] A. Cauchy, "Deuxième mémoire sur les polygones et polyèdres", J. Ecole Polytechnique, 19 (1813), 87-98.

[4] P. Gario, "Il teorema di Cauchy sulle rigidita dei poliedri convessi", Archimede, 33:1-2 (1981), 53-69.

[5] А.Д. Милка, Что такое геометрия "в целом", Новое в жизни, науке, технике. Математика, кибернетика, 12, Знание, М., 1986, 31 с.

[6] A. D. Milka, "Unidentified Egyptian geometry", European J. Combin., 31:4 (2010), 1065-1071.

[7] R. Bricard, "Mémoire sur la théorie de l'octaèdre articulé", J. de Math. (5), 5:3 (1897), 113-148.

[8] H. Gluck, "Almost all simply connected closed surfaces are rigid", Geometric topology (Park City, UT, 1974), Lecture Notes in Math., 438, Springer, Berlin, 1975, 225-239; pyc. пер.: Г. Глюк, "Почти все односвязные замкнутые поверхности неизгибаемы", Исследования по метрической теории поверхностей, Математика: новое в зарубежной науке, 18, Мир, М., 1980, 148-163.

[9] R. Connelly, "An immersed polyhedral surface which flexes", Indiana Univ. Math. J., 25:10 (1976), 965-972.

[10] R. Connelly, "A counterexample to the rigidity conjecture for polyhedra", Inst. Hautes Études Sci. Publ. Math., 47 (1977), 333-338.

[11] И.Х. Сабитов, Объемы многогранников, МЦНМО, М., 2002, 32 с., http://www. mccme.ru/free-books/mmmf-lectures/book.21.pdf.

[12] N.H. Kuiper, "Sphères polyèdriques flexibles dans $E^{3}$, d'après Robert Connelly", Séminaire Bourbaki, 30ème année, Exp. № 514 (1977/78), Lecture Notes in Math., 710, Springer, Berlin, 1978, 147-168; pyc. пер.: Н. Кёйпер, "Изгибаемые полиэдральные сферы в $E^{3}$, по Роберту Коннелли", Исследования по метрической теории поверхностей, Математика: новое в зарубежной науке, 18, Мир, М., $1980,210-227$.

[13] R. Connelly, "Conjectures and open questions in rigidity", Proceedings of the International Congress of Mathematicians (Helsinki, 1978), Acad. Sci. Fennica, Helsinki, 1980, 407-414; рус. пер.: Р. Коннелли, "Некоторые предположения и нерешенные вопросы в теории изгибаний", Исследования по метрической теории поверхностей, Математика: новое в зарубежной науке, 18, Мир, М., 1980, 228-238.

[14] J.-M. Schlenker, "La conjecture des soufflets (d'après I. Sabitov)", Séminaire N. Bourbaki, 2002-2003 (2002), Exp. 909-923; Astérisque, 294, Soc. Math. France, Paris, 2004, 77-95.

[15] I. Pak, Lectures on discrete and polyhedral geometry, Preprint, 2008.

[16] В. А. Александров, "Замечания к гипотезе Сабитова о стационарности объема при бесконечно малом изгибании поверхности", Сиб. матем. журн., 30:5 (1989), 16-24; англ. пер.: V. A. Aleksandrov, "Remarks on Sabitov's conjecture on volume rigidity with infinitesimal bending of a surface", Siberian Math. J., 30:5 (1989), 678-684.

[17] В. А. Александров, "Новый пример изгибаемого многогранника", Сиб. матем. журн., 36:6 (1995), 1215-1224; англ. пер.: V. A. Aleksandrov, "A new example of a flexible polyhedron", Siberian Math. J., 36:6 (1995), 1049-1057.

[18] C. Schwabe, "Flexing polyhedra", Symmetry. Cult. Sci., 3:2 (1992), 168-169, 213-221. 
[19] И.Х. Сабитов, "Объем многогранника как функция его метрики", Фундам. и прикл. матем., 2:4 (1996), 1235-1246.

[20] И. Х. Сабитов, "К проблеме об инвариантности объема изгибаемого многогранника", УМH, 50:2 (1995), 223-224; англ. пер.: I. Kh. Sabitov, "On the problem of invariance of the volume of a flexible polyhedron", Russian Math. Surveys, 50:2 (1995), 451-452.

[21] И.Х. Сабитов, “Обобщенная формула Герона-Тарталья и некоторые ее следствия", Матем. сб., 189:10 (1998), 105-134; англ. пер.: I. Kh. Sabitov, "A generalized Heron-Tartaglia formula and some of its consequences", Sb. Math., 189:10 (1998), 1533-1561.

[22] I. Kh. Sabitov, "The volume as a metric invariant of polyhedra", Discrete Comput. Geom., 20:4 (1998), 405-425.

[23] R. Connelly, I. Sabitov, A. Walz, "The bellows conjecture", Beiträge Algebra Geom., 38:1 (1997), 1-10, http://www.emis.de/journals/BAG/vol.38/no.1/1.html.

[24] I. Kh. Sabitov, "On some recent results in the metric theory of polyhedra", III International Conference in Stochastic Geometry, Convex Bodies and Empirical Measures, Part II (Mazara del Vallo, 1999), Rend. Circ. Mat. Palermo (2), Suppl. No. 65, part II, 2000, 167-177.

[25] I. Sabitov, "Solution of polyhedra", Bull. Braz. Math. Soc., 35:2 (2004), 199-210.

[26] И. Х. Сабитов, "Решение циклических многоугольников", Матем. просвещение, 2010, № 14, 83-106, http://www.mccme.ru/free-books/matpros/mpe.pdf.

[27] V.A. Alexandrov, I. Kh. Sabitov, H. Stachel, "Rigidity and related topics in geometry", European J. Combin., 31:4 (2010), 1035-1036.

[28] И. Х. Сабитов, “Локальная теория изгибаний поверхностей”, Итоги науки и техники. Соврем. проблемы матем. Фундам. напр., 48, ВИНИТИ, М., 1989, 196-270.

[29] И. Иванова-Каратопраклиева, И. Х. Сабитов, "Изгибание поверхностей. II", Итоги науки и техники. Соврем. матем. и ее прил. Темат. обзоры, 8, ВИНИТИ, M., 1995, 108-167; англ. пер.: I. Ivanova-Karatopraklieva, I. Kh. Sabitov, "Bending of surfaces. Part II", J. Math. Sci., 74:3 (1995), 997-1043.

[30] A. Császár, "A polyhedron without diagonals", Acta Univ. Szeged. Sect. Sci. Math., 13 (1949), 140-142.

[31] А. В. Бушмелев, С. А. Лавренченко, "Многогранники-торы”, Квант, 1985, № 2, 3-5, http://kvant.mirror1.mccme.ru/1985/02/mnogogranniki-tory.htm.

[32] S. Lawrencenko, S. Negami, I. Kh. Sabitov, "A simpler construction of volume polynomials for a polyhedron", Beiträge Algebra Geom., 43:1 (2002), 261-273.

[33] L. M. Blumental, Theory and applications of distance geometry, 2nd ed., Chelsea, New York, 1970, xi+347 pp.

[34] M. Jungerman, G. Ringel, "Minimal triangulations on orientable surfaces", Acta Math., 145:1-2 (1980), 121-154.

[35] S. Lang, Introduction to algebraic geometry, 3rd ed., Addison-Wesley, Reading, MA, 1972, xi+260 pp.

[36] Ю.И. Мерзляков, Рационалъные группы, 2-е изд., Наука, М., 1987, 448 с.

[37] А. Д. Александров, Выпуклые многогранники, Гостехиздат, М.-Л., 1950, 428 с.; А. Д. Александров, Избранные труды, т. 2: Выпуклые многогранники, Наука, Новосибирск, 2007, 491 с.; англ. пер.: А. D. Aleksandrov, Convex polyhedra, Springer Monogr. Math., Springer, Berlin, 2005, ISBN: 3-540-23158-7, xii+539 pp.

[38] Ю. Д. Бураго, В. А. Залгаллер, "Изометрические кусочно-линейные погружения двумерных многообразий с полиэдральной метрикой в $\mathbb{R}^{3 "}$, Алгебра и анализ, 7:3 (1995), 76-95; англ. пер.: Yu. D. Burago, V. A. Zalgaller, "Isometric piecewise-linear embeddings of two-dimensional manifolds with a polyhedral metric into $\mathbb{R}^{3 "}$, St. Petersburg Math. J., 7:3 (1996), 369-385. 
[39] А. В. Астрелин, И. Х. Сабитов, "Канонический многочлен для объема многогранника", УМH, 54:2 (1999), 165-166; англ. пер.: A. V. Astrelin, I. Kh. Sabitov, "A canonical polynomial for the volume of a polyhedron", Russian Math. Surveys, $\mathbf{5 4 : 2}$ (1999), 430-431.

[40] I. Kh. Sabitov, "On polyhedra with calculable diagonals", IV International Conference in Stochastic Geometry, Convex Bodies, Empirical Measures \& Applications to Engineering Science, v. II (Tropea, 2001), Rend. Circ. Mat. Palermo (2), Suppl. No. 70, part II, 2002, 289-294.

[41] О. В. Павлова, "Объем подвески как функция длин ее ребер", УМH, 50:4 (1995), 165-166; англ. пер.: O. V. Pavlova, "The volume of a pendant as a function of the lengths of its edges", Russian Math. Surveys, 50:4 (1995), 826-827.

[42] А. В. Астрелин, И. Х. Сабитов, "Минимальный по степени многочлен для определения объема октаэдра по его метрике", УМН, 50:5 (1995), 245-246; англ. пер.: A. V. Astrelin, I. Kh. Sabitov, "A minimal-degree polynomial for determining the volume of an octahedron from its metric", Russian Math. Surveys, 50:5 (1995), 1085-1087.

[43] Р. В. Галиулин, С.Н. Михалёв, И. Х. Сабитов, "Некоторые приложения формулы для объема октаэдра", Матем. заметки, 76:1 (2004), 27-43; англ. пер.: R. V. Galiulin, S. N. Mikhalev, I. Kh. Sabitov, "Some applications of the formula for the volume of an octahedron", Math. Notes, 76:1-2 (2004), 25-40.

[44] A. I. Balabin, R. O. Sack, "Thermodynamics of (Zn,Fe)S sphalerite. A CVM approach with large basis clusters", Mineralogical Magazine, 64:5 (2000), 923-943.

[45] С. Н. Михалёв, "Изометрические реализации октаэдров Брикара 1-го и 2-го типа с известными значениями объема", Фундам. и прикл. матем., 8:3 (2002), $755-768$.

[46] И.Х. Сабитов, “Алгоритмическое решение проблемы изометрической реализации двумерных многогранных метрик", Изв. РАН. Сер. матем., 66:2 (2002), 159-172; англ. пер.: I. Kh. Sabitov, "Algorithmic solution of the problem of isometric realization for two-dimensional polyhedral metrics", Izv. Math., 66:2 (2002), 377-391.

[47] J. Graver, "Rigidity of triangulated surfaces", Colloquium during the Special Semester on Structural Rigidity, Centre de Recherches Mathématiques, Univ. de Monréal, 1987.

[48] W. Whiteley, Infinitesimal rigid polyhedra III: triangulated tori, Preprint, Champlain Regional College, St. Lambert, QC, 1985.

[49] A. Fogelsanger, The generic rigidity of minimal cycles, Preprint, Cornell Univ., 1987, 60 p.; A. Fogelsanger, The generic rigidity of minimal cycles, Ph. D. Thesis, Cornell Univ., 1988, 68 pp.

[50] И. Х. Сабитов, “Алгоритмическая проверка изгибаемости подвесок”, Укр. геом. сб., 30 (1987), 109-112.

[51] И. Г. Максимов, И. Х. Сабитов, "О понятии комбинаторной $p$-параметричности многогранников", Сиб. матем. журн., 43:4 (2002), 823-839; англ. пер.: I. G. Maksimov, I. Kh. Sabitov, "On the notion of the combinatorial p-parameter property for polyhedra", Siberian Math. J., 43:4 (2002), 661-673.

[52] И. Г. Максимов, "Исследование изгибаний алгоритмически 1-параметрических многогранников и описание одного множества неизгибаемых вложенных многогранников", Сиб. матем. журн., 51:6 (2010), 1367-1378; англ. пер.: I. G. Maksimov, "Study of flexible algorithmically 1-parametric polyhedra and description of a set of rigid embedded polyhedra", Siberian Math. J., 51:6 (2010), 1081-1090.

[53] И.Г. Максимов, Описание комбинаторного строения алгоритмически 1-параметрических многогранников типа сферы, Препринт, 2011. 
[54] M. Fedorchuk, I. Pak, "Rigidity and polynomial invariants of convex polytopes", Duke Math. J., 129:2 (2005), 371-404.

[55] Э. Б. Винберг, Курс алгебры, Факториал, М., 2001; англ. пер.: Е. В. Vinberg, A course in algebra, Grad. Stud. Math., 56, Amer. Math. Soc., Providence, 2003.

[56] С. А. Лавренченко, "Неприводимые триангуляции тора", Укр. геом. сб., 1987, № 30, 52-62; англ. пер.: S. A. Lavrenchenko, "Irreducible triangulations of a torus", J. Soviet Math., 51:5 (1990), 2537-2543.

[57] K. G. Ch. von Staudt, "Über die Inhalte der Polygone und Polyeder", J. Reine Angew. Math. [Crelle's journal], 24 (1842), 252-256.

[58] J. J. Sylvester, "On Staudt's theorems concerning the contents of polygons and polyhedrons, with a note on a new and resembling class of theorems", Philosophical Magazine, IV (1852), 335-345; перепеч. в кн.: J. J. Sylvester, The collected mathematical papers, v. 1, Cambridge Univ. Press, Cambridge, 1904, 382-391.

[59] A. I. Bobenko, I. Izmestiev, "Alexandrov's theorem, weighted Delauney triangulations and mixed volumes", Ann. Inst. Fourier (Grenoble), 58:2 (2008), 447-505.

[60] A. I. Bobenko, I. Izmestiev, "A variational proof of Alexandrov's convex cap theorem", Discr. Comput. Geom., 40:4 (2008), 561-585.

[61] С.Н. Михалёв, "Об одном методе решения задачи изометрической реализации разверток", Фундам. и прикл. матем., 12:1 (2006), 167-203; англ. пер.: S.N. Mikhalev, "A method for solving the problem of isometric realization of developments", J. Math. Sci. (N. Y.), 149:1 (2008), 971-995.

[62] H. Whitney, "A theorem on graphs", Ann. of Math. (2), 32:2 (1931), 378-390.

[63] I. Kh. Sabitov, "On a property of volume polynomials for flexing polyhedra", $M a-$ ломерная топология и комбинаторная теория групп (Челябинск, 1999), Киев, $2000,315-318$.

[64] R. Connelly, "The rigidity of polyhedral surfaces", Math. Mag., 52:5 (1979), 275-283.

[65] В. Г. Болтянский, Третъя проблема Гильберта, Наука, М., 1977, 207 с.; англ. пер.: V. G. Boltianskii, Hilbert's third problem, Scripta Ser. in Math., Winston, Washington, WA; Halsted Press, New York-Toronto-London, 1978, 228 pp.

[66] C.-H. Sah, Hilbert's third problem: scissors congruence, Res. Notes in Math., 33, Pitman, Boston, MA, 1979, ISBN: 0-273-08426-7, vi+188 pp.

[67] P. Cartier, "Décomposition des polyèdres: le point sur le troisième problème de Hilbert", Astérisque, 133-134 (1986), 261-286.

[68] J.L. Dupont, Scissors congruences, group homology and characteristic classes, Nankai Tracts Math., 1, World Scientific, River Edge, NJ, 2001, viii+168 pp.

[69] V. Alexandrov, The Dehn invariants of the Bricard octahedra, arXiv: 0901.2989.

[70] V. Alexandrov, R. Connelly, "Flexible suspensions with a hexagonal equation", Illinois J. Math. (to appear).

[71] R. Alexander, "Lipschitzian mappings and total mean curvature of polyhedral surfaces. I", Trans. Amer. Math. Soc., 288 (1985), 661-678.

[72] V. Alexandrov, "Algebra versus analysis in the theory of flexible polyhedra", Aequationes Math., 79:3 (2010), 229-235.

[73] V. Alexandrov, "An example of a flexible polyhedron with nonconstant volume in the spherical space", Beiträge Algebra Geom., 38:1 (1997), 11-18.

[74] Д. А. Слуцкий, "Нежесткий многогранник с ненулевой вариацией объема в пространстве Лобачевского", Сиб. матем. журн., 52:1 (2011), 167-176; англ. пер.: D. A. Slutskiy, "An infinitesimally nonrigid polyhedron with nonstationary volume in the Lobachevsky 3-space", Siberian Math. J., 52:1 (2011), 131-138; arXiv: 1002.3884 .

[75] Н. В. Абросимов, М. Годой-Молина, А.Д. Медных, "Об объеме сферического октаэдра с симметриями", Соврем. матем. и ее прил., 60:1 (2008), 1-12; англ. 
пер.: N.V. Abrosimov, M. Godoi-Molina, A.D. Mednykh, "On the volume of a spherical octahedron with symmetries", J. Math. Sci. (N. Y.), 161:1 (2009), 1-10.

[76] H. Busemann, P. J. Kelly, Projective geometry and projective metrics, Academic Press, New York, 1953, viii+332 pp.; pyc. пер.: Г. Буземан, П. Келли, Проективная геометрия и проективнье метрики, ИЛ, М., 1957, 410 с.

[77] В. В. Прасолов, Геометрия Лобачевского, МЦНМО, М., 2004, http://www. mccme.ru/free-books/prasolov/glob.pdf.

[78] Д.А. Деревнин, А.Д. Медных, “О формуле объема гиперболического тетраэдра", УМН, 60:2 (2005), 159-160; англ. пер.: D. A. Derevnin, A. D. Mednykh, "A formula for the volume of a hyperbolic tetrahedron", Russian Math. Surveys, 60:2 (2005), 346-348.

[79] Yunhi Cho, Hyuk Kim, "On the volume formula for hyperbolic tetrahedra", Discrete Comput. Geom., 22:3 (1999), 347-366.

[80] J. Murakami, M. Yano, "On the volume of a hyperbolic and spherical tetrahedron", Comm. Anal. Geom., 13:2 (2005), 379-400.

[81] A.H. Ushijima, "A volume formula for generalised hyperbolic tetrahedra", NonEuclidean geometries, Math. Appl. (N. Y.), 581, Springer, New York, 2006, 249-265.

[82] А. Ю. Веснин, "Объемы трехмерных гиперболических многообразий Лебелля", Матем. заметки, 64:1 (1998), 17-23; англ. пер.: А. Yu. Vesnin, "Volumes of hyperbolic Löbell 3-manifolds", Math. Notes, 64:1 (1998), 15-19.

[83] А. Ю. Веснин, А. Д. Медных, "Гиперболические объемы многообразий Фибоначчи", Сиб. матем. журн., 36:2 (1995), 266-277; англ. пер.: А. Yu. Vesnin, A.D. Mednykh, "Hyperbolic volumes of Fibonacci manifolds", Siberian Math. J., 36:2 (1995), 235-245.

[84] A. Vesnin, On volumes of some hyperbolic 3-manifolds, Lecture Notes Ser., 30, Seoul National Univ. Global Analysis Research Center, Seoul, 1996, iv+132 pp.

[85] А. Д. Медных, “Объемы многогранников в пространстве Лобачевского", Труды Математического иентра им. Н. И. Лобачевского, 39 (2009), 265-267.

[86] Э.Б. Винберг, О.В. Шварцман, “Дискретные группы движений пространств постоянной кривизны”, Геометрия - 2, Итоги науки и техники. Соврем. проблемы матем. Фундам. напр., 29, ВИНИТИ, М., 1988, 147-259; англ. пер.: Eh. B. Vinberg, O. V. Shvartsman, "Discrete groups of motions of spaces of constant curvature", Geometry. II: Spaces of constant curvature, Encycl. Math. Sci., 29, Springer, Berlin, 1993, 139-248.

[87] H. Stachel, "Flexible octahedra in the hyperbolic space", Non-Euclidean geometries, Math. Appl. (N. Y.), 581, Springer, New York, 2006, 209-225.

[88] Н. В. Абросимов, "К решению проблемы Зейделя об объемах гиперболических тетраэдров", Сиб. электрон. матем. изв., 6 (2009), 211-218.

[89] Н. В. Абросимов, "Проблема Зейделя об объеме неевклидова тетраэдра", Докл. PAH, 435:1 (2010), 7-10.

[90] A. Mednykh, A. Rasskazov, "Volumes and degeneration of cone-structures on the figure-eight knot", Tokyo J. Math., 29:2 (2006), 445-464.

[91] А.А. Колпаков, А.Д. Медных, "Сферические структуры на торических узлах и зацеплениях", Сиб. матем. журн., 50:5 (2009), 1083-1096; англ. пер.: A. A. Kolpakov, A. D. Mednykh, "Spherical structures on torus knots and links", Siberian Math. J., 50:5 (2009), 856-866.

[92] R. N. Shmatkov, "Properties of Euclidean Whitehead link cone-manifolds", Siberian Adv. Math., 13:1 (2003), 55-86.

[93] H. Stacherl, "Flexible cross-polytopes in the Euclidean 4-space", J. Geom. Graph., 4:2 (2000), 159-167, http://www.heldermann.de/JGG/jggabs04.htm\#art14.

[94] C.S. Borcea, "Infinitesimally flexible skeleta of cross-polytopes and second-hypersimplices", J. Geom. Graph., 12:1 (2008), 1-10. 
[95] M. M. Lin, M. T. Chu, "On the nonnegative rank of Euclidean distance matrices", Linear Algebra Appl., 433:3 (2010), 681-689.

[96] I. Z. Emiris, T. G. Nikitopoulos, "Molecular conformation search by distance matrix perturbations", J. Math. Chem., 37:3 (2004), 233-253.

[97] И. Х. Сабитов, "Новые классы неизгибаемых многогранников”, Всесоюзнал конферениия по геометрии и анализу, ИМ СО АН СССР, Новосибирск, 1989, 72.

[98] Н. О. Ермилов, "Восстановление тетраэдров по заданному набору длин ребер", Междунар. конф. "Метрическая геометрия поверхностей и многогранников", посв. 100-летию Н. В. Ефимова (М., 2010), Макс Пресс, М., 2011, 25-26.

[99] Н. О. Ермилов, "Восстановление тетраэдра по заданному набору длин ребер", Матем. заметки (в печати).

[100] O.R. Musin, "Sets with few distances", Междунар. конф. "Метрическал геометрия поверхностей и многогранников", посв. 100-летию Н. В. Ефимова (М., 2010), Макс Пресс, М., 2011, 77-78.

[101] C. Borcea, I. Streinu, "The number of embeddings of minimally rigid graphs", Discrete Comput. Geom., 31:2 (2004), 287-303.

[102] H. Whitney, "Congruent graphs and the connectivity of graphs", Amer. J. Math., 54:1 (1932), 150-168.

[103] М. А. Бродский, В.Н. Страхов, "В классе однородных многогранников, гомеоморфных шару, решение обратной задачи Ньютонова потенциала единственно", Докл. АН ССCP, 292:6 (1987), 1337-1340; англ. пер.: M. A. Brodskii, V.N. Strakhov, "In the class of homogeneous polyhedra homeomorphic to a ball, the solution of the inverse Newtonian potential problem is unique", Soviet Phys. Dokl., 32:2 (1987), 111-113.

[104] S. Lavrenchenko, "An infinite set of torus triangulations of connectivity 5 whose graphs are not uniquely embeddable in the torus", Discrete Math., 66:3 (1987), 299-301.

[105] С. А. Лавренченко, "О числе треугольных укладок вершинно-помеченного графа на тор", Укр. геом. сб., 31 (1988), 76-90; англ. пер.: S. A. Lavrenchenko, "The number of triangular packings of a vertically labeled graph on a torus", J. Soviet Math., 54:1 (1991), 719-728.

[106] P. McMullen, "Simplices with equiareal faces", Discrete Comput. Geom., 24:2-3 (2000), 397-411.

[107] О.Г. Давыдов, Э.Р. Розендорн, “Число диагоналей у многогранников рода $g$ с треугольными гранями и минимальным числом вершин", Инвариантные методы исследования на многообразиях структур геометрии, анализа и математической физики (конф., посв. 90-летию Г. Ф. Лаптева). Ч. 1, Изд-во мех.-мат. ф-та МГУ, М., 2001, 59-69.

[108] И.Х. Сабитов, "Об одном алгоритме проверки изгибаемости многогранника", Вестн. Моск. ун-та. Сер. 1. Матем., мех., 1994, № 2, 56-61; англ. пер.: I. Kh. Sabitov, "On an algorithm for testing the bendability of a polyhedron", Moscow Univ. Math. Bull., 49:2 (1994), 50-54.

[109] V. Alexandrov, "Sufficient conditions for the extendibility of a nonconstant $n$-th order flex of polyhedra", Beiträge Algebra Geom., 39:2 (1998), 367-378.

[110] V. Alexandrov, "Implicit function theorem for systems of polynomial equations with vanishing Jacobian and its application to flexible polyhedra and framworks", Monatsh. Math., 132:4 (2001), 269-288.

И. Х. Сабитов (I. Kh. Sabitov)

Московский государственный университет

им. М. В. Ломоносова

E-mail: isabitov@mail.ru
Поступила в редакцию 08.07.2010 